\title{
Linear viscoelasticity and flow of self-assembled vitrimers: the case of a polyethylene/dioxaborolane system
}

\author{
Ralm G. Ricarte, ${ }^{\dagger *}$ François Tournilhac, ${ }^{\dagger}$ Michel Cloître, ${ }^{\dagger}$ and Ludwik Leibler ${ }^{*}$
}

${ }^{\dagger}$ Molecular, Macromolecular Chemistry, and Materials, ESPCI Paris, CNRS, PSL Research University, 75005 Paris, France and $\$$ Gulliver, ESPCI Paris, CNRS, PSL Research University, 10 Rue Vauquelin, 75005 Paris, France

Email: ralm.ricarte@espci.fr and ludwik.leibler@espci.fr 


\section{Abstract}

For vitrimer systems obtained by dynamic cross-linking of polymer chains, incompatibility effects between the cross-links and polymer backbone can lead to microphase separation, resulting in a network made of cross-linked aggregates. Additionally, when there is a wide distribution of the number of cross-links per chain, macrophase separation can occur, mostly due to entropic effects. Here, we investigate the linear viscoelasticity and flow of a polyethylene (PE) vitrimer that has cross-linkable dioxaborolane maleimide grafts, which self-assemble into a hierarchical nanostructure. To elucidate the role of self-assembly, we first studied dioxaborolane graft functionalized PE that was non-cross-linked. It had a terminal relaxation time that was orders of magnitude larger than both neat PE and partially peroxide cross-linked PE. When dioxaborolane cross-linker was added to form the vitrimer, the resulting material could not achieve terminal relaxation within $8 \mathrm{~h}$. The soluble and insoluble portions of the PE vitrimer were then isolated and characterized. The soluble portion, which was graft-poor, expressed similar flow behavior as neat PE, while the insoluble portion - which was a graft-rich network of cross-linked aggregates relaxed very little over $8 \mathrm{~h}$. When the insoluble and soluble portions were blended, the rheological behavior of the original vitrimer was basically recovered, showing that the soluble portion acts as a lubricant. When the insoluble portion was blended with neat PE, the material relaxed much more stress, but still did not reach steady-state flow within $8 \mathrm{~h}$. When high stresses were applied, however, PE vitrimer flowed. Nonlinear rheology experiments revealed melt fracture at high strains and suggested that flow is enabled by rapid healing, which follows fracture events. The presence of macroscopic phase separation facilitated flow.

Keywords: vitrimers, self-assembly, rheological properties 


\section{Introduction}

Vitrimers are covalent networks that engage in thermoactivated associative exchange reactions. ${ }^{1,2,3}$ Under cooling, they undergo a reversible topology freezing transition, analogous to the glass transition of amorphous silica. In contrast to dissociative cross-links, the associative cross-links of vitrimers preserve network connectivity at all times and temperatures below degradation conditions, yet still enable the network topology to fluctuate. ${ }^{1,2,3}$ The consequences of the topology freezing transition on materials properties were investigated for epoxy/acid systems that underwent catalyst-driven transesterification. ${ }^{1}$ Since then, the literature has focused on altering the exchange reaction (either catalytically controlled or catalyst-free) and adapting chemistries to different polymer backbones. $2,3,4,5,6,7,8,9,10,11,12,13,14,15,16,17,18,19,20,21,22,23,24,25,26,27$ Other studies explored the properties of vitrimer composites and the addition of nondynamic cross-links to the network. ${ }^{14,28,29,30,31,32,33,34,35}$ These research efforts established that modifying vitrimer chemistry provides a pathway for tuning mechanical properties, ${ }^{36,37,38}$ stress relaxation, ${ }^{39,40,41,42}$ shape memory, ${ }^{43,44,45}$ and the ability to self-heal and adhere to other materials. ${ }^{21,46}$ Due to this versatility, vitrimers are now being tested for a variety of high-end applications. ${ }^{47,48,49,50,51}$ Recent reviews have summarized this progress in developing vitrimer chemistry, materials, and applications. $^{52,53,54,55}$

The dynamic characteristics of vitrimers are often enabled by incorporating functional groups into homopolymer networks. The polarizability of the functional groups can be much different from that of the network monomers. Consequently, incompatibility effects may lead to network organization on mesoscopic length scales, greatly influencing dynamics and properties. Ricarte et al. showed that polyethylene/dioxaborolane vitrimers self-assemble into nanostructures. ${ }^{56}$ Chen et al. further observed microphase separation in molten polybutadiene and polydimethylsiloxane vitrimers with hydroxyurethane cross-links. ${ }^{57}$ In this work, we show that self-assembly and phase separation have a significant impact on the rheology and, consequently, processability of vitrimers. 
Studies within the past decade have focused on the dynamics of homogeneous (i.e., unstructured) vitrimers. For the epoxy/acid systems that were initially investigated by Montarnal et al., the stress relaxation behavior was studied at temperatures well above the glass transition temperature, where the transesterification exchange reaction was relatively slow compared to the network strand relaxation. Under these conditions, stress relaxation could be described by a simple Maxwell model, while the terminal relaxation time exhibited Arrhenius-like behavior. ${ }^{1}$ Subsequent work by Capelot et al. demonstrated that both the catalyst type and concentration influence the relaxation and self-healing kinetics. ${ }^{2,3,58}$ Following these initial investigations, several groups employed constitutive equations to describe vitrimer rheology and, in particular, plastic flow at intermediate time scales. ${ }^{34,35,59,60,61,62,63,64,65,66,67}$ The full and simplified theories developed by Terentjev and coworkers can even describe the stress relaxation of vitrimers bearing both exchangeable and nondynamic cross-links. ${ }^{34,35,68,69}$ Complementing the continuum model approaches, Sciortino et al. used molecular dynamics simulations to study vitrimer relaxations at the microscale. From these coarse-grained models, they posited that homogeneous vitrimers undergo two modes of relaxation: (I) a damped elastic motion and (II) reshuffling of the crosslinks within the network. ${ }^{70}$ Slow cross-link exchange kinetics lead to large viscosity, ${ }^{71}$ while topological defects (such as loops) accelerate network stress relaxation. ${ }^{72}$

For vitrimer systems that are close to the glass transition temperature, are weakly crosslinked, or have cross-links that can exchange quickly, the dynamics are controlled by both network topology fluctuations and inter-chain friction. In these situations, the vitrimer exhibits rheological behavior that is more complicated than the simple Maxwell model. For example, Snijkers et al. observed that the dynamic moduli of epoxy vitrimers - as determined by small-amplitude oscillatory shear - express multiple plateaus and peaks. ${ }^{73}$ These features are reminiscent of rheological behavior of associating polymers, which have pendant groups that act as dynamic cross-links (i.e., "stickers"). Experimentally, the elastic modulus plateaus and viscous modulus peak positions are influenced by the sticker concentration and molecular weight between 
stickers. ${ }^{74,75,76}$ These structure-dynamics relationships are predicted by sticky Rouse and sticky reptation theories. ${ }^{77,78}$

While the dynamics of homogeneous vitrimers are already intricate, the presence of heterogeneities in the system should add even more complexity. Generally, macro- and microphase separated polymer systems exhibit enthalpic barriers that slow down relaxation. In the case of immiscible polymer blends that form droplets dispersed within a matrix, application of a step strain will deform or break the droplets. The viscoelasticity of the blend reflects the interfacial tension between the two phases and the relaxation of the matrix, interface, and deformed droplet shape. ${ }^{79,80,81,82,83}$ Ionomers possess a small number of ionic groups that form clusters within the matrix of the polymer backbone. This aggregation hinders the Rouse and reptation modes of the polymer chains, delaying terminal relaxation. ${ }^{75,84,85}$ The rheology of microphase separated block polymers emanates from relaxation processes that occur at several different length scales $-e . g$, chain exchange, diffusion of chains parallel to the domain interfaces, and dissipation of lattice defects. ${ }^{86,87,88,89,90}$ For solutions of associating polymers that form large aggregates, theories have been developed to describe the various stress relaxation mechanisms of the system. ${ }^{91,92,93}$

The goal of this work is to investigate how nanostructured vitrimer materials relax and flow. The model vitrimer in this study was composed of polyethylene (PE) and dioxaborolane cross-links (see Scheme 1). Following methods introduced previously, ${ }^{21,56}$ PE vitrimer was prepared by radically grafting dioxaborolane maleimide onto the polymer backbone, followed by cross-linking via the addition of bis-dioxaborolane. The cross-links rearrange by engaging in a dioxaborolane metathesis exchange reaction. ${ }^{15,21,56}$ Interactions among the polymer backbone, dioxaborolane grafts, and cross-links induce strong incompatibility effects, causing PE vitrimer to adopt hierarchical meso- and nanostructures (Figure 1). Essentially, PE vitrimer may be described as a phase separated blend with two distinct macrophases. The insoluble phase contains a high concentration of cross-linked grafts which self-assemble into a network of aggregates (Figure 2). The soluble phase, on the other hand, possesses a small amount of grafts. ${ }^{56}$ To understand the rheology of PE vitrimer, it is necessary not only to characterize the isolated insoluble and soluble 
portions, but also to investigate blended samples to gain insight into how these macrophases interact with one another.

Scheme 1. Chemical structures of polyethylene (PE), graft-functionalized PE (PE-g), and PE vitrimer (PE-v). For illustration purposes, the PE$v$ structure includes cross-linked grafts, a pendant graft, and a pendant cross-linker.
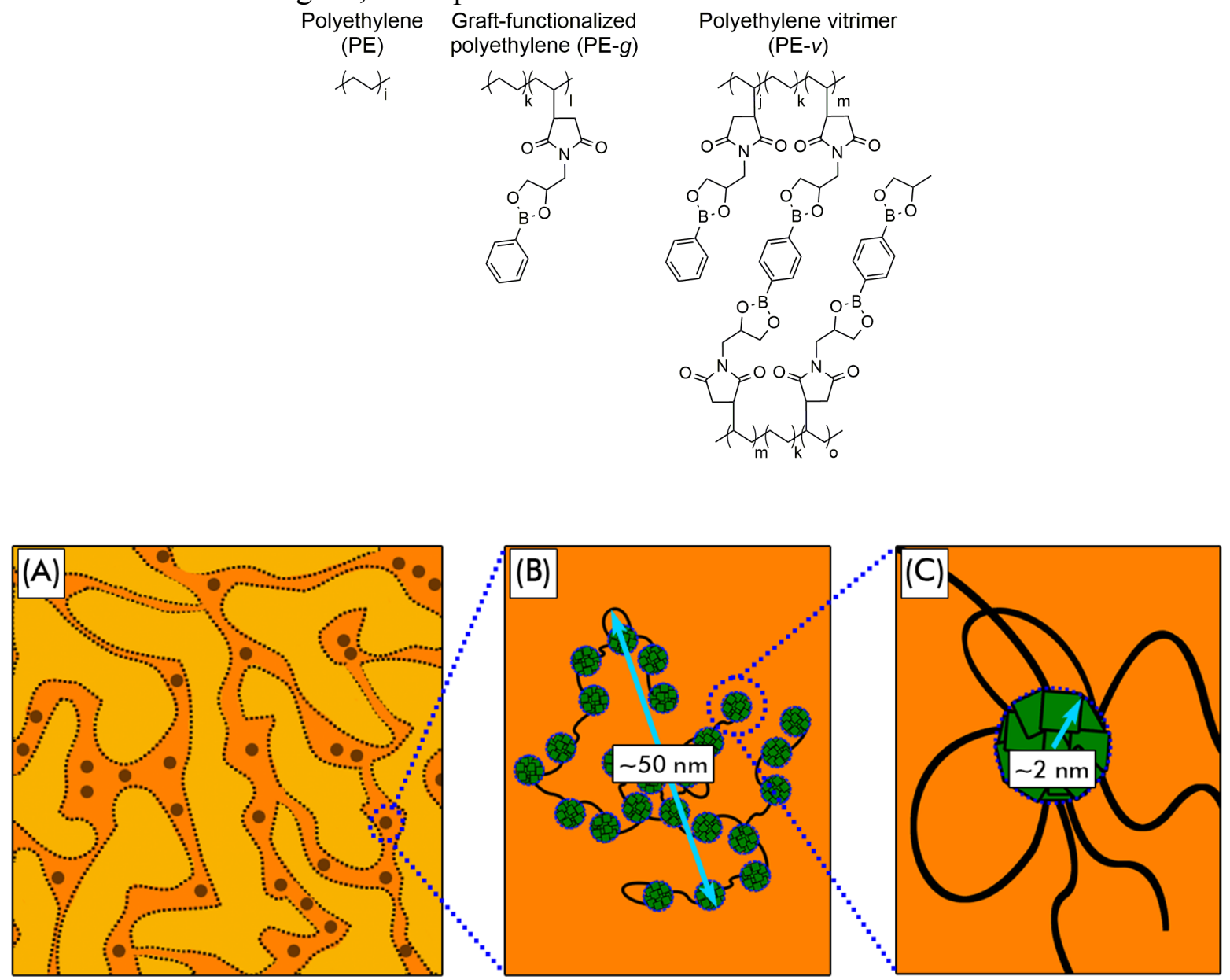

Figure 1. Schematic of the (A) mesostructure, (B) fractal structure, and (C) aggregate structure for PE vitrimer, based on study in ref. 56. In (A), the orange domain is graft-rich, the yellow domain is graft-poor, and the dashed line represents the interface. The dark circles are fractals. In (B), aggregates, PE chain loops, and PE chain bridges are shown. In (C), a single graft-rich aggregate, PE chain loops, bridges, and dangling ends are shown. 

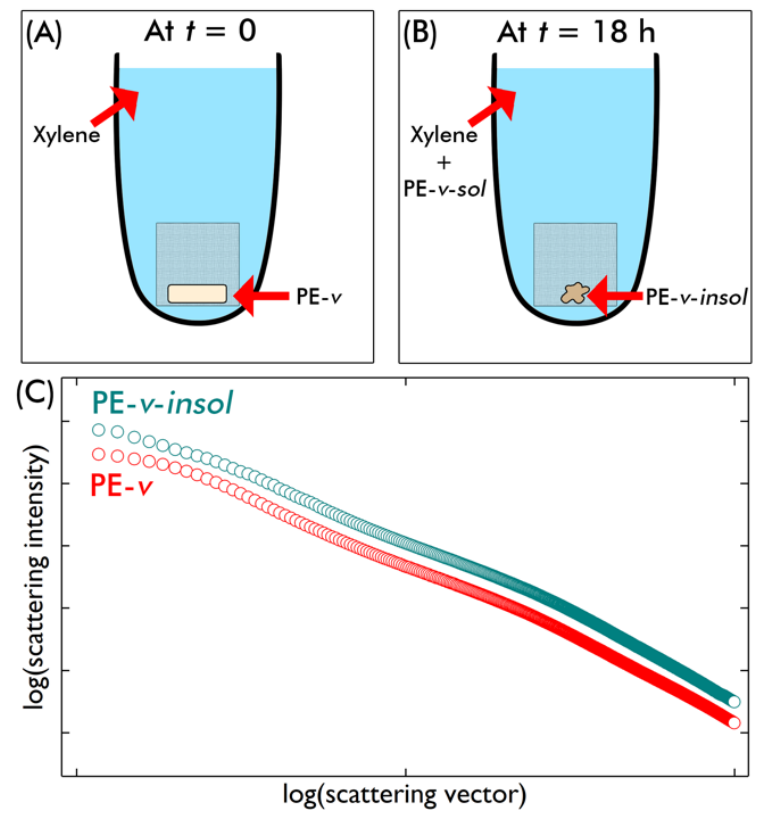

Figure 2. Schematic of $\mathrm{PE}$ vitrimer $(\mathrm{PE}-v)$ submerged in xylene at $140^{\circ} \mathrm{C}:(\mathrm{A}) t=0$ and (B) $t=18 \mathrm{~h}$. Within the $18 \mathrm{~h}$, a portion of the material dissolves (PE- $v$-sol), while the rest remains insoluble (PE-v-insol). (C) Representative small-angle X-ray scattering patterns of molten PE- $v$ and PE- $v$-insol, which confirm the samples are nanostructured. These curves are the same data seen in Figure $8 \mathrm{C}$ of ref. 56.

For vitrimers that self-assemble, associative cross-linking and macro/microphase separation likely influence the dynamics over a wide range of time scales. Therefore, multiple rheological experiments must be employed for characterization. Stress relaxation is the most commonly used tool in the vitrimer literature, but it is not suitable for systems whose stress decays by more than three decades. ${ }^{94}$ Creep experiments, in which a constant stress is applied and strain is measured, are useful for evaluating relaxation at long time scales. The technique also provides an explicit method for determining if a material fully relaxes. If the increase of the strain becomes linearly proportional to time, the material has achieved terminal relaxation (also known as steady- 
state flow). ${ }^{95}$ Small-amplitude oscillatory shear (SAOS), in which the sample is deformed sinusoidally, is appropriate for evaluating relaxation processes that occur at short time scales. ${ }^{94}$ While the aforementioned techniques probe linear viscoelasticity, ultimately, the processability of a vitrimer will mostly depend on its nonlinear behavior. For most practical processing situations, a vitrimer would be exposed to very high shear stresses and strains. ${ }^{94}$

In this case study, the linear viscoelasticity of a PE vitrimer containing $0.13 \mathrm{~mol} \%$ bisdioxaborolane cross-links was investigated using different small strain rheological experiments (i.e., stress relaxation, SAOS, and creep). The addition of this small number of cross-links greatly affected the dynamics; it could not achieve steady-state flow within $8 \mathrm{~h}$. By isolating the soluble and insoluble portions, we determined that the vitrimer was essentially a composite of a viscoelastic fluid and solid. The insoluble phase behaved as a rubber that does not fully relax stress, while the soluble phase acted as a lubricant. Experiments on blended samples revealed that the macrophases not only have very different rheological properties, but they also interact in a synergistic manner to enhance the elasticity of the vitrimer. Hypotheses regarding the relationship between self-assembly and associative cross-linking are presented, and the repercussions on the processability of PE vitrimer (along with a small set of nonlinear observations) are discussed. We propose that when exposed to high shear stresses and strains, PE vitrimer can repeatedly fracture and heal, enabling it to flow.

\section{Experimental section}

Materials. High-density polyethylene (PE, melt flow index of $2.2 \mathrm{~g} / 10 \mathrm{~min}$ at $190{ }^{\circ} \mathrm{C}$ using a specified mass of $2.16 \mathrm{~kg}$ ), ethyl acetate, and 1,2-propanediol were obtained from Sigma-Aldrich; ethanol, hexane, tetrahydrofuran, and xylene were obtained from Carlo Erba Reagents; $( \pm) 3$ amino-1,2-propanediol, furan, and magnesium sulfate were obtained from Alfa Aesar; phenylboronic acid was obtained from TCI Chemicals; 1,4-diboronic acid was obtained from Activate Scientific. 
Synthesis of graft-functionalized polyethylene and polyethylene vitrimer. The syntheses of 1-[(2phenyl-1,3,2-dioxaborolan-4-yl)methyl]-1 $H$-pyrrole-2,5-dione (i.e., dioxaborolane maleimide) and 2,2'-(1,4-phenylene)-bis[4-methyl-1,3,2-dioxaborolane] (i.e., bis-dioxaborolane cross-linker) were conducted according to schemes reported in Röttger et al. ${ }^{21}$ For reactive mixing followed by extrusion of graft-functionalized PE (PE-g) and PE vitrimer (PE-v), the scheme reported in Ref. 56 was used. ${ }^{56}$ Table 1 details the measured graft/ethylene and cross-linker/graft molar ratios for PE- $g$ and PE- $v$. The functionalization and dynamic cross-linking of PE were achieved using a DSM Explore batch twin-screw extruder with a $5 \mathrm{~cm}^{3}$ capacity, co-rotating conical screw profile, and recirculation channel. To prepare PE- $g, 2.75 \mathrm{~g}$ of PE, $0.12 \mathrm{~g}$ of dioxaborolane maleimide, and $0.0014 \mathrm{~g}$ of dicumyl peroxide (DCP) were first mixed, then fed into the extruder, which was operating at a screw speed of $100 \mathrm{rpm}$ and preheated to a barrel temperature of $170{ }^{\circ} \mathrm{C}$. While in the extruder, the mixture was blanketed with nitrogen gas to minimize degradation. After a residence time of $8 \mathrm{~min}$, the functionalized polymer was discharged from the extruder. To prepare PE- $v$, the previously mentioned functionalization step was performed, but rather than releasing the polymer after the $8 \mathrm{~min}$ residence time, $0.12 \mathrm{~g}$ of bis-dioxaborolane maleimide was instead fed into the extruder. After an additional 6 min of residence time, the vitrimer was released from the extruder.

After extrusion, PE-g and PE- $v$ contain residual small molecules that can potentially evaporate under high temperatures (i.e., excess dioxaborolane maleimide, excess cross-linker, and boronic ester produced by the exchange reaction). Removal of these species is critical for improving the reproducibility of the rheological measurements. Accordingly, both samples were washed in dry acetone under reflux conditions at $60^{\circ} \mathrm{C}$. PE- $g$ and PE- $v$ were washed for 24 and $120 \mathrm{~h}$, respectively. To isolate the respective influences of the polymer and the addition of dicumyl peroxide, control samples of neat PE and PE $+0.05 \mathrm{wt} \% \mathrm{DCP}$ were also extruded and washed in acetone for $24 \mathrm{~h}$. 
Table 1. Graft/ethylene molar ratio, cross-linker/graft molar ratio, and insoluble fraction of polyethylene (PE), PE + $0.05 \mathrm{wt} \%$ dicumyl peroxide (DCP), graft-functionalized PE (PE-g), and $\mathrm{PE}$ vitrimer $(\mathrm{PE}-v)$.

\begin{tabular}{|c|c|c|c|}
\hline $\begin{array}{l}\text { Sample } \\
\text { name }\end{array}$ & $\begin{array}{r}\text { Measured } \\
{[\text { Graft }] /[\text { Ethylene }] \times} \\
1_{1000^{a, b}}\end{array}$ & $\begin{array}{r}\text { Measured } \\
{\text { [Cross-link }] /[\text { Graft }]^{a, b}}^{\text {Che }}\end{array}$ & $\begin{array}{r}\text { Insoluble } \\
\text { fraction } \\
\text { [total wt } \% \text { ] }\end{array}$ \\
\hline Polyethylene & 0 & - & 0 \\
\hline $\mathrm{PE}+0.05 \mathrm{wt} \% \mathrm{DCP}$ & 0 & - & 0 \\
\hline $\mathrm{PE}-g$ & $2.7 \pm 0.4$ & 0 & 0 \\
\hline $\mathrm{PE}-v$ & $2.6 \pm 0.2$ & $0.49 \pm 0.01$ & $33 \pm 1$ \\
\hline
\end{tabular}

${ }^{a}$ Determined by FTIR spectroscopy.

${ }^{b}$ Error bars are the standard deviation of measurements from three different pieces of the sample.

Isolation of insoluble and soluble portions of polyethylene vitrimer. As previously observed, ${ }^{15,21,56}$ PE vitrimer relinquishes an insoluble portion when placed in a good solvent. The insoluble portion of PE- $v$ (PE- $v$-insol) was isolated using a method based on the ISO 10147 standard procedure. 1.5 $\mathrm{g}$ of PE- $v$ was put inside a stainless steel fine wire cage and immersed in $345 \mathrm{~mL}$ of dried xylene. To minimize nondynamic covalent cross-linking of the PE backbone, $30 \mathrm{~g}$ of butylated hydroxytoluene antioxidant was added to the solution. The entire mixture was heated to reflux conditions at $140{ }^{\circ} \mathrm{C}$. After $18 \mathrm{~h}$, the sample cage was pulled out of the solution. To recover the soluble portion of PE- $v$ (PE- $v-S o l)$, the remaining extraction solution was concentrated under reduced pressure, reheated to $140{ }^{\circ} \mathrm{C}$, and then precipitated in dry acetone. Both PE- $v$-sol and PE$v$-insol were dried under reduced pressure at $140{ }^{\circ} \mathrm{C}$ for at least $12 \mathrm{~h}$. The dry mass of the PE-vinsol was measured to determine the insoluble fraction of PE- $v$. To certify that the vitrimer network was held together by dynamic covalent cross-links (rather than nondynamic linkages), $0.2 \mathrm{~g}$ of PE$v$-insol was subjected to a second extraction step. While the procedure was similar to the initial extraction step, $0.5 \mathrm{~mL}$ of 1,2-octanediol was added to the solution. Addition of the diol (which cleaves the bis-dioxaborolane cross-link) induced the entire sample to dissolve, thereby confirming 
the absence of nondynamic cross-links in the PE- $v$ network. The graft/ethylene and crosslinker/graft molar ratios of PE- $v$-sol and PE- $v$-insol are detailed in Table 2.

Table 2. Graft/ethylene and cross-linker/graft molar ratios of PE- $v$-sol and PE- $v$-insol.

\begin{tabular}{lrr}
\hline $\begin{array}{l}\text { Sample } \\
\text { name }\end{array}$ & $\begin{array}{r}\text { Measured } \\
\text { [Graft]/[Ethylene] } \times \\
\mathbf{1 0 0 0}^{\boldsymbol{a}, \boldsymbol{b}}\end{array}$ & $\begin{array}{r}\text { Measured } \\
\text { [Cross-link]/[Graft] }]^{\boldsymbol{a}, \boldsymbol{b}}\end{array}$ \\
\hline PE- $v$-sol & $1.1 \pm 0.1$ & 0 \\
PE- $v$-insol & $5.1 \pm 0.4$ & $0.51 \pm 0.02$ \\
\hline${ }^{a}$ Determined by FTIR spectroscopy. & & \\
${ }^{\boldsymbol{b}}$ Error bars are the standard deviation of measurements from three different pieces of the sample.
\end{tabular}

Preparation of blend PE/PE-v-insol and blend PE-v-sol/PE-v-insol. Blends PE/PE-v-insol and PE$v$-sol/PE-v-insol were prepared using the previously described DSM Explore batch twin-screw extruder. The former blend was prepared using a feed mixture of $1.84 \mathrm{~g}$ of PE and $0.91 \mathrm{~g} \mathrm{PE}-v-$ insol, while the latter used a feed of $1.84 \mathrm{~g}$ of PE-v-sol and $0.91 \mathrm{~g}$ of PE-v-insol. For each blend, the feed mixture was charged into the extruder, which was pre-heated to $170{ }^{\circ} \mathrm{C}$ and operating at a screw speed of $100 \mathrm{rpm}$. After a residence time of $8 \mathrm{~min}$, during which the extruder barrel was inundated with nitrogen gas to minimize sample degradation, the blend was released from the extruder. The graft/ethylene molar ratio, cross-linker/graft molar ratio, and fraction of PE- $v$-insol (as determined from material balances) for both blends are listed in Table 3.

Table 3. Graft/ethylene molar ratio, cross-linker/graft molar ratio, and PE-v-insol fraction of PE vitrimer blends.

\begin{tabular}{|c|c|c|c|}
\hline $\begin{array}{l}\text { Sample } \\
\text { name }\end{array}$ & $\begin{array}{r}\text { Measured } \\
\text { [Graft]/[Ethylene] } \\
\times 1000^{a, b}\end{array}$ & $\begin{array}{r}\text { Measured } \\
\text { [Cross-link]/[Graft] }^{a, b}\end{array}$ & $\begin{array}{r}\text { PE-v-insol } \\
\text { fraction } \\
{\left.\text { [total } w \%^{c}\right]^{c}}^{\text {total }}\end{array}$ \\
\hline Blend PE/PE-v-insol & $1.9 \pm 0.2$ & $0.51 \pm 0.01$ & $38 \pm 5$ \\
\hline Blend PE- $v$-sol/PE- $v$-insol & $2.2 \pm 0.1$ & $0.50 \pm 0.02$ & $29 \pm 5$ \\
\hline
\end{tabular}

${ }^{a}$ Determined by FTIR spectroscopy.

${ }^{b}$ Error bars are the standard deviation of measurements from three different pieces of the sample. ${ }^{c}$ Error bars were calculated from propagated error analysis. 
Quantification of composition. Graft/ethylene and cross-linker/graft molar ratios for all samples were determined by using Fourier transform infrared spectroscopy (FTIR). A Bruker Tensor 37 spectrometer rigged with a Specac Goldengate attenuated total reflection cell was used for measurements. $\mathrm{CH}_{2}$ and $\mathrm{CH}_{3}$ scissoring signals of the PE backbone appeared at 1470 and 1450 $\mathrm{cm}^{-1}$, respectively. The $\mathrm{C}=\mathrm{O}$ stretching peak of dioxaborolane maleimide occurred at $1710 \mathrm{~cm}^{-1}$, while the aromatic $\mathrm{C}=\mathrm{C}$ stretching peaks appeared at 1600 and $1500 \mathrm{~cm}^{-1}$. For the bisdioxaborolane cross-linker, the aromatic $\mathrm{C}=\mathrm{C}$ stretching signal showed at $1517 \mathrm{~cm}^{-1}$. FTIR spectra were measured in absorbance mode and decomposed by fitting the characteristic peaks to skewed Gaussian functions. The average graft/ethylene and cross-linker/graft molar ratios were quantified using eqns. 1-3:

$$
\begin{gathered}
\frac{C_{\text {graft }}}{C_{P E}}=\frac{A_{1710}}{A_{1470}} \frac{\varepsilon_{1470}}{\varepsilon_{1710}} \frac{\lambda_{1470}}{\lambda_{1710}} \\
\frac{C_{\text {cross-link }}}{C_{\text {graft }}}=\frac{A_{1517}}{A_{1710}} \frac{\varepsilon_{1710}}{\varepsilon_{1517}} \frac{\lambda_{1710}}{\lambda_{1517}} \\
\frac{C_{\text {cross-link }}}{C_{P E}}=\frac{C_{\text {crosslink }}}{C_{\text {graft }}} \times \frac{C_{\text {graft }}}{C_{P E}}
\end{gathered}
$$

where $C_{i}$ is the molar concentration of component $i, \lambda_{j}$ is wavenumber $j, A_{j}$ is the area under the peak at wavenumber $j$, and $\varepsilon_{j}$ is the absorption coefficient of the peak at wavenumber $j . \varepsilon_{1710}$, $\varepsilon_{1517}$, and $\varepsilon_{1470}$ were determined previously. ${ }^{56}$

Rheology measurements. Linear viscoelastic properties of all samples were evaluated by using an Anton Paar MCR501 rotational rheometer equipped with a $25 \mathrm{~mm}$ parallel plate geometry. The upper and lower geometries are a stainless steel plate and a disposal aluminum plate, respectively. To minimize thermal degradation, the plate geometry was enclosed in a convection oven under a $200 \mathrm{~L} / \mathrm{h}$ nitrogen flow. The oven achieved a temperature control accuracy of $\pm 0.5^{\circ} \mathrm{C}$. During all 
measurements, a normal force of $0.1 \mathrm{~N}$ was imposed to maintain contact between the upper plate and the sample. Rheology samples were prepared by compression molding. Samples were molded into disc shapes (25 mm diameter and $1.5 \mathrm{~mm}$ thickness) using stainless steel frames, which were subjected to an applied load of 3 metric tons at $150{ }^{\circ} \mathrm{C}$ for $8 \mathrm{~min}$.

Strain sweeps, small-amplitude oscillatory shear (SAOS), stress relaxation, and creep compliance and recovery measurements were performed. Strain sweeps were used to determine the linear viscoelastic regimes that were to be probed during SAOS and stress relaxation experiments (see Figures S2 and S3). For each sample, the critical strain value was identified at $140{ }^{\circ} \mathrm{C}$. Subsequent SAOS and stress relaxation measurements on fresh samples at all temperatures were performed using an imposed strain that was an order of magnitude lower than the critical strain observed at $140{ }^{\circ} \mathrm{C}$. For example, the critical strain at $140{ }^{\circ} \mathrm{C}$ was measured to be $120 \%$ for PE and 100\% for PE- $v$. Therefore, SAOS and stress relaxation measurements between 180-140 ${ }^{\circ} \mathrm{C}$ were performed using a strain of $5 \%$ for PE and $1 \%$ for PE- $v$. SAOS measurements were run at an angular frequency $(\omega)$ range from 100 to $0.01 \mathrm{rad} / \mathrm{s}$. Time-temperature superposition was performed on the PE SAOS data using a reference temperature of $160{ }^{\circ} \mathrm{C}$. The horizontal shift factors $\left(a_{T}\right)$ were determined visually by horizontally moving the $\tan \delta$ data, and then were fit to an Arrhenius-type equation

$$
\ln \left(a_{T}\right)=\frac{\Delta E_{a c t}}{R}\left(\frac{1}{T}-\frac{1}{T_{r e f}}\right)
$$

where $\Delta E_{\text {act }}$ is the flow activation energy, $R$ is the gas constant, $T$ is the measurement temperature, and $T_{r e f}$ is the reference temperature of $160^{\circ} \mathrm{C} .{ }^{96}$ van Gurp-Palmen plots were also prepared to determine if samples obeyed time-temperature superposition (see Supporting Information). ${ }^{97}$

For creep and recovery measurements, a shear stress was first applied for up to $8 \mathrm{~h}$. Subsequently, the stress was removed, and the sample was allowed to recover for $2 \mathrm{~h}$. The creep compliance $(J(t))$ and creep recovery compliance $\left(J_{r}(t)\right)$ were determined using the formula 


$$
\begin{gathered}
J(t)=\gamma(t) / \sigma \\
J_{r}(t)=\left(\gamma_{f}-\gamma(t)\right) / \sigma
\end{gathered}
$$

where $\gamma(t)$ is strain, $\sigma$ is the stress applied during creep, and $\gamma_{f}$ is the value of the strain when $\sigma$ was removed. To eliminate the parasitic drift from the creep recovery compliance, the true creep recovery compliance $\left(J_{r}^{\text {true }}(t)\right)$ was estimated by

$$
J_{\mathrm{r}}^{\text {true }}(t)=J_{r}(t)-\frac{d J_{r}}{d t_{r}} t_{r}
$$

where $t_{r}$ is the time since recovery started and $\frac{d J_{r}}{d t_{r}}$ is the linear slope of $J_{r}(t) \cdot{ }^{95}$ For samples that achieved steady-state flow $\left(J(t) \sim t^{1}\right)$, several material properties were estimated. The steadystate linear recoverable compliance $\left(J_{e}^{0}\right)$, which describes the elasticity of the sample, was estimated from the plateau of $J_{\mathrm{r}}^{t r u e}(t)$. The zero-shear viscosity $\left(\eta_{0}\right)$, was determined by fitting the linear portion of $J(t)$ to

$$
J(t)=\frac{t}{\eta_{0}}+J_{e}^{0}
$$

Because $J_{e}^{0}$ was determined from the $J_{\mathrm{r}}^{\text {true }}(t)$ data, the only fitting parameter in Eqn. 8 was $\eta_{0}$. The terminal relaxation time $(\tau)$ was then estimated by

$$
\tau=J_{e}^{0} \eta_{0}
$$

Similar to $a_{T}, \tau$ can be fit to an Arrhenius-type equation 


$$
\ln (\tau)=\frac{\Delta E_{a c t}}{R}\left(\frac{1}{T}-\frac{1}{T_{r e f}}\right)+\ln \left(\tau_{r e f}\right)
$$

where $\Delta E_{\text {act }}$ is the flow activation energy, $R$ is the gas constant, $T$ is the measurement temperature, $T_{r e f}$ is the reference temperature of $160{ }^{\circ} \mathrm{C}$, and $\tau_{r e f}$ is the relaxation time at $160{ }^{\circ} \mathrm{C} .{ }^{95} J_{\mathrm{r}}^{\text {true }}(t)$, $J_{e}^{0}, \eta_{0}$, and $\tau$ for PE, PE $+0.05 \mathrm{wt} \% \mathrm{DCP}, \mathrm{PE}-g$, and PE- $v$-sol are provided in the Supporting Information.

For most samples, the typical experiment protocol was as follows. The sample disc was placed onto the lower plate, which was pre-heated to $185^{\circ} \mathrm{C}$. The upper plate and convection oven were then slowly lowered onto the sample to form a plate gap of approximately $1.5 \mathrm{~mm}$. The sample was then cooled at a rate of $1^{\circ} \mathrm{C} / \mathrm{min}$ to $180^{\circ} \mathrm{C}$, annealed for $30 \mathrm{~min}$, and then SAOS was performed. At the conclusion of SAOS, the sample was annealed for another $30 \mathrm{~min}$, and then a stress relaxation measurement was conducted. Subsequent measurements at 170, 160, 150, and $140{ }^{\circ} \mathrm{C}$ followed the same scheme. A similar procedure was used for creep compliance and recovery measurements, except measurements were only performed at 180,160 , and $140{ }^{\circ} \mathrm{C}$. Our previous study demonstrated that at $160{ }^{\circ} \mathrm{C}$ a PE vitrimer sample with $1.2 \mathrm{~mol} \%$ of dioxaborolane maleimide grafts produced identical small-angle X-ray scattering patterns after annealing periods of $5 \mathrm{~min}$ and $48 \mathrm{~h} .{ }^{56}$ Therefore, during the $30 \mathrm{~min}$ annealing periods (applied before each measurement), the structure of PE vitrimer likely should not change significantly. Some thermal degradation during long rheological experiments was observed in the cases of PE-g and blend PE$v$-sol/PE-v-insol at the highest studied temperatures $\left(180^{\circ} \mathrm{C}\right)$. Therefore, for these samples an alternative experiment protocol was conducted. Measurements were first performed at $140{ }^{\circ} \mathrm{C}$, and then at subsequently higher temperatures. Comparisons between the data obtained by the two protocols are in the Supporting Information. For PE-v, PE- $v$-insol, blend PE/PE-v-insol, and blend PE-v-sol/PE-v-insol, portions of the samples used for rheology were subjected to extraction in xylene in the presence of excess 1,2-octanediol. All samples completely dissolved, suggesting that 
they did not form a nondynamic covalently cross-linked network during the rheology measurements.

\section{Results}

Quantification of graft/ethylene and cross-linker/graft molar ratios. Graft/ethylene and crosslinker/graft molar ratios of the samples, determined using FTIR spectroscopy, are listed in Tables 1,2 , and 3. The neat PE, PE $+0.05 \mathrm{wt} \%$ DCP, and PE-g FTIR spectra - displayed in Figure S1A

- feature a $\mathrm{CH}_{2}$ scissoring peak (characteristic of the polymer backbone) and a $\mathrm{CH}_{3}$ scissoring peak (representing branches in the chain). The PE-g spectrum also exhibits a $\mathrm{C}=\mathrm{O}$ stretch signal at $1710 \mathrm{~cm}^{-1}$ that reflects the maleimide portion of the graft, and two aromatic $\mathrm{C}=\mathrm{C}$ stretching peaks at 1600 and $1500 \mathrm{~cm}^{-1}$ that stem from the phenyl ring. The PE- $v$ spectrum (Figure 3 ) exhibits the polyolefin and maleimide signals, but does not display the graft aromatic peaks. Instead, it only has a single peak at $1520 \mathrm{~cm}^{-1}$ that is representative of aromatic $\mathrm{C}=\mathrm{C}$ stretching in the cross-linker. The 1:2 cross-linker/graft molar ratio suggests the dioxaborolane maleimide grafts in the vitrimer are saturated with cross-linker.

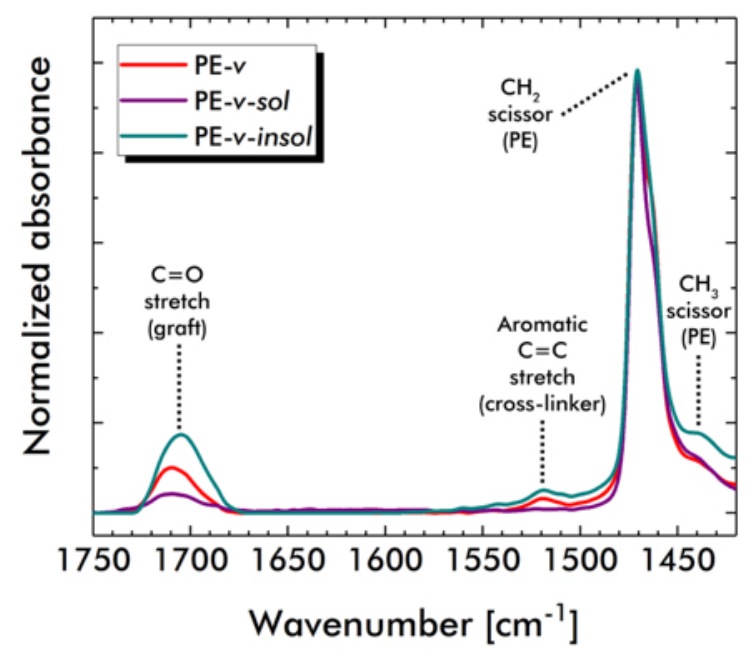

Figure 3. FTIR spectra of PE-v, PE- $v$-sol, and $\mathrm{PE}-v$-insol. Characteristic signals of the $\mathrm{PE}$ backbone, graft, and cross-linker components are highlighted. 
The FTIR spectra of PE- $v$-insol and PE- $v-$ sol show that these portions of the vitrimer have very different compositions (Figure 3). $\mathrm{PE}-\mathrm{v}$-insol has stronger $\mathrm{C}=\mathrm{O}$ and aromatic $\mathrm{C}=\mathrm{C}$ stretching signals than PE- $v$, suggesting that it is richer in both graft and cross-linker. The graft/ethylene molar ratio is larger by a factor of approximately 2 , while the 1:2 cross-linker/graft molar ratio was retained. The cross-link density estimated from FTIR $\left(v_{F T I R}\right)$ for PE- $v$-insol is $4.7 \times 10^{-2} \mathrm{~nm}^{-3}$. Conversely, $\mathrm{PE}-\boldsymbol{v}$-sol has a weaker $\mathrm{C}=\mathrm{O}$ stretch signal and lower graft/ethylene molar ratio than the initial vitrimer. The aromatic $\mathrm{C}=\mathrm{C}$ stretch signal is not observed. The absence of the peak, however, does not conclusively prove PE-v-sol lacks cross-linker; the signal could possibly be below the detection limit of FTIR. The measured graft/ethylene molar ratios of the PE-v-insol and PE- $v$-sol agree with the material balance on the initial vitrimer.

The FTIR spectra for blends PE/PE- - -insol and PE- $v$-sol/PE- $v$-insol are seen in Figure S1B, and their compositions are detailed in Table 3. The weight fractions of PE-v-insol in the blends, as determined by material balances, are comparable to the insoluble fraction of PE-v.

Polyethylene, PE-g, and PE-v rheology. The rheology of the neat PE used in this study is presented in Figure 4. It behaves as a viscoelastic fluid. The molecular weight and dispersity of this commercial polymer $\left(M_{n}=11 \mathrm{~kg} / \mathrm{mol}\right.$ and $\left.\mathrm{Ð}=7.5\right)$ were reported previously. ${ }^{56}$ Figure $4 \mathrm{~A}$ features the horizontal shift factors $\left(a_{T}\right)$ determined by shifting the PE SAOS $\tan \delta$ data (Figure S4C). By fitting $a_{T}$ values to Eqn. 4, a flow activation energy $\left(\Delta E_{a c t}\right)$ of $15.5 \pm 0.2 \mathrm{~kJ} / \mathrm{mol}$ was estimated. $a_{T}$ values were then applied to the SAOS, stress relaxation, and creep compliance data to form the master curves seen in Figures 4B, 4C, and 4D, respectively. (Unshifted data are shown in Figures S4A, S4B, S5, and S7). The collapse of the data in each curve (and in the van GurpPalmen plot in Figure S4D) confirms that the PE obeys time-temperature superposition. The elastic and viscous moduli in Figure 4B did not achieve the expected terminal frequency scalings of -2 and -1, likely due to the large molecular weight dispersity of the PE. Therefore, the zero-shear viscosities $\left(\eta_{0}\right)$ and terminal relaxation times of PE $(\tau)$ were estimated from creep compliance. At 
all temperatures, PE exhibits the steady-state flow time scaling of 1, showing that the material achieved terminal relaxation. The steady-state linear recoverable compliance $\left(J_{e}^{0}\right)$, which describes the elasticity of the sample, is approximately $2 \times 10^{-3} \mathrm{~Pa}^{-1}$ at all temperatures (Figure S9A). Based on eqns. 8 and $9, \eta_{0}$ spans from $1.2 \times 10^{4}$ to $1.6 \times 10^{4} \mathrm{~Pa} \mathrm{~s}$, and $\tau$ ranges from 23 to $36 \mathrm{~s}$ (Figures S9B and S9C). By fitting $\tau$ to Eqn. 10 , an $\Delta E_{\text {act }}$ of $16 \pm 6 \mathrm{~kJ} / \mathrm{mol}$ was estimated (Figure S9D), consistent with the value determined from $a_{T}$.
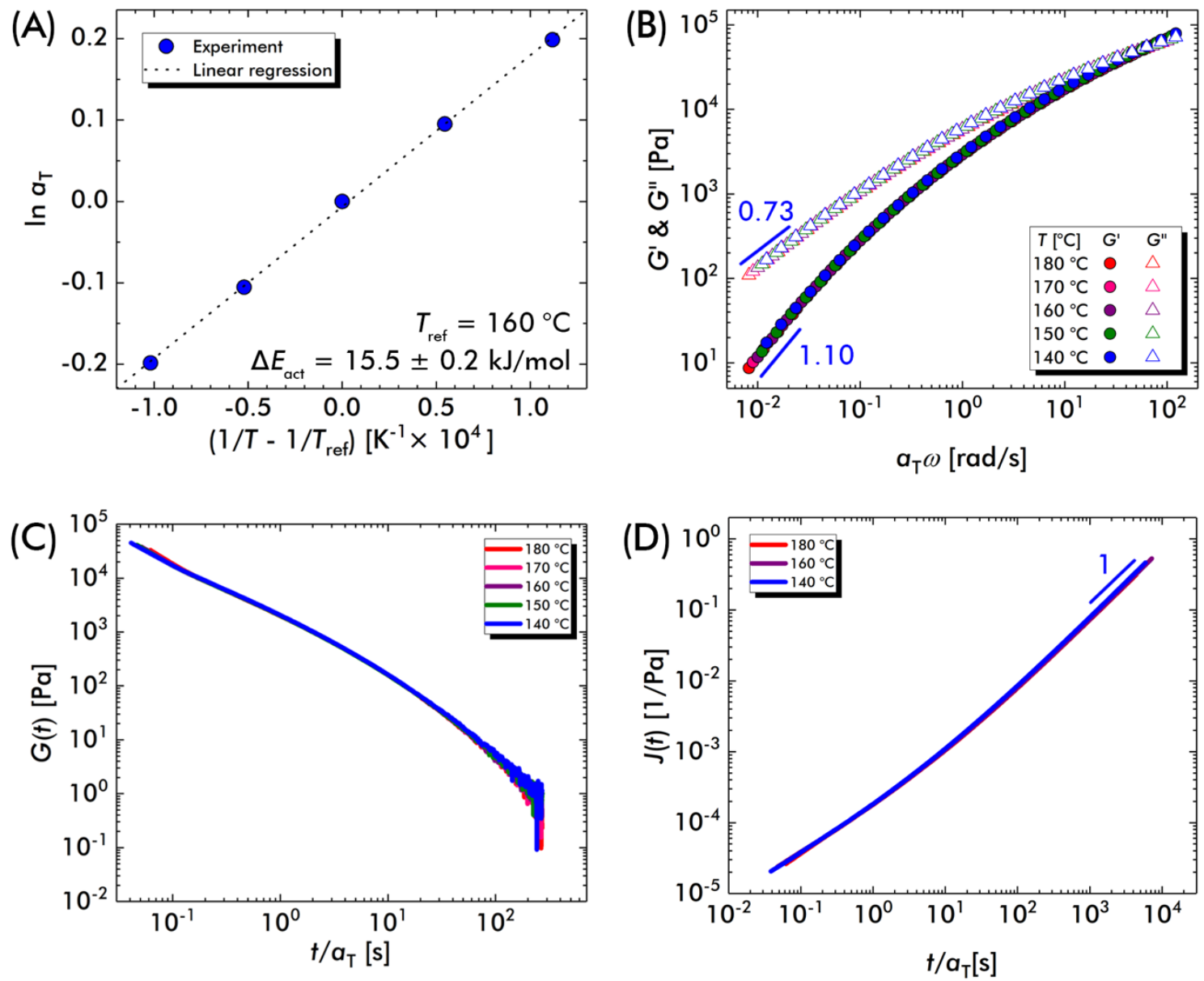

Figure 4. (A) Time-temperature superposition shift factors for neat PE. Linear regression estimates an activation energy of $15.5 \pm 0.2 \mathrm{~kJ} / \mathrm{mol}$. (B) Small-amplitude oscillatory shear, (C) stress relaxation, and (D) creep compliance master curves. For all master curves, $T_{\text {ref }}=160{ }^{\circ} \mathrm{C}$.

$\mathrm{PE}+0.05 \mathrm{wt} \% \mathrm{DCP}-$ whose rheology is detailed in the Supporting Information - is still a viscoelastic liquid, but it relaxes significantly more slowly than neat PE. Its $\tau$ spans from 440 to 
680 s. This sample also does not obey time-temperature superposition. The differences between its flow properties and those of neat PE are attributed to the formation of branched chains. However, the $\Delta E_{\text {act }}$ determined from creep is $16 \pm 1 \mathrm{~kJ} / \mathrm{mol}$, matching the value of the virgin polymer (Figure S14D). These findings are consistent with the observations of van Gurp and Palmen. ${ }^{97}$

The rheology of PE- $g$ is presented in Figure 5. Although it only contains the dioxaborolane maleimide grafts and no cross-linker, it still macro- and microphase separates. ${ }^{56}$ As such, the PE$g$ rheological behavior drastically deviates from neat $\mathrm{PE}$ and $\mathrm{PE}+0.05 \mathrm{wt} \% \mathrm{DCP}$. The SAOS data for PE- $g$ do not obey time-temperature superposition (Figure S15). The elastic and viscous moduli are nearly equal at high $\omega$, but $G^{\prime}$ begins to decrease faster than $G^{\prime \prime}$ at $\omega<1 \mathrm{rad} / \mathrm{s}$ (Figure 5A). During stress relaxation (Figure 5B), the modulus decreases by about three orders of magnitude within $1000 \mathrm{~s}$, then follows power law behavior at longer times. Creep measurements show that the PE- $g$ flow behavior is sensitive to temperature. At $140{ }^{\circ} \mathrm{C}, J(t)$ only reaches a power law scaling of 0.76 within $8 \mathrm{~h}$ (Figure 5C). Even when the measurement is extended to $20 \mathrm{~h}$, the sample still does not achieve steady-state flow (Figure S17). When the temperature is elevated to 160 and $180^{\circ} \mathrm{C}$, however, it exhibits steady-state flow (Figure 5C). $J_{e}^{0}$ ranges from $3.4 \times 10^{-3}$ to $4.2 \times 10^{-3}$ $\mathrm{Pa}^{-1}$ (Figure S19A), which are in between the PE and PE $+0.05 \mathrm{wt} \%$ DCP values. $\eta_{0}$ and $\tau$ are significantly larger for PE-g than for the control samples (Figure S19B and S19C). In particular, $\tau$ spans from 2000 to $2900 \mathrm{~s}$. 

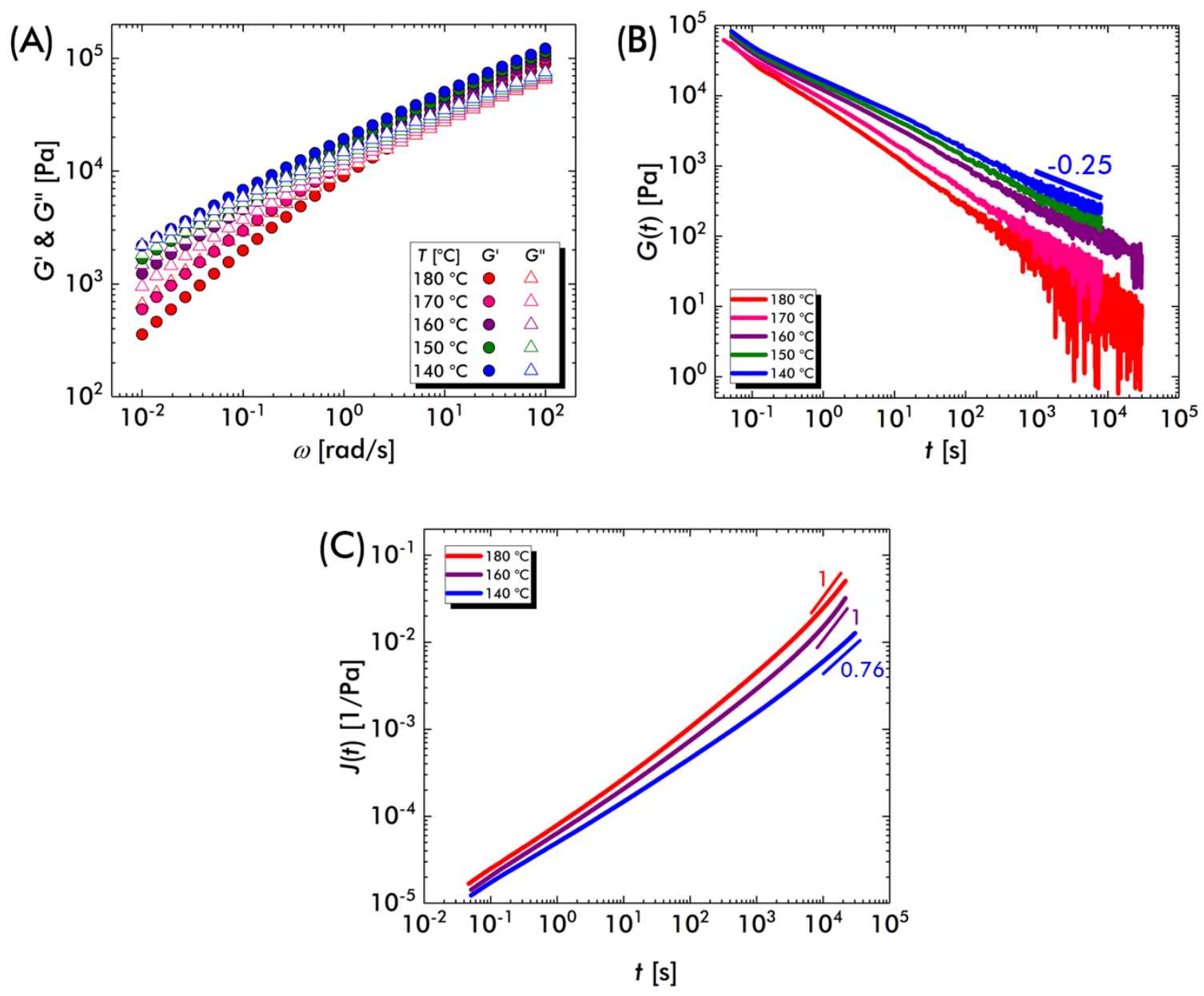

Figure 5. (A) SAOS, (B) stress relaxation, and (C) creep compliance measurements of PE- $g$.

The rheology of PE- $v$ is presented in Figure 6. Both self-assembly and associative crosslinking dictate the linear viscoelastic behavior of the vitrimer. During SAOS (Figure 6A), the elastic modulus $\left(G^{\prime}\right)$ is larger than the viscous modulus $\left(G^{\prime \prime}\right)$ over the entire $\omega$ range, and approaches a plateau value of $\sim 10^{4} \mathrm{~Pa}$ at $\omega=10^{-2} \mathrm{rad} / \mathrm{s}$. Both $G^{\prime}$ and $G^{\prime \prime}$ are marginally sensitive to temperature and do not obey time-temperature superposition. Stress relaxation measurements shows that at longer times $(\mathrm{t}>1000 \mathrm{~s}) \mathrm{PE}-\mathrm{v}$ begins to further relax (Figure 6B and S20). The decay rate decreases as the temperature is decreased. Creep measurements demonstrate that PE-v does not achieve terminal relaxation within $8 \mathrm{~h}$. As seen in Figure $6 \mathrm{C}$, the PE- $v$ creep compliance data 
only reach a power law scaling of $\leq 0.37$, much lower the linear time scaling of steady-state flow. Because the vitrimer does not achieve terminal relaxation, $J_{e}^{0}, \eta_{0}$, and $\tau$ could not be estimated.
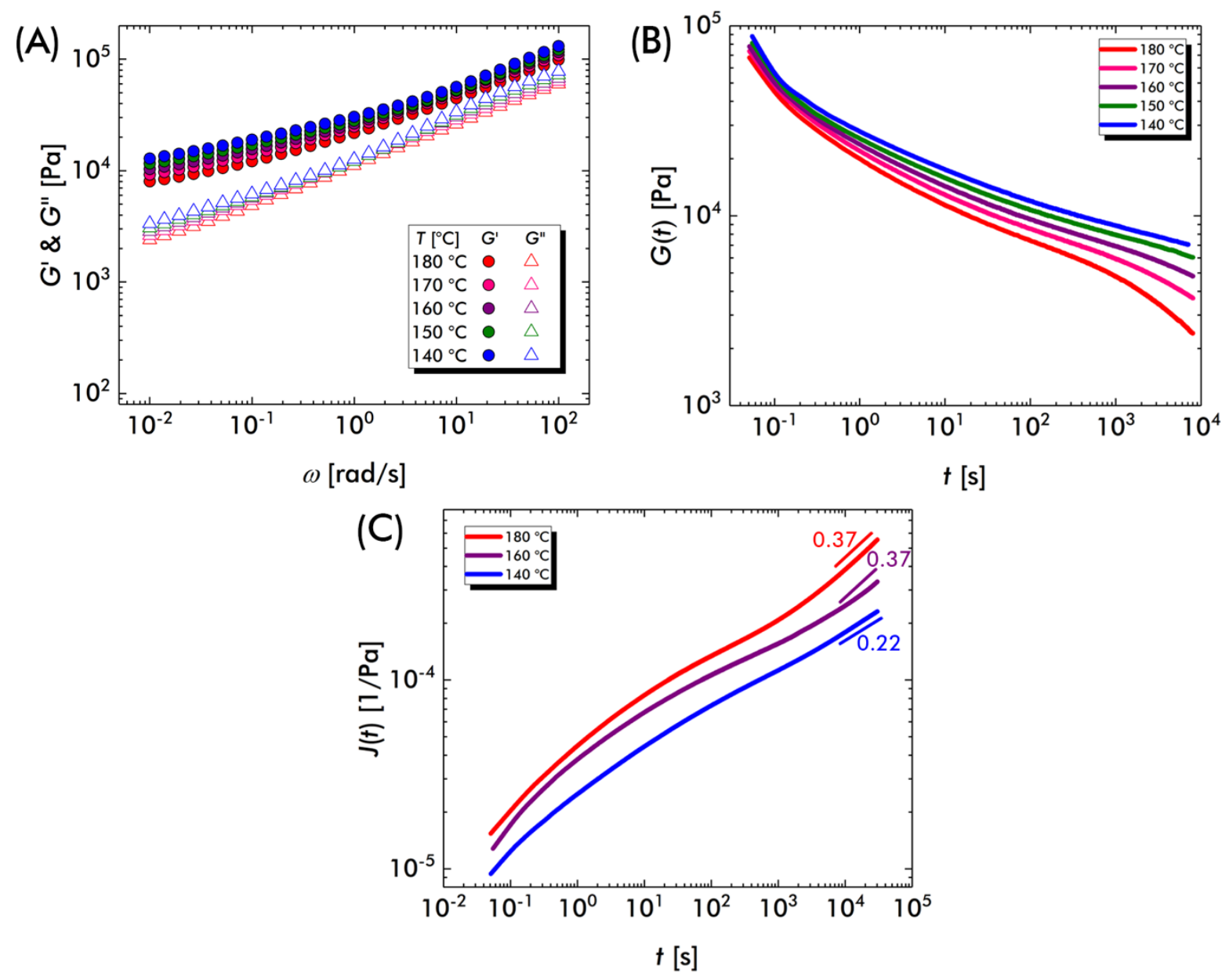

Figure 6. (A) SAOS, (B) stress relaxation, and (C) creep compliance measurements of PE- $v$.

These observations not only show that aggregation of dioxaborolane maleimide slows down the relaxation of the PE chains, but also reveal that the addition of associative cross-links hinders the vitrimer from achieving steady-state flow.

$P E$-v-sol and PE-v-insol rheology. PE vitrimer is effectively an immiscible blend of graft-poor and graft-rich domains. ${ }^{56}$ To understand how these distinct regions impact linear viscoelasticity, the rheological properties of PE- $v$-sol and PE- $v$-insol were separately explored. 
The rheology of PE-v-sol is presented in Figure 7. It behaves as a viscoelastic fluid. During SAOS, $G^{\prime \prime}$ was larger than $G^{\prime}$ at all $\omega$ values (Figure S23A). At low $\omega$, however, $G^{\prime}$ increases as the temperature decreases, leading to lower values of $\tan \delta$ (Figure S23B). This sensitivity to temperature is also seen during stress relaxation. At $180{ }^{\circ} \mathrm{C}$, the PE- $v$-sol $G(t)$ was very similar to that of neat PE (Figures S24 and S39). As the temperature is decreased, a plateau in the relaxation modulus appears at $t>3 \mathrm{~s}$. This plateau becomes more apparent as the temperature is decreased. During creep, $J(t)$ achieves the steady-state flow scaling of 1 at all temperatures (Figure 7A). $J_{e}^{0}$ averages around $9.5 \times 10^{-3} \mathrm{~Pa}^{-1}$ (similar to the PE $+0.05 \mathrm{wt} \%$ DCP values), while $\eta_{0}$ decreases from $3.4 \times 10^{4}$ to $1.4 \times 10^{4} \mathrm{~Pa} \mathrm{~s}$ as the temperature increased (Figures S27A and S27B). $\tau$ diminishes from 310 to $140 \mathrm{~s}$ and expresses an $\Delta E_{\text {act }}$ of $32 \pm 2 \mathrm{~kJ} / \mathrm{mol}$, which is twice as large as the values for PE and PE $+0.05 \mathrm{wt} \%$ DCP (Figures 7B and S27C).
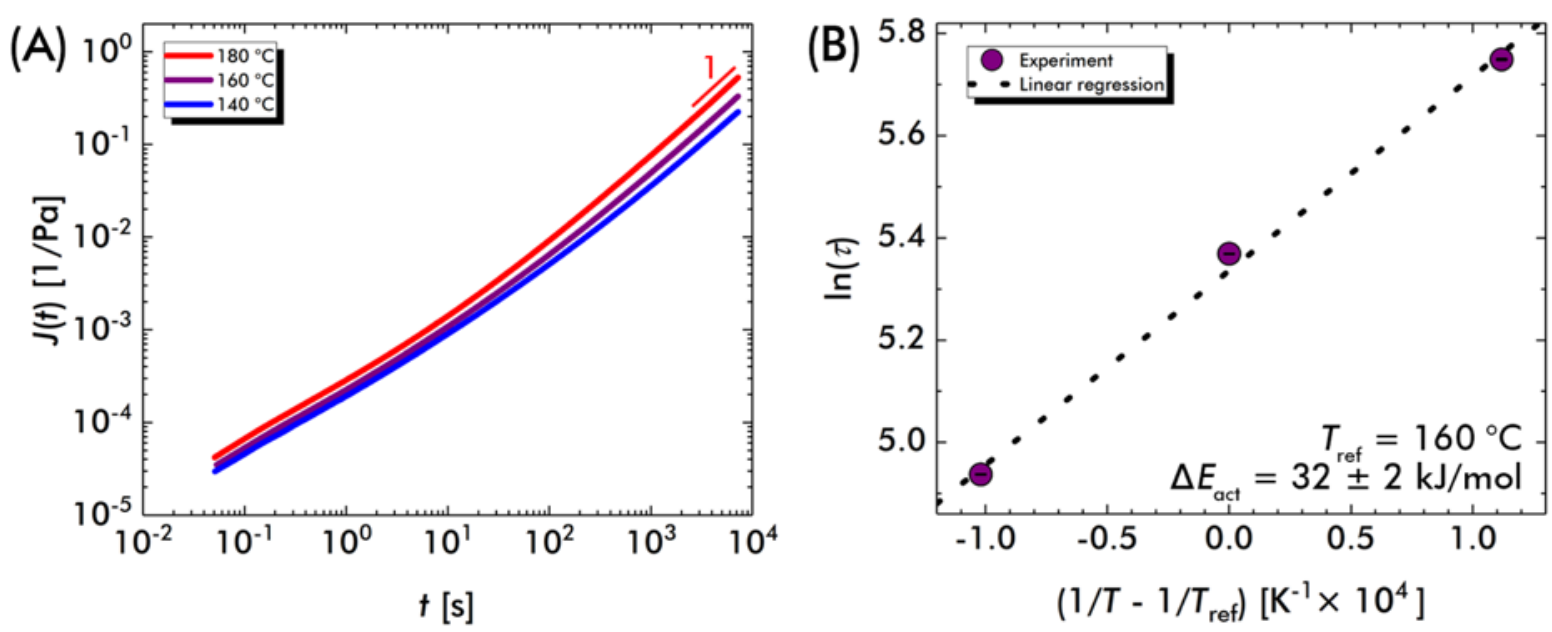

Figure 7. (A) Creep compliance measurements of PE- $v$-sol. At all temperatures, $\sigma=10 \mathrm{~Pa}$. (B) Linear regression of the PE- $v$-sol relaxation times to Eqn. 10. An activation energy of $32 \pm 2$ $\mathrm{kJ} / \mathrm{mol}$ is estimated.

The rheology of PE- $v$-insol is presented in Figure 8. In contrast to the soluble material, PE$v$-insol is essentially a viscoelastic solid. As seen in the SAOS data in Figure 8A, both the elastic and viscous moduli plateau over the entire $\omega$ range. Averaged over all $\omega$ and temperatures, this plateau corresponds to a mean complex modulus $\left(G^{*}\right)$ of $2.8 \times 10^{4} \mathrm{~Pa}$, which is lower than the 
entanglement modulus of PE $\left(2 \times 10^{6} \mathrm{~Pa}\right) .{ }^{98}$ The complex modulus value is consistent with the low cross-link density measured by FTIR. During stress relaxation, the material exhibits power law behavior at $t<100 \mathrm{~s}$ for all temperatures (Figure S29). At $180{ }^{\circ} \mathrm{C}, G(t)$ begins to relax more quickly at $t \sim 10^{4} \mathrm{~s}$. This relaxation process, however, is much slower at lower temperatures. The high elasticity of PE- $v$-insol is further observed during creep. $J(t)$ curves feature damped oscillations at $t<1 \mathrm{~s}$ (Figure 8B), resulting from the coupling of sample elasticity with the rheometer inertia at the start of the measurement. ${ }^{99}$ Throughout the $8 \mathrm{~h}$ creep, PE- $v$-insol relaxes very slowly and remains far from achieving steady-state flow. $J(t)$ reaches a scaling of 0.20 at 180 ${ }^{\circ} \mathrm{C}$, while it remains virtually flat at $140{ }^{\circ} \mathrm{C}$. The upturn in the $J(t)$ data at $180{ }^{\circ} \mathrm{C}$ is consistent with the downturn seen in the $G(t)$ at the same temperature.
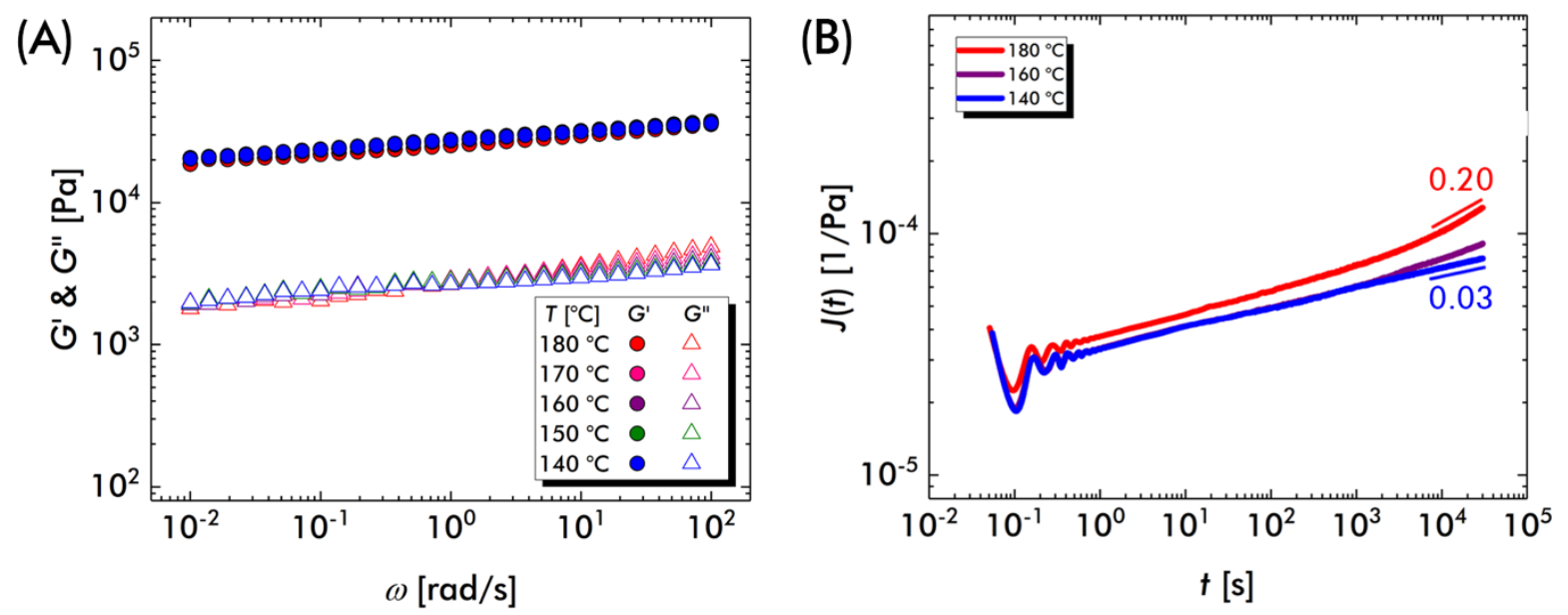

Figure 8. (A) SAOS and (B) creep compliance data of PE-v-insol. For (B), $\sigma=500$ Pa was used for all temperatures.

The complex rheology of PE- $v$ is significantly influenced by its distinct macrophases. The two components that comprise PE- $v$ not only differ in their compositions, but also express strikingly dissimilar flow properties. 


\section{Discussion}

1) Utility of creep and SAOS measurements on vitrimer systems. Vitrimers are interesting engineering materials that combine the useful properties of both thermosets and thermoplastics; they are solvent resistant, yet still processable. A deep understanding of vitrimer rheology is critical for enabling their use in high-end applications. The viscoelasticity of vitrimer materials has typically been evaluated using only stress relaxation measurements, in which the terminal relaxation time $(\tau)$ is estimated by assuming that the relaxation modulus $(G(t))$ follows an exponential form. ${ }^{54}$ Although this protocol is convenient, it may lead to misjudgement of the processability of a vitrimer. Stress relaxation data inherently become noisier as the measurement proceeds towards the torque limit of the rheometer. If a low step strain is needed to stay in the linear viscoelastic regime, the resulting $G(t)$ may be too noisy to precisely observe rheological features. Moreover, interpretation of stress relaxation data is significantly influenced by how the data are plotted. For example, one common practice in the vitrimer literature is to plot a normalized $G(t)$ on linear or semi-logarithmic axes. $\tau$ is taken to be the time in which the normalized $G(t)$ reaches $1 / e .^{54}$ This sort of analysis, however, has no theoretical justification. The $G(t)$ measured by stress relaxation is a convolution of several relaxation modes occurring over a wide range of time scales and with different temperature dependencies (e.g., Rouse, reptation, dynamic crosslinking). The absolute values of $G(t)$ also reveal information about the covalent and physical cross-link densities. ${ }^{55,75}$ To capture the wealth of information afforded by stress relaxation measurements, $G(t)$ should be plotted in a non-normalized manner on logarithmic axes. To emphasize this point, we normalized the stress relaxation data for PE from Figure $6 \mathrm{~B}$ and replotted them on semi-logarithmic axes (Figure 9). The data were normalized using the value of $G(t=$ $0.05 \mathrm{~s}$ ), which is comparable to the $G^{*}$ values measured during SAOS at high $\omega$. Based on the $1 / e$ criterion, Figure 9B suggests that PE- $v$ fully relaxes in less than 0.5 seconds, greatly exaggerating the processability of the material. 

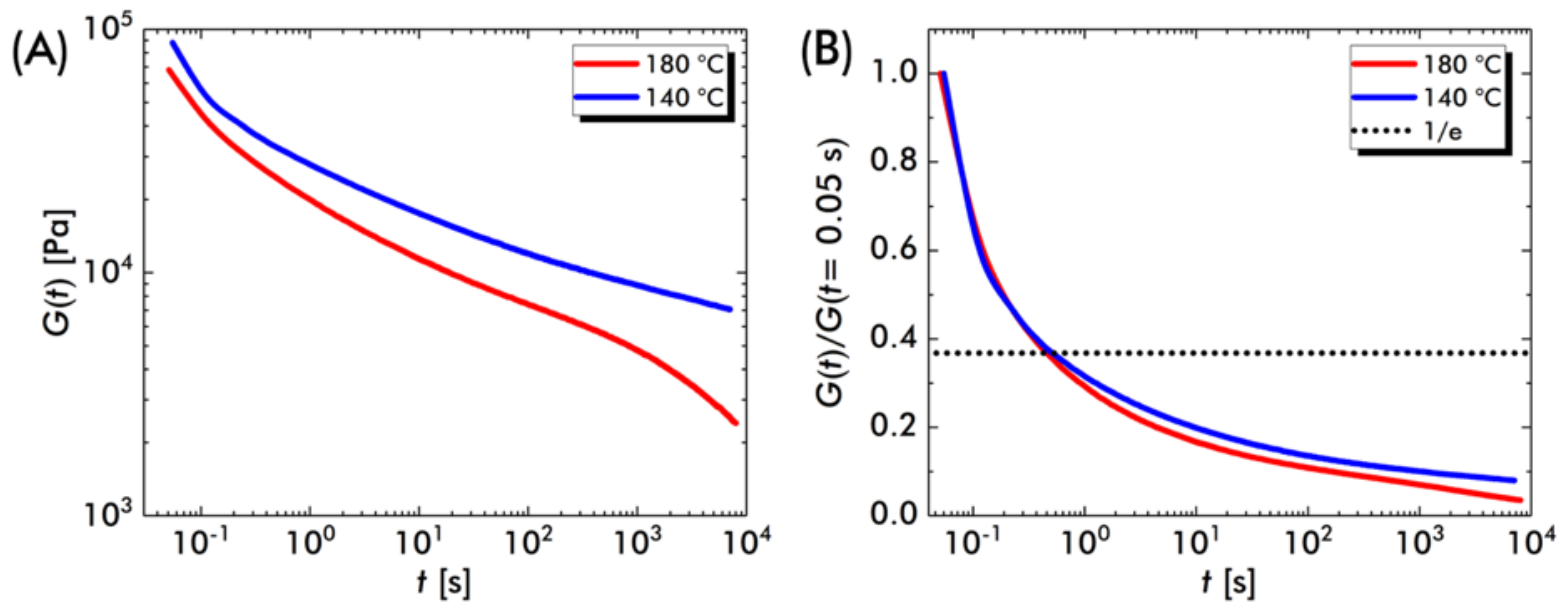

Figure 9. Comparison of PE- $v$ stress relaxation data presented in (A) log-log and (B) normalized semi-log formats. The black dashed line in (B) marks when the data decay to a value of $1 / e$. Data in both panels are the same as those presented in Figure 5B. Panel (B) could suggest that the relaxation time is $\sim 0.5 \mathrm{~s}$. This is, however, a gross underestimation, as clearly visible in panel (A). For instance, at $140{ }^{\circ} \mathrm{C}$ the stress is not relaxed, even after $8 \mathrm{~h}$.

Rather than using stress relaxation to solely evaluate vitrimer linear viscoelasticity, a better practice is to employ it in conjunction with other small strain experiments. Creep measurements, in particular, are useful for characterizing materials with very slow macroscopic dynamics. ${ }^{95}$ Rheological features observed in stress relaxation (i.e., plateaus and decays) are also observed in creep. In contrast to stress relaxation, however, creep experiments involve constant applied stress and torque, so the data do not become noisier over time and are not affected by the minimum torque limit. We have shown here that pairing creep compliance and recovery experiments allows the elastic and viscous components of the flow behavior (described by $J_{e}^{0}$ and $\eta_{0}$, respectively) to be deconvoluted. Evaluation of the $J(t)$ power law scaling also provides a model-free way to judge if the material has reached terminal relaxation. Compared with stress relaxation, creep has only been performed in a few vitrimer-related studies, ${ }^{1,2,6,14,26,28,30}$ but this technique serves as an efficient method to measure $\tau$, provided that terminal relaxation is attained. Furthermore, creep provides a direct method for showing if a material has achieved terminal relaxation or not (as was seen in this study). Similar to stress relaxation data, plotting $J(t)$ versus $t$ data on logarithmic axes enables accurate evaluation of the power law scaling at long times. SAOS experiments also could 
be illuminating with regards to vitrimer rheology. Commonly used in the study of other polymeric materials, SAOS may reveal rheological fingerprints that occur at high $\omega$ (i.e., short time scales). ${ }^{73,94}$ Creep compliance data may even be converted to the dynamic moduli to extend the frequency range (see Figure S43). In the case of the PE vitrimer in this study, however, the polydispersity of the neat $\mathrm{PE}$ and the presence of heterogeneities at multiple length scales makes it challenging to propose molecular models to rigorously interpret the SAOS measurements.

2) Self-assembly/associative cross-linking interplay dictates PE vitrimer rheology. According to previous work, ${ }^{56}$ graft-functionalized PE and PE vitrimer display similar types of morphology. Both macrophase separate into graft-rich and graft-poor domains. Within the graft-rich regions, the grafts assemble into aggregates that further arrange into a mass fractal. The length scales associated with the aggregate and fractal sizes were comparable between both materials; the presence of the cross-linker in PE vitrimer had a marginal effect on the structure. ${ }^{56}$ Despite the similarities in morphology, the PE- $g$ and PE- $v$ samples in this study had very different linear viscoelastic behaviors (Figure 10), suggesting that the interplay between self-assembly and associative cross-linking ultimately controls PE vitrimer rheology.
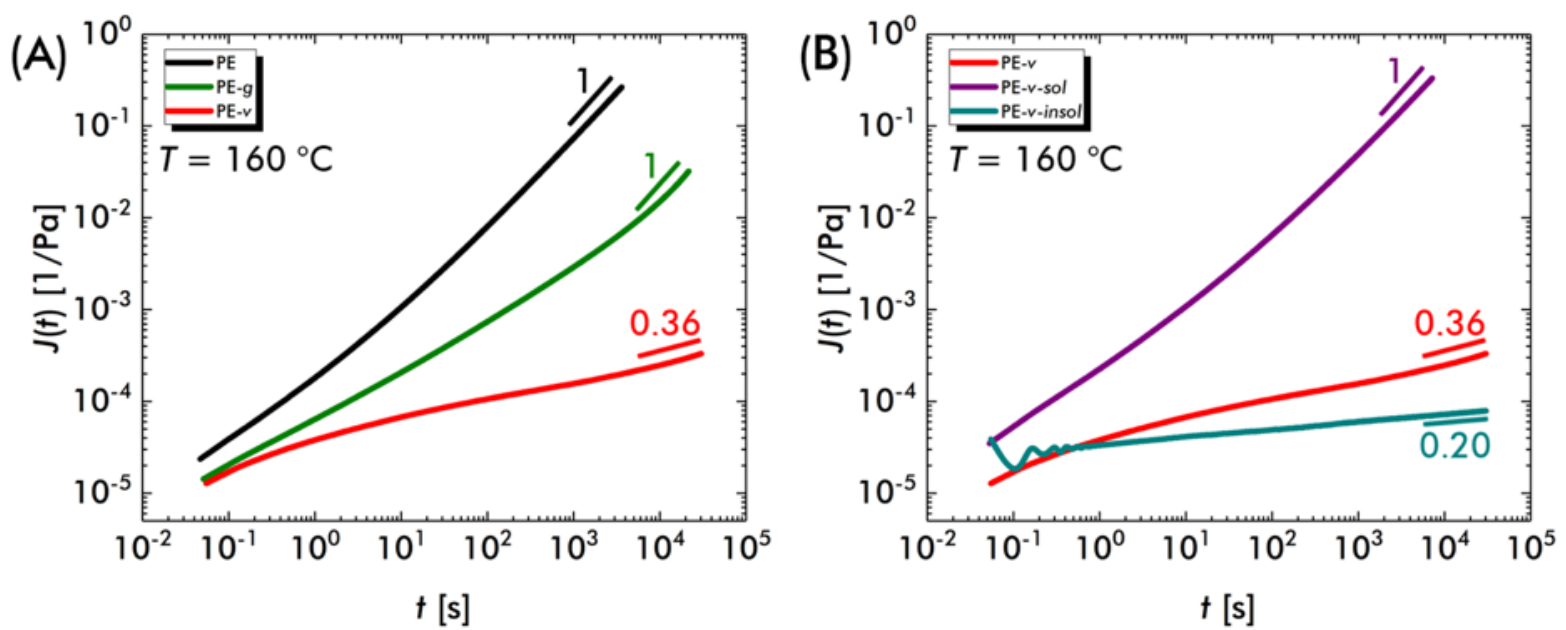

Figure 10. PE- $v$ creep compliance data at $160^{\circ} \mathrm{C}$ are compared to (A) PE, PE- $g$, (B) PE- $v$-sol, and PE- $v$-insol. The data are the same as those seen in Figures 4D, 5C, 6C, 7A, and 8B. 
PE- $g$ can be viewed to behave as a macrophase separated associative polymer in which each dioxaborolane maleimide graft acts as a "sticker" unit. Association is likely driven by polarity contrast between the boronic ester graft and the PE backbone. The PE backbone between each sticker connects two grafts within a single aggregate (forming a loop) or grafts that sit in different aggregates (forming a bridge). Both loops and bridges can increase the modulus. ${ }^{100}$ For systems of associating polymers that form large aggregates (i.e., much more than 2 stickers in an aggregate), Semenov and Rubinstein described two mechanisms that affect the stress relaxation. The first mechanism involves the diffusion of individual polymer chains (Figure 11A). For a single chain to diffuse, some of its stickers (but not necessarily all) must first disengage from their original aggregates and join with other aggregates. After this elementary event, segments of the chain relax via Rouse and reptation modes. The second mechanism concerns the diffusion of entire aggregates. For closely packed amorphous systems of aggregates, an imposed deformation perturbs the equilibrium structure. To relieve this deformation, the aggregates may reorganize via a hopping event. The energy barrier for hopping is very high, so this mechanism would be slower than chain diffusion. ${ }^{100}$ In the case of PE- $g$, because of the presence of phase separation of graftrich and graft-poor phases, the situation is expected to be even more complex. For our system, the molar concentration of grafts is only $\sim 0.3 \mathrm{~mol} \%$, and the volume fraction of the aggregates is quite dilute. Yet, it seems that graft disengagement and stress relaxation by rearrangement of phase separated regions and aggregate assemblies slows down stress relaxation considerably. At high temperatures, it takes less than $8 \mathrm{~h}$ to reach a steady-state flow. When PE-g is only heated to 140 ${ }^{\circ} \mathrm{C}$, however, it does not reach terminal relaxation within $20 \mathrm{~h}$. 


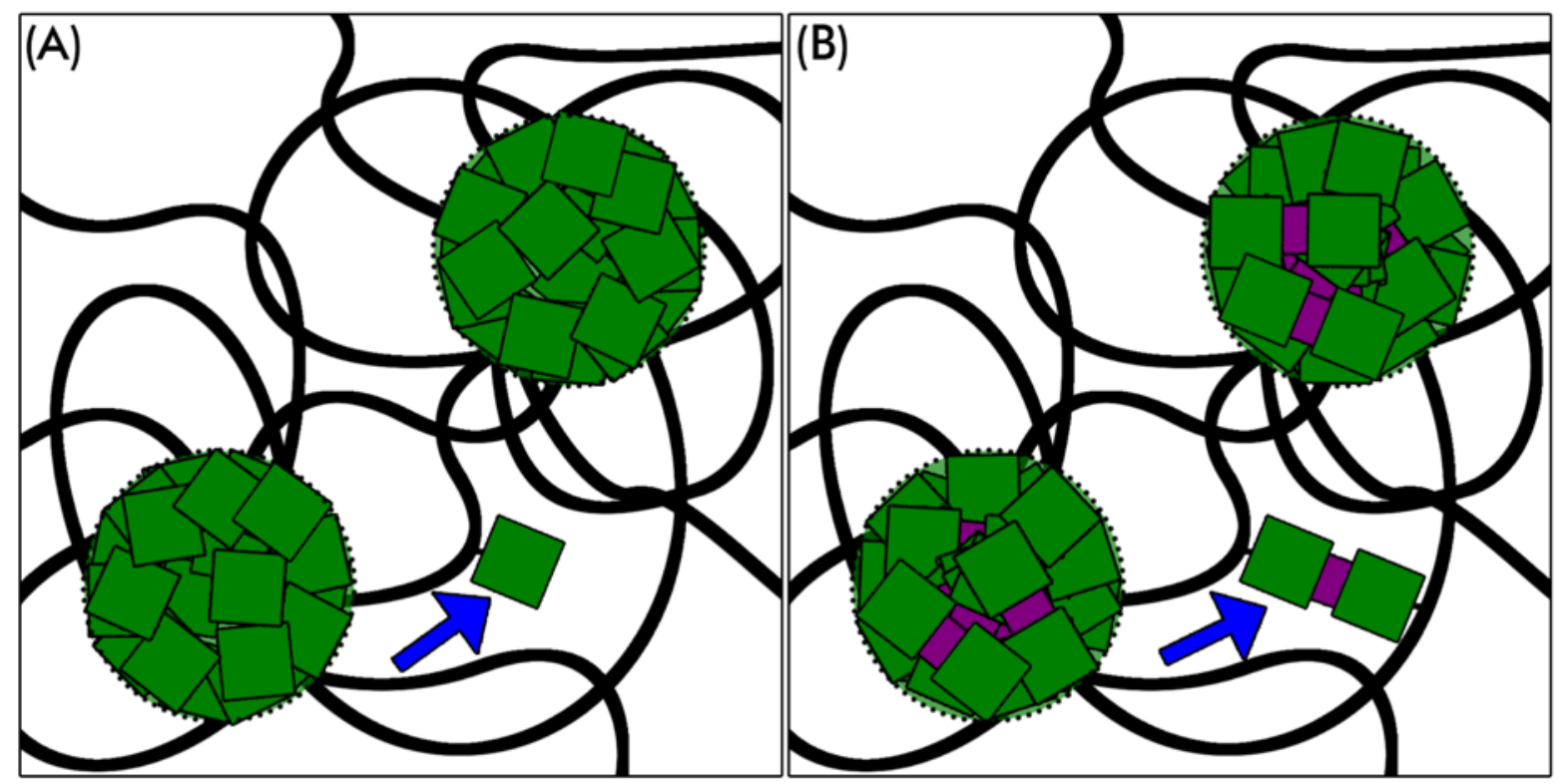

Figure 11. Illustration of proposed aggregate nanostructure in (A) PE-g and (B) PE- $v$. The green squares are grafts, the purple connectors are cross-links, and the black lines are PE strands between grafts. The blue arrow in each panel points to a graft or a pair of grafts connected by a cross-linker that have disengaged from an aggregate.

In homogeneous vitrimer systems that are well above the glass transition temperature, exchange of the cross-links is thought to be the rate-limiting step for terminal relaxation. ${ }^{2,54,55}$ For self-assembled vitrimers, however, that is likely not the case. PE- $v$ clearly does not exhibit the exponential or stretched exponential behavior anticipated for vitrimer materials. As discussed above, even in the absence of cross-links, the very presence of dioxaborolane maleimide grafts leads to aggregate formation and phase separation that greatly affect system dynamics and flow. Rheology experiments, whether under imposed stress or strain, show that PE vitrimers exhibit sluggish dynamics relative to PE-g and suggest that addition of the associative cross-linker alters the relaxation mechanisms of the insoluble graft-rich domains. Cross-link exchange between grafts should most likely occur within the same aggregate, where the cross-linker concentration is much higher than it is in the PE matrix. Such an exchange would not significantly reduce the topological stress in the system. ${ }^{101}$ Furthermore, due to the covalent linkages between individual dioxaborolane units, the graft disengagement event requires the pull out of two grafts that are 
connected by cross-linker (Figure 11B). This constraint doubles the activation energy of the elementary step and hinders subsequent relaxation of the PE chain segments.

Related to how microphase separation of PE- $v$-insol prevents it from fully relaxing over a reasonable time scale, the structure of PE- $v$-sol controls its linear viscoelasticity. This phase of the vitrimer, whose cross-link density is below the gel point, likely contains the lower molecular weight fraction of chains of the original PE. The SAOS data depicts this difference in the molecular weight distribution (Figure S38). At high $\omega$, both $G^{\prime}$ and $G^{\prime \prime}$ for PE-v-sol are lower than the values for neat PE, implying that it exhibits fewer Rouse and/or reptation modes. Even though its graft/ethylene molar ratio is low, the relatively high $\Delta E_{a c t}$ of PE- $v$-sol suggests the grafts are associated in some manner - either as cross-linked pairs or multi-graft aggregates. Furthermore, the high $J_{e}^{0}$ of PE- $v$-sol (and similarity to the values for the PE $+0.05 \mathrm{wt} \% \mathrm{DCP}$ sample) insinuates that this phase may contain nondynamically cross-linked PE chains.

Linear rheology experiments on blends containing insoluble portion of vitrimers nicely confirm that both hindering of cross-link disengagement and reorganization of cross-link (aggregate) rich and poor phase separated domains play essential roles in stress relaxation and creep of PE/dioxaborolane vitrimers.

3) PE-v blend rheology. The rheology of PE vitrimer will not only depend on the viscoelastic properties of the individual domains, but also on how these domains cooperate during relaxation. To get a sense of how the soluble and insoluble portions interact, we characterized the rheology of two different blends that each contained a minority fraction of PE- $v$-insol.

Blend PE/PE-v-insol, composed of a majority of neat polymer, serves as an example of a homopolymer/vitrimer composite. At $\omega>10 \mathrm{rad} / \mathrm{s}$ in SAOS, the dynamic moduli are similar to the values obtained for PE- $v$ (Figure S31). At lower $\omega$, however, the blend PE/PE- $v$-insol moduli begin to relax much faster. The hastened dynamics are further demonstrated in stress relaxation (Figures 12A and S32). Within $100 \mathrm{~s}$ at all temperatures, the relaxation modulus of the blend decreases by almost three decades. After $100 \mathrm{~s}$, the rate of relaxation is sensitive to the temperature. 
For $T=180^{\circ} \mathrm{C}, G(t)$ follows power law behavior, but as the temperature is decreased it plateaus. Creep measurements show that blend PE/PE- $v$-insol did not achieve steady-state behavior within $8 \mathrm{~h}$ (Figure 12B and S33). The creep compliance data display power law scalings between $0.30-$ 0.34, comparable to the PE- $v$ scalings.
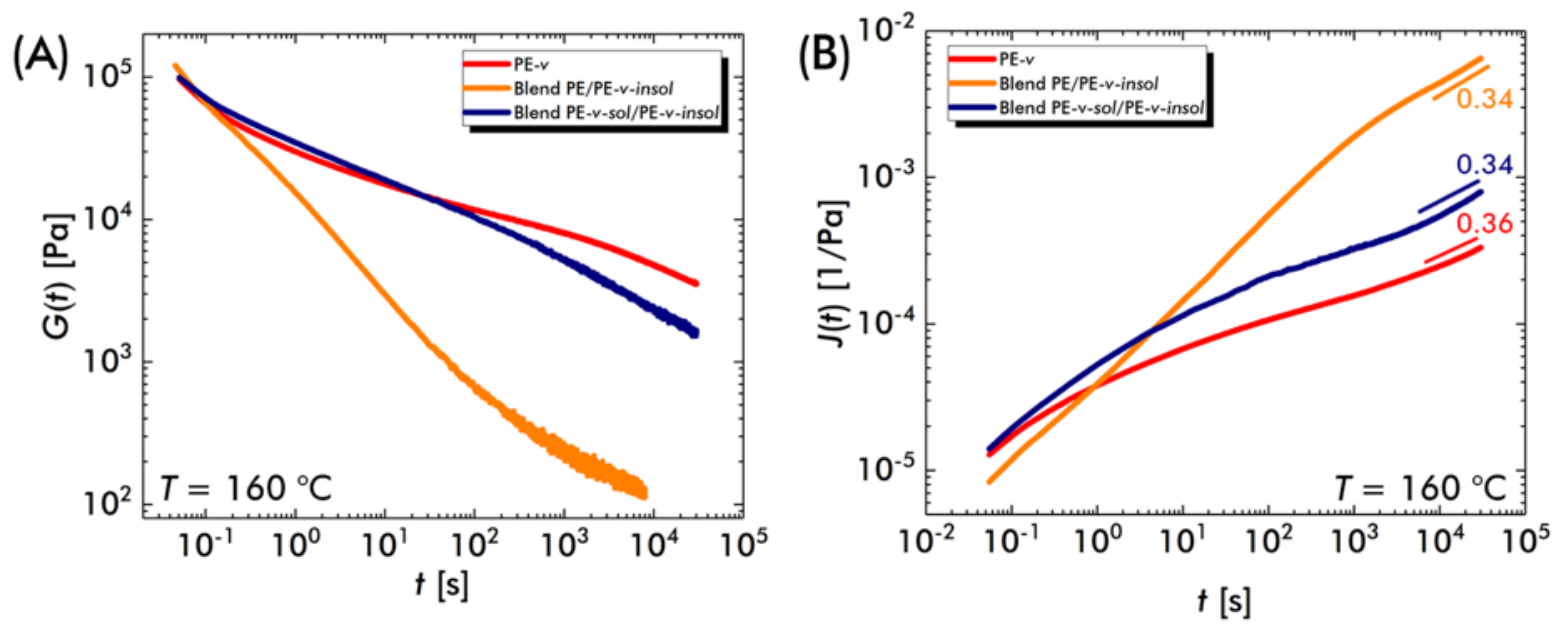

Figure 12. (A) Stress relaxation and (B) creep compliance data for PE- $v$, blend PE/PE-v-insol, and blend PE-v-sol/PE-v-insol.

Blend PE- $v$-sol/PE- $v$-insol, where the majority phase contained a dilute concentration of dioxaborolane maleimide, can be considered as a reconstruction of the original PE vitrimer. Accordingly, at all temperatures this blend exhibits rheological properties that were comparable to PE- $v$. The dynamic moduli measured during SAOS match the PE- $v$ values (Figure S34 and S40). $G^{\prime}$ is larger than $G^{\prime \prime}$ over the entire $\omega$ range. During stress relaxation (Figures 12A, S35, and S41A), the blend PE- $v$-sol/PE- $v$-insol $G(t)$ overlaps the PE- $v$ data at $t<100 \mathrm{~s}$, but decays faster than the original vitrimer at longer times. This difference could result from different interface structures of the macrophase separated regions. Indeed, few dioxaborolane grafts present in PE- $v$-sol could interact with PE- $v$-insol at the interface. The trends observed in the blend creep compliance curves are also consistent with the PE- $v$ behavior (Figure 12B and S41B). At $T=140$ and $160{ }^{\circ} \mathrm{C}$, the 
blend achieves the same power law scalings at long times as the original vitrimer. At $180{ }^{\circ} \mathrm{C}$, however, it expressed a scaling of 0.62 , higher than the PE- $v$ scaling at that temperature.

The findings of the blend studies suggest that the relaxation of PE- $v$ involves several dynamic processes working in concert. The graft-poor phase lubricates the graft-rich network and enables it to partially flow. However, in both blends (and presumably PE-v), the sluggish dynamics of the insoluble phase prevent the materials from reaching terminal relaxation. These findings also reveal that there is a synergistic interaction between the two phases. Blends PE/PE- $v$-insol and PE$v$-sol/PE- $v$-insol have dissimilar flow behaviors. This difference, however, is not solely due to viscoelastic contrast since $\mathrm{PE}$ and PE- $v$-sol have comparable rheological responses. The higher elasticity of blend PE- $v$-sol/PE- $v$-insol could arise from PE chain segments bridging graft aggregates across the interface between the two phases. Specifically, grafts from chains in the PE$v$-sol domain can traverse the interface and join aggregates in the PE- $v$-insol domain. As a result, the chains that span the interface would connect the two domains, just like compatibilizers do in polymer blends. ${ }^{102,103}$ When placed under small strain, the chain bridges would need to revert into loops to relieve stress, thereby slowing down relaxation.

4) Behavior of PE vitrimer during processing. Characterization of $\mathrm{PE}$ vitrimer materials reveal that the combination of self-assembly and associative cross-linking delays terminal relaxation. At first glance, this finding seems to underestimate the malleability of PE vitrimer. All the samples in this study were able to be extruded and compression molded into discs, so clearly, they are processable. The disconnect between the sluggish dynamics of PE vitrimer at small strains versus their ability to be processed points towards the importance of studying the nonlinear rheology. While the scope of this paper focuses on linear viscoelasticity, we will share a small set qualitative studies on nonlinear behavior to guide future investigations.

Figure 13 compares the raw strain and creep compliance of PE- $v$ under different shear stresses $(\sigma)$ at $T=140{ }^{\circ} \mathrm{C}$. At $\sigma=500 \mathrm{~Pa}$, the sample creeps slowly and only achieves a power law scaling of 0.22 after $8 \mathrm{~h}$. After a recovery period of $2 \mathrm{~h}, \sigma=10000 \mathrm{~Pa}$ was applied. For $t \leq$ 
$900 \mathrm{~s}, J(t)$ perfectly superimposes the data at lower stress. This overlap suggests that even at higher $\sigma$, the creep measurement was still within the linear regime. However, after $t=900 \mathrm{~s}$, when the material reaches a strain of $\sim 120 \%, J(t)$ begins to rapidly increase. The strain of $120 \%$ is coincident with the critical value seen in the strain sweep (Figure S2D). When $t=1800 \mathrm{~s}$, the stress was released, and the convection oven was lifted to reveal the sample fractured. Melt fracture occurred when the experiment was repeated. This melt fracture phenomenon is also captured in the strain sweep measurements (Figures S2 \& S3). Once the critical strain is reached, the complex moduli of PE- $v$, PE- $v$-insol, and blend PE- $v$-sol/PE- $v$-insol sharply decrease, which contrasted the gradual decrease expressed by PE and PE-g. These results are also consistent with the melt-flow index measurements performed by Röttger et al. ${ }^{21}$ At an applied load of $2.16 \mathrm{~kg}$, PE vitrimer did not flow within the 10 min measurement period. When the load was increased to $10 \mathrm{~kg}$ (i.e., higher shear stress), the material flowed. ${ }^{21}$ Based on these previous and current observations, we suspect that the processability of PE vitrimer stems not from its viscoelastic properties, but rather its ability to repeatedly fracture and heal. This flow mechanism is similar to how dough passes through a pasta extruder.
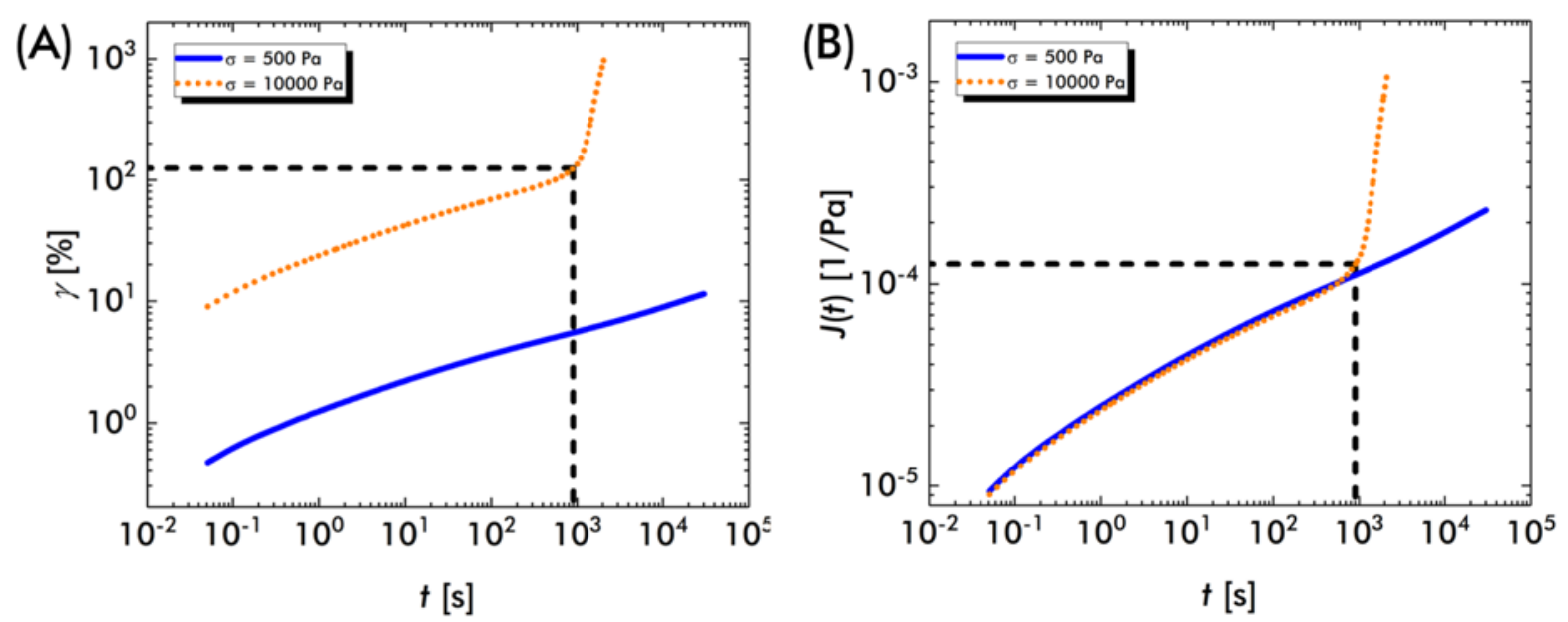

Figure 13. (A) Raw strain $(\gamma)$ and (B) creep compliance curves of PE- $v$ at different $\sigma$. The black dashed lines correspond to $t=900 \mathrm{~s}$. The $\sigma=500 \mathrm{~Pa}$ data are the same as seen in Figure 6C. 
This concept was further explored by comparing the extrudability of PE- $v$ and PE- $v$-insol. Each material was loaded into the DSM extruder described previously (screw speed $=100 \mathrm{rpm}$ and $T=170{ }^{\circ} \mathrm{C}$ ), mixed for $8 \mathrm{~min}$, and then released. The PE- $v$ extrudate emerged as a smooth plug, while the PE-v-insol extrudate was lumpy with cracks on the surface (Figure 14). The difference in extrudability advocates the importance of the graft-poor phase for facilitating processing. Not only does this phase effectively reduce the friction between the insoluble domains, but it possibly acts as a binding agent that promotes healing of defects in the material.

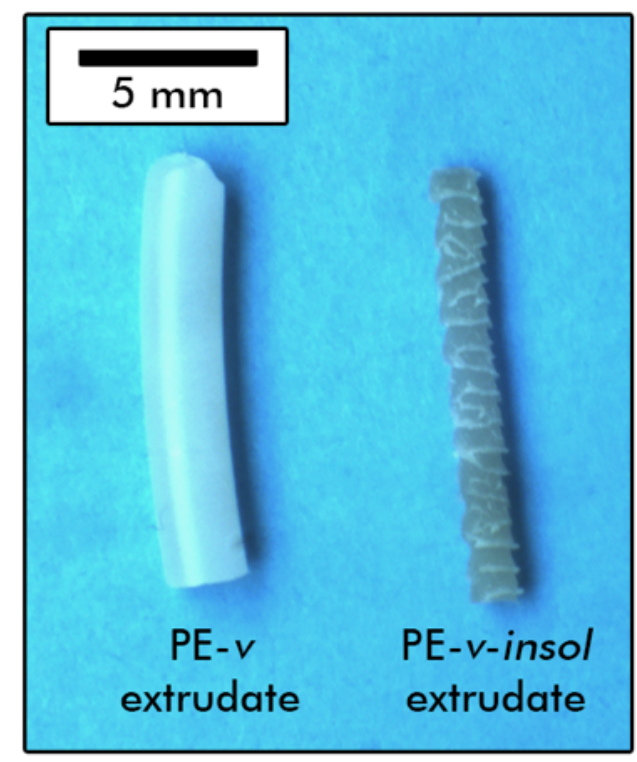

Figure 14. Photo of PE- $v$ and PE-v-insol extrudates.

\section{Conclusions}

We investigated the linear viscoelasticity and flow behavior of PE/dioxaborolane vitrimer materials. For noncross-linked graft functionalized PE, macrophase separation and graft aggregation decelerate the polymer chain dynamics. For PE vitrimer, the addition of the associative cross-linker effectively prevents terminal relaxation. The rheology of the PE vitrimer reflects the distinct graft-rich and graft-poor phases that comprise the material. The insoluble graft-rich network barely relaxes under small strain; its dynamics are likely hindered by the high energy 
barrier for graft disengagement from an aggregate. Conversely, the soluble graft-poor phase acts as both a lubricant and binding agent for the insoluble phase. Interactions between the two phases can also enhance the elastic response of the vitrimer.

PE vitrimer does not flow readily when a small strain is imposed, but easily flows when it is subject to high stress. The high stress causes PE vitrimer melt to fracture, but this fracture can heal. By repeatedly fracturing and healing, the material can flow. Such mechanism is particularly efficient when there is a lubricating phase. In such conditions, extrusion of PE vitrimer produces a plug with a smooth surface. In the absence of a lubricating phase, the plug has a corrugated surface and is an assembly of fractured pieces partially glued together (such as in the case of the isolated insoluble portion of PE vitrimer).

While additional studies and better-defined model systems are needed to fully resolve the relationship between the vitrimer morphology and linear viscoelasticity, these findings make it clear that both associative cross-linking and self-assembly significantly impact the rheology. The interplay between these factors is only becoming more important as the vitrimer concept continues to expand to different types of polymer backbones and architectures. ${ }^{54,55}$ Furthermore, selfassembly offers a powerful approach for enhancing vitrimer properties. The ability to reversibly trigger microphase separation could be utilized to create solvent-resistant materials with adaptive nanostructures, which are needed for next-generation electrolytes, separations membranes, and biomaterials. ${ }^{104}$ Macrophase separation could be harnessed to aid processing and self-healing. Vitrimers could even be used as dopants for enhancing the melt properties of commodity polymers. Before these applications can be realized, however, a deeper understanding of vitrimer dynamics needs to be elucidated. To achieve this, multiple rheological experiments and particularly nonrheology studies are needed to rigorously clarify vitrimer structure-dynamics relationships.

\section{Author information}

Corresponding Authors

*E-mail: ralm.ricarte@espci.fr and ludwik.leibler@espci.fr 
ORCID

Ralm Gerick Ricarte: 0000-0003-1018-6083

François Tournilhac: 0000-0002-6775-1584

Michel Cloître: 0000-0002-0869-6730

Notes

The authors have no competing financial interest.

\section{Acknowledgements}

We are very grateful to Mickaël Pomes-Hadda, Sarah Goujard, and Quentin Nozet for helping us perform the rheological measurements. We would also like to acknowledge helpful discussions with Julie Kornfield, Sanat Kumar, and Ralph Colby. The research leading to these results has received funding from the People Programme (Marie Curie Actions) of the European Union's Seventh Framework Programme (FP7/2007-2013) under REA grant agreement n. PCOFUNDGA-2013-609102, through the PRESTIGE programme (2017-2-0012) coordinated by Campus France.

\section{Supporting information}

Fourier transform infrared spectra of PE, PE $+0.05 \mathrm{wt} \%$ DCP, PE-g, and blends, strain sweeps, supplementary rheology data for all samples, influence of thermal degradation on PE- $g$ and blend PE-v-sol/PE-v-insol, comparison of PE, PE-g, PE- $v$, PE- $v$-sol, and PE-v-insol rheology, comparison of PE- $v$ and PE- $v$-sol/PE- $v$-insol blend rheology, conversion of creep compliance to dynamic moduli, and estimation of cross-link densities are provided in the Supporting Information. 


\section{References}

${ }^{1}$ Montarnal, D.; Capelot, M.; Tournilhac, F.; Leibler, L. Silica-Like Malleable Materials from Permanent Organic Networks. Science 2011, 334, 965-968.

${ }^{2}$ Capelot, M.; Unterlass, M. M.; Tournilhac, F.; Leibler, L. Catalytic Control of the Vitrimer Glass Transition. ACS Macro Lett. 2012, 1, 789-792.

${ }^{3}$ Capelot, M.; Montarnal, D.; Tournilhac, F.; Leibler, L. Metal-Catalyzed Transesterification for Healing and Assembling of Thermosets. J. Am. Chem. Soc. 2012, 134, 7664-7667.

${ }^{4}$ Brutman, J. P.; Delgado, P. A.; Hillmyer, M. A. Polylactide Vitrimers. ACS Macro Lett. 2014, 3, $607-610$.

${ }^{5}$ Fortman, D. J.; Brutman, J. P.; Cramer, C. J.; Hillmyer, M. A.; Dichtel, W. R. Mechanically Activated, Catalyst-Free Polyhydroxyurethane Vitrimers. J. Am. Chem. Soc. 2015, 137, 1401914022 .

${ }^{6}$ Denissen, W.; Rivero, G.; Nicolaÿ, R.; Leibler, L.; Winne, J. M.; Du Prez, F. E. Vinylogous Urethane Vitrimers. Adv. Funct. Mater. 2015, 25, 2451-2457.

${ }^{7}$ Ma, Z.; Wang, Y.; Zhu, J.; Yu, J.; Hu, Z. Bio-Based Epoxy Vitrimers: Reprocessibility, Controllable Shape Memory, and Degradability. J. Polym. Sci. Part A Polym. Chem. 2017, 55, 1790-1799.

${ }^{8}$ Stukenbroeker, T.; Wang, W.; Winne, J. M.; Du Prez, F. E.; Nicolaÿ, R.; Leibler, L. Polydimethylsiloxane Quenchable Vitrimers. Polym. Chem. 2017, 8, 6590-6593.

${ }^{9}$ Demongeot, A.; Groote, R.; Goossens, H.; Hoeks, T.; Tournilhac, F.; Leibler, L. Cross-Linking of Poly(Butylene Terephthalate) by Reactive Extrusion Using Zn(II) Epoxy-Vitrimer Chemistry. Macromolecules 2017, 50, 6117-6127.

${ }^{10}$ Zhou, Y.; Goossens, J. G. P.; Sijbesma, R. P.; Heuts, J. P. A. Poly(Butylene Terephthalate)/Glycerol-Based Vitrimers via Solid-State Polymerization. Macromolecules 2017, $50,6742-6751$. 
${ }^{11}$ Geng, H.; Wang, Y.; Yu, Q.; Gu, S.; Zhou, Y.; Xu, W.; Zhang, X.; Ye, D. Vanillin-Based Polyschiff Vitrimers: Reprocessability and Chemical Recyclability. ACS Sustain. Chem. Eng. 2018, 6, 15463-15470.

${ }^{12}$ Guerre, M.; Taplan, C.; Nicolaÿ, R.; Winne, J. M.; Du Prez, F. E. Fluorinated Vitrimer Elastomers with a Dual Temperature Response. J. Am. Chem. Soc. 2018, 140, 13272-13284.

${ }^{13}$ Zhou, Y.; Goossens, J. G. P.; van den Bergen, S.; Sijbesma, R. P.; Heuts, J. P. A. In Situ Network Formation in PBT Vitrimers via Processing-Induced Deprotection Chemistry. Macromol. Rapid Commun. 2018, 39, 1800356.

${ }^{14}$ Breuillac, A.; Kassalias, A.; Nicolaÿ, R. Polybutadiene Vitrimers Based on Dioxaborolane Chemistry and Dual Networks with Static and Dynamic Cross-Links. Macromolecules 2019, 52, $7102-7113$.

${ }^{15}$ Caffy, F.; Nicolaÿ, R. Transformation of Polyethylene into a Vitrimer by Nitroxide Radical Coupling of a Bis-Dioxaborolane. Polym. Chem. 2019, 10, 3107-3115.

${ }^{16}$ Scott, T. F.; Schneider, A. D.; Cook, W. D.; Bowman, C. N. Photoinduced Plasticity in CrossLinked Polymers. Science 2005, 308, 1615-1617.

${ }^{17}$ Lu, Y.; Tournilhac, F.; Leibler, L.; Guan, Z. Making Insoluble Polymer Networks Malleable via Olefin Metathesis. J. Am. Chem. Soc. 2012, 134, 8424-8427.

${ }^{18}$ Taynton, P.; Yu, K.; Shoemaker, R. K.; Jin, Y.; Qi, H. J.; Zhang, W. Heat- or Water-Driven Malleability in a Highly Recyclable Covalent Network Polymer. Adv. Mater. 2014, 26, 39383942.

${ }^{19}$ Cromwell, O. R.; Chung, J.; Guan, Z. Malleable and Self-Healing Covalent Polymer Networks through Tunable Dynamic Boronic Ester Bonds. J. Am. Chem. Soc. 2015, 137, 6492-6495.

${ }^{20}$ Cash, J. J.; Kubo, T.; Bapat, A. P.; Sumerlin, B. S. Room-Temperature Self-Healing Polymers Based on Dynamic-Covalent Boronic Esters. Macromolecules 2015, 48, 2098-2106. 
${ }^{21}$ Röttger, M.; Domenech, T.; van der Weegen, R.; Breuillac, A.; Nicolaÿ, R.; Leibler, L. HighPerformance Vitrimers from Commodity Thermoplastics through Dioxaborolane Metathesis. Science 2017, 356, 62-65.

${ }^{22}$ Snyder, R. L.; Fortman, D. J.; De Hoe, G. X.; Hillmyer, M. A.; Dichtel, W. R. Reprocessable Acid-Degradable Polycarbonate Vitrimers. Macromolecules 2018, 51, 389-397.

${ }^{23}$ Ishibashi, J. S. A.; Kalow, J. A. Vitrimeric Silicone Elastomers Enabled by Dynamic Meldrum's Acid-Derived Cross-Links. ACS Macro Lett. 2018, 7, 482-486.

${ }^{24}$ He, C.; Shi, S.; Wang, D.; Helms, B. A.; Russell, T. P. Poly(Oxime-Ester) Vitrimers with Catalyst-Free Bond Exchange. J. Am. Chem. Soc. 2019, 141, 13753-13757.

${ }^{25}$ Niu, X.; Wang, F.; Li, X.; Zhang, R.; Wu, Q.; Sun, P. Using $\mathrm{Zn}^{2+}$ Ionomer To Catalyze Transesterification Reaction in Epoxy Vitrimer. Ind. Eng. Chem. Res. 2019, 58, 5698-5706.

${ }^{26}$ Tretbar, C. A.; Neal, J. A.; Guan, Z. Direct Silyl Ether Metathesis for Vitrimers with Exceptional Thermal Stability. J. Am. Chem. Soc. 2019, 141, 16595-16599.

${ }^{27}$ Tellers, J.; Pinalli, R.; Soliman, M.; Vachon, J.; Dalcanale, E. Reprocessable Vinylogous Urethane Cross-Linked Polyethylene via Reactive Extrusion. Polym. Chem. 2019, 48-50.

${ }^{28}$ Yang, Y.; Pei, Z.; Zhang, X.; Tao, L.; Wei, Y.; Ji, Y. Carbon Nanotube-Vitrimer Composite for Facile and Efficient Photo-Welding of Epoxy. Chem. Sci. 2014, 5, 3486-3492.

${ }^{29}$ Chabert, E.; Vial, J.; Cauchois, J.-P.; Mihaluta, M.; Tournilhac, F. Multiple Welding of Long Fiber Epoxy Vitrimer Composites. Soft Matter 2016, 12, 4838-4845.

${ }^{30} \mathrm{Li}$, L.; Chen, X.; Jin, K.; Torkelson, J. M. Vitrimers Designed Both To Strongly Suppress Creep and To Recover Original Cross-Link Density after Reprocessing: Quantitative Theory and Experiments. Macromolecules 2018, 51, 5537-5546.

${ }^{31}$ Han, H.; Xu, X. Poly(Methyl Methacrylate)-Epoxy Vitrimer Composites. J. Appl. Polym. Sci. 2018, 135, 46307. 
${ }^{32}$ Denissen, W.; De Baere, I.; Van Paepegem, W.; Leibler, L.; Winne, J.; Du Prez, F. E. Vinylogous Urea Vitrimers and Their Application in Fiber Reinforced Composites. Macromolecules 2018, 51, 2054-2064.

${ }^{33}$ Yan, P.; Zhao, W.; Jiang, L.; Wu, B.; Hu, K.; Yuan, Y.; Lei, J. Reconfiguration and Shape Memory Triggered by Heat and Light of Carbon Nanotube-Polyurethane Vitrimer Composites. $J$. Appl. Polym. Sci. 2018, 135, 45784.

${ }^{34}$ Meng, F.; Saed, M. O.; Terentjev, E. M. Elasticity and Relaxation in Full and Partial Vitrimer Networks. Macromolecules 2019, 52, 7423-7429.

${ }^{35}$ Saed, M. O.; Gablier, A.; Terentjev, E. M. Liquid Crystalline Vitrimers with Full or Partial Boronic-Ester Bond Exchange. Adv. Funct. Mater. 2019, 1906458, 1906458.

${ }^{36}$ Zhou, Y.; Groote, R.; Goossens, J. G. P.; Sijbesma, R. P.; Heuts, J. P. A. Tuning PBT Vitrimer Properties by Controlling the Dynamics of the Adaptable Network. Polym. Chem. 2019, 10, 136144.

${ }^{37}$ Erice, A.; Azcune, I.; Ruiz de Luzuriaga, A.; Ruipérez, F.; Irigoyen, M.; Matxain, J. M.; Asua, J. M.; Grande, H.; Rekondo, A. Effect of Regioisomerism on Processability and Mechanical Properties of Amine/Urea Exchange Based Poly(Urea-Urethane) Vitrimers. ACS Appl. Polym. Mater. 2019, 1, 2472-2481.

${ }^{38}$ Zhao, S.; Abu-Omar, M. M. Catechol-Mediated Glycidylation toward Epoxy Vitrimers/Polymers with Tunable Properties. Macromolecules 2019, 52, 3646-3654.

${ }^{39}$ Kloxin, C. J.; Scott, T. F.; Bowman, C. N. Stress Relaxation via Addition-Fragmentation Chain Transfer in a Thiol-Ene Photopolymerization. Macromolecules 2009, 42, 2551-2556.

${ }^{40}$ Denissen, W.; Droesbeke, M.; Nicolaÿ, R.; Leibler, L.; Winne, J. M.; Du Prez, F. E. Chemical Control of the Viscoelastic Properties of Vinylogous Urethane Vitrimers. Nat. Commun. 2017, 8, 14857. 
${ }^{41}$ Self, J. L.; Dolinski, N. D.; Zayas, M. S.; Read de Alaniz, J.; Bates, C. M. Brønsted-AcidCatalyzed Exchange in Polyester Dynamic Covalent Networks. ACS Macro Lett. 2018, 7, 817821.

${ }^{42}$ Chen, M.; Zhou, L.; Wu, Y.; Zhao, X.; Zhang, Y. Rapid Stress Relaxation and Moderate Temperature of Malleability Enabled by the Synergy of Disulfide Metathesis and Carboxylate Transesterification in Epoxy Vitrimers. ACS Macro Lett. 2019, 8, 255-260.

${ }^{43}$ Yang, Y.; Pei, Z.; Li, Z.; Wei, Y.; Ji, Y. Making and Remaking Dynamic 3D Structures by Shining Light on Flat Liquid Crystalline Vitrimer Films without a Mold. J. Am. Chem. Soc. 2016, 138, 2118-2121.

${ }^{44}$ Altuna, F. I.; Hoppe, C. E.; Williams, R. J. J. Shape Memory Epoxy Vitrimers Based on DGEBA Crosslinked with Dicarboxylic Acids and Their Blends with Citric Acid. RSC Adv. 2016, 6, 8864788655 .

${ }^{45}$ Pei, Z.; Yang, Y.; Chen, Q.; Wei, Y.; Ji, Y. Regional Shape Control of Strategically Assembled Multishape Memory Vitrimers. Adv. Mater. 2016, 28, 156-160.

${ }^{46}$ Ciarella, S.; Ellenbroek, W. Swap-Driven Self-Adhesion and Healing of Vitrimers. Coatings 2019, 9, 114.

${ }^{47}$ Lyon, G. B.; Cox, L. M.; Goodrich, J. T.; Baranek, A. D.; Ding, Y.; Bowman, C. N. Remoldable Thiol-Ene Vitrimers for Photopatterning and Nanoimprint Lithography. Macromolecules 2016, 49, 8905-8913.

${ }^{48}$ Shi, Q.; Yu, K.; Kuang, X.; Mu, X.; Dunn, C. K.; Dunn, M. L.; Wang, T.; Qi, H.J. Recyclable 3D Printing of Vitrimer Epoxy. Mater. Horizons 2017, 4, 598-607.

${ }^{49}$ Wang, Z.; Tian, H.; He, Q.; Cai, S. Reprogrammable, Reprocessible, and Self-Healable Liquid Crystal Elastomer with Exchangeable Disulfide Bonds. ACS Appl. Mater. Interfaces 2017, 9, $33119-33128$. 
${ }^{50}$ Cox, L. M.; Sun, X.; Wang, C.; Sowan, N.; Killgore, J. P.; Long, R.; Wu, H. A.; Bowman, C. N.; Ding, Y. Light-Stimulated Permanent Shape Reconfiguration in Cross-Linked Polymer Microparticles. ACS Appl. Mater. Interfaces 2017, 9, 14422-14428.

${ }^{51}$ Tran, T. N.; Rawstron, E.; Bourgeat-Lami, E.; Montarnal, D. Formation of Cross-Linked Films from Immiscible Precursors through Sintering of Vitrimer Nanoparticles. ACS Macro Lett. 2018, 7, 376-380.

${ }^{52}$ Kloxin, C. J.; Bowman, C. N. Covalent Adaptable Networks: Smart, Reconfigurable and Responsive Network Systems. Chem. Soc. Rev. 2013, 42, 7161-7173.

${ }^{53}$ Denissen, W.; Winne, J. M.; Du Prez, F. E. Vitrimers: Permanent Organic Networks with Glasslike Fluidity. Chem. Sci. 2016, 7, 30-38.

${ }^{54}$ Scheutz, G. M.; Lessard, J. J.; Sims, M. B.; Sumerlin, B. S. Adaptable Crosslinks in Polymeric Materials: Resolving the Intersection of Thermoplastics and Thermosets. J. Am. Chem. Soc. 2019, $141,16181-16196$.

${ }^{55}$ Winne, J. M.; Leibler, L.; Du Prez, F. E. Dynamic Covalent Chemistry in Polymer Networks: A Mechanistic Perspective. Polym. Chem. 2019, 10, 6091-6108.

${ }^{56}$ Ricarte, R. G.; Tournilhac, F.; Leibler, L. Phase Separation and Self-Assembly in Vitrimers: Hierarchical Morphology of Molten and Semicrystalline Polyethylene/Dioxaborolane Maleimide Systems. Macromolecules 2019, 52, 432-443.

${ }^{57}$ Chen, X.; Li, L.; Wei, T.; Torkelson, J. M. Reprocessable Polymer Networks Designed with Hydroxyurethane Dynamic Cross-links: Effect of Backbone Structure on Network Morphology, Phase Segregation, and Property Recovery. Macromol. Chem. Phys. 2019, 1900083, 1900083.

${ }^{58}$ Capelot, M. Chimie de Polycondensation, Polymères Supramoléculaires et Vitrimères. Ph.D. Dissertation, Université Pierre et Marie Curie, Paris, FR, 2013.

${ }^{59}$ Long, R.; Qi, H. J.; Dunn, M. L. Modeling the Mechanics of Covalently Adaptable Polymer Networks with Temperature-Dependent Bond Exchange Reactions. Soft Matter 2013, 9, 4083. 
${ }^{60}$ Ma, J.; Mu, X.; Bowman, C. N.; Sun, Y.; Dunn, M. L.; Qi, H. J.; Fang, D. A Photoviscoplastic Model for Photoactivated Covalent Adaptive Networks. J. Mech. Phys. Solids 2014, 70, 84-103. ${ }^{61}$ Yu, K.; Shi, Q.; Wang, T.; Dunn, M. L.; Jerry Qi, H. A Computational Model for Surface Welding in Covalent Adaptable Networks Using Finite-Element Analysis. J. Appl. Mech. 2016, $83,1-11$.

${ }^{62}$ Yang, H.; Yu, K.; Mu, X.; Wei, Y.; Guo, Y.; Qi, H. J. Molecular Dynamics Studying on Welding Behavior in Thermosetting Polymers Due to Bond Exchange Reactions. RSC Adv. 2016, 6, 2247622487.

${ }^{63}$ Yu, K.; Shi, Q.; Li, H.; Jabour, J.; Yang, H.; Dunn, M. L.; Wang, T.; Qi, H. J. Interfacial Welding of Dynamic Covalent Network Polymers. J. Mech. Phys. Solids 2016, 94, 1-17.

${ }^{64}$ Sun, X.; Wu, H.; Long, R. Thermomechanics of a Temperature Sensitive Covalent Adaptable Polymer with Bond Exchange Reactions. Soft Matter 2016, 12, 8847-8860.

${ }^{65}$ Vernerey, F. J.; Long, R.; Brighenti, R. A Statistically-Based Continuum Theory for Polymers with Transient Networks. J. Mech. Phys. Solids 2017, 107, 1-20.

${ }^{66}$ Pritchard, R. H.; Redmann, A.-L.; Pei, Z.; Ji, Y.; Terentjev, E. M. Vitrification and Plastic Flow in Transient Elastomer Networks. Polymer 2016, 95, 45-51.

${ }^{67}$ Luo, C.; Shi, X.; Lei, Z.; Zhu, C.; Zhang, W.; Yu, K. Effects of Bond Exchange Reactions and Relaxation of Polymer Chains on the Thermomechanical Behaviors of Covalent Adaptable Network Polymers. Polymer 2018, 153, 43-51.

${ }^{68}$ Meng, F.; Pritchard, R. H.; Terentjev, E. M. Stress Relaxation, Dynamics, and Plasticity of Transient Polymer Networks. Macromolecules 2016, 49, 2843-2852.

${ }^{69}$ Meng, F.; Terentjev, E. Transient Network at Large Deformations: Elastic-Plastic Transition and Necking Instability. Polymers 2016, 8, 108.

${ }^{70}$ Rovigatti, L.; Nava, G.; Bellini, T.; Sciortino, F. Self-Dynamics and Collective Swap-Driven Dynamics in a Particle Model for Vitrimers. Macromolecules 2018, 51, 1232-1241. 
${ }^{71}$ Smallenburg, F.; Leibler, L.; Sciortino, F. Patchy Particle Model for Vitrimers. Phys. Rev. Lett. 2013, 111, 188002.

${ }^{72}$ Ciarella, S.; Sciortino, F.; Ellenbroek, W. G. Dynamics of Vitrimers: Defects as a Highway to Stress Relaxation. Phys. Rev. Lett. 2018, 121, 058003.

${ }^{73}$ Snijkers, F.; Pasquino, R.; Maffezzoli, A. Curing and Viscoelasticity of Vitrimers. Soft Matter 2017, 13, 258-268.

${ }^{74}$ Stadler, R.; de Lucca Freitas, L. Thermoplastic Elastomers by Hydrogen Bonding 1. Rheological Properties of Modified Polybutadiene. Colloid Polym. Sci. 1986, 264, 773-778.

${ }^{75}$ Zhang, Z.; Chen, Q.; Colby, R. H. Dynamics of Associative Polymers. Soft Matter 2018, 14, 2961-2977.

${ }^{76}$ Ahmadi, M.; Jangizehi, A.; van Ruymbeke, E.; Seiffert, S. Deconvolution of the Effects of Binary Associations and Collective Assemblies on the Rheological Properties of Entangled SideChain Supramolecular Polymer Networks. Macromolecules 2019, 52, 5255-5267.

${ }^{77}$ Leibler, L.; Rubinstein, M.; Colby, R. H. Dynamics of Reversible Networks. Macromolecules 1991, 24, 4701-4707.

${ }^{78}$ Rubinstein, M.; Semenov, A. N. Dynamics of Entangled Solutions of Associating Polymers. Macromolecules 2001, 34, 1058-1068.

${ }^{79}$ Palierne, J. F. Linear Rheology of Viscoelastic Emulsions with Interfacial Tension. Rheol. Acta 1990, 29, $204-214$.

${ }^{80}$ Graebling, D.; Muller, R.; Palierne, J. F. Linear Viscoelastic Behavior of Some Incompatible Polymer Blends in the Melt. Interpretation of Data with a Model of Emulsion of Viscoelastic Liquids. Macromolecules 1993, 26, 320-329.

${ }^{81}$ Pötschke, P.; Paul, D. R. Formation of Co-Continuous Structures in Melt-Mixed Immiscible Polymer Blends. J. Macromol. Sci. Part C Polym. Rev. 2003, 43, 87-141. 
${ }^{82}$ Van Puyvelde, P.; Moldenaers, P. Rheology and Morphology Development in Immiscible Polymer Blends. In Rheology Reviews; John Wiley \& Sons, Inc: Hoboken, NJ, USA, 2005; pp. $101-145$.

${ }^{83}$ Alejandro, J. M.; Michell, R. M. Polymer Morphology; Guo, Q., Ed.; John Wiley \& Sons, Inc: Hoboken, NJ, USA, 2016.

${ }^{84}$ Chen, Q.; Tudryn, G. J.; Colby, R. H. Ionomer Dynamics and the Sticky Rouse Model. J. Rheol. 2013, 57, 1441-1462.

${ }^{85}$ Chen, Q.; Huang, C.; Weiss, R. A.; Colby, R. H. Viscoelasticity of Reversible Gelation for Ionomers. Macromolecules 2015, 48, 1221-1230.

${ }^{86}$ Fredrickson, G. H.; Bates, F. S. Dynamics of Block Copolymers: Theory and Experiment. Annu. Rev. Mater. Sci. 1996, 26, 501-550.

${ }^{87}$ Colby, R. H. Block Copolymer Dynamics. Curr. Opin. Colloid Interface Sci. 1996, 1, 454-465. ${ }^{88}$ Watanabe, H. Rheology of Diblock Copolymer Micellar Systems. Acta Polym. 1997, 48, $215-$ 233.

${ }^{89}$ Oparaji, O.; Narayanan, S.; Sandy, A.; Ramakrishnan, S.; Hallinan, D. Structural Dynamics of Strongly Segregated Block Copolymer Electrolytes. Macromolecules 2018, 51, 2591-2603.

${ }^{90}$ Lewis, R. M.; Beech, H. K.; Jackson, G. L.; Maher, M. J.; Kim, K.; Narayanan, S.; Lodge, T. P.; Mahanthappa, M. K.; Bates, F. S. Dynamics of a Supercooled Disordered Sphere-Forming Diblock Copolymer as Determined by X-Ray Photon Correlation and Dynamic Mechanical Spectroscopies. ACS Macro Lett. 2018, 7, 1486-1491.

${ }^{91}$ Semenov, A. N.; Joanny, J.-F.; Khokhlov, A. R. Associating Polymers: Equilibrium and Linear Viscoelasticity. Macromolecules 1995, 28, 1066-1075.

${ }^{92}$ Semenov, A. N.; Rubinstein, M. Dynamics of Entangled Associating Polymers with Large Aggregates. Macromolecules 2002, 35, 4821-4837.

${ }^{93}$ Tanaka, F. Polymer Physics, 1st ed.; Cambridge University Press: Cambridge, U.K., 2011. 
${ }^{94}$ Macosko, C.W. Rheology: Principles, Measurements, and Applications, 1st ed.; Wiley-VCH: New York, NY, 1994.

${ }^{95}$ Münstedt, H. Rheological Experiments at Constant Stress as Efficient Method to Characterize Polymeric Materials. J. Rheol. 2014, 58, 565-587.

${ }^{96}$ Mavridis, H.; Shroff, R. N. Temperature Dependence of Polyolefin Melt Rheology. Polym. Eng. Sci. 1992, 32, 1778-1791.

${ }^{97}$ van Gurp, M.; Palmen, J. Time-temperature superposition for polymeric blends. Rheol. Bull. 1998, 67, 5-8

${ }^{98}$ Hiemenz, P. C.; Lodge, T. P. Polymer Chemistry, 2nd ed.; CRC Press, Taylor \& Francis Group: Boca Raton, FL, 2007.

${ }^{99}$ Baravian, C.; Benbelkacem, G.; Caton, F. Unsteady Rheometry: Can We Characterize Weak Gels with a Controlled Stress Rheometer? Rheol. Acta 2007, 46, 577-581.

${ }^{100}$ Semenov, A. N.; Rubinstein, M. Dynamics of Entangled Associating Polymers with Large Aggregates. Macromolecules 2002, 35, 4821-4837.

${ }^{101}$ Amin, D.; Likhtman, A. E.; Wang, Z. Dynamics in Supramolecular Polymer Networks Formed by Associating Telechelic Chains. Macromolecules 2016, 49, 7510-7524.

${ }^{102}$ Milner, S. T.; Xi, H. How Copolymers Promote Mixing of Immiscible Homopolymers. $J$. Rheol. 1996, 40, 663-687.

${ }^{103}$ Milner, S. T. How Do Copolymer Compatibilizers Really Work? MRS Bull. 1997, 22, 38-42.

${ }^{104}$ Bates, F. S. (Workshop Chair). Frontiers in Polymer Science and Engineering, Report of a 2016 NSF Workshop (Frontiers in Polymer Science and Engineering, University of Minnesota-Twin Cities, Minneapolis, 2017). 
TOC Graphic

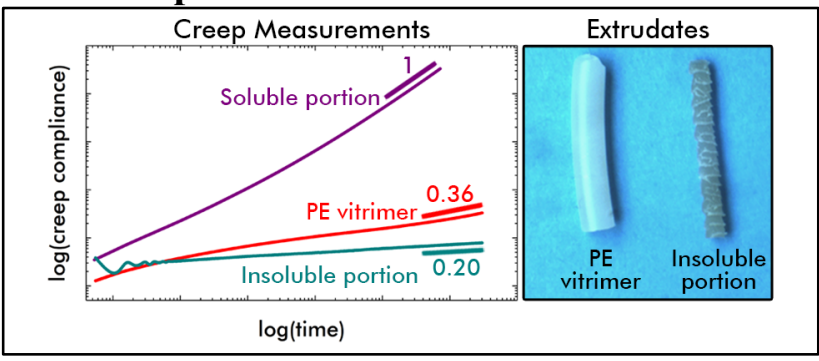


Supplementary information for

\title{
Linear viscoelasticity and flow of self-assembled vitrimers: the case of a polyethylene/dioxaborolane system
}

\author{
Ralm G. Ricarte, ${ }^{\dagger}$ François Tournilhac, ${ }^{\dagger}$ Michel Cloître, ${ }^{\dagger}$ and Ludwik Leibler ${ }^{\ddagger}$ \\ ${ }^{\dagger}$ Molecular, Macromolecular Chemistry, and Materials Laboratory, CNRS, ESPCI-Paris, PSL \\ Research University, 10 Rue Vauquelin, 75005 Paris, France and *UMR CNRS 7083 Gulliver, \\ ESPCI Paris, PSL Research University, 10 Rue Vauquelin, 75005 Paris, France
}

Email: ralm.ricarte@espci.fr and ludwik.leibler@espci.fr 


\section{Table of contents}

Fourier transform infrared spectra of PE, PE $+0.05 \mathrm{wt} \%$ DCP, PE-g, and blends $\quad$ S3

$\begin{array}{ll}\text { Strain sweeps } & \text { S3 }\end{array}$

$\begin{array}{ll}\text { Supplementary rheology data } & \text { S5 }\end{array}$

$\begin{array}{ll}\text { Polyethylene } & \text { S5 }\end{array}$

$\begin{array}{ll}\mathrm{PE}+0.05 \mathrm{wt} \% \mathrm{DCP} & \mathrm{S} 10\end{array}$

$\begin{array}{ll}\text { PE-g } g & \text { S14 }\end{array}$

$\begin{array}{ll}\text { PE- } v & \text { S17 }\end{array}$

$\begin{array}{lr}\text { PE-v-sol } & \text { S19 }\end{array}$

$\begin{array}{ll}\text { PE-v-insol } & \text { S23 }\end{array}$

$\begin{array}{lr}\text { Blend PE/PE-v-insol } & \text { S25 }\end{array}$

$\begin{array}{lr}\text { Blend PE-v-sol/PE- } v \text {-insol } & \text { S27 }\end{array}$

Influence of thermal degradation on PE-g and blend PE-v-sol/PE-v-insol S29

Comparison of PE, PE-g, PE- $v$, PE- $v$-sol, and PE- $v$-insol rheology $\quad$ S31

Comparison of PE- $v$ and blend PE- $v$-sol/PE- $v$-insol rheology $\quad$ S33

$\begin{array}{ll}\text { Conversion of creep compliance to dynamic moduli } & \text { S34 }\end{array}$

$\begin{array}{ll}\text { Estimation of cross-link densities } & \text { S35 }\end{array}$

$\begin{array}{ll}\text { References } & \text { S36 }\end{array}$ 


\section{Fourier transform infrared spectra of PE, PE $+0.05 \mathrm{wt} \% \mathrm{DCP}, \mathrm{PE}-\mathrm{g}$, and blends}

Figure S1A features the FTIR spectra for polyethylene (PE), PE $+0.05 \mathrm{wt} \%$ dicumyl peroxide (DCP), and graft functionalized PE (PE-g), while Figure S1B displays the spectra for blends of PE/PE-v-insol and PE-v-sol/PE-v-insol.
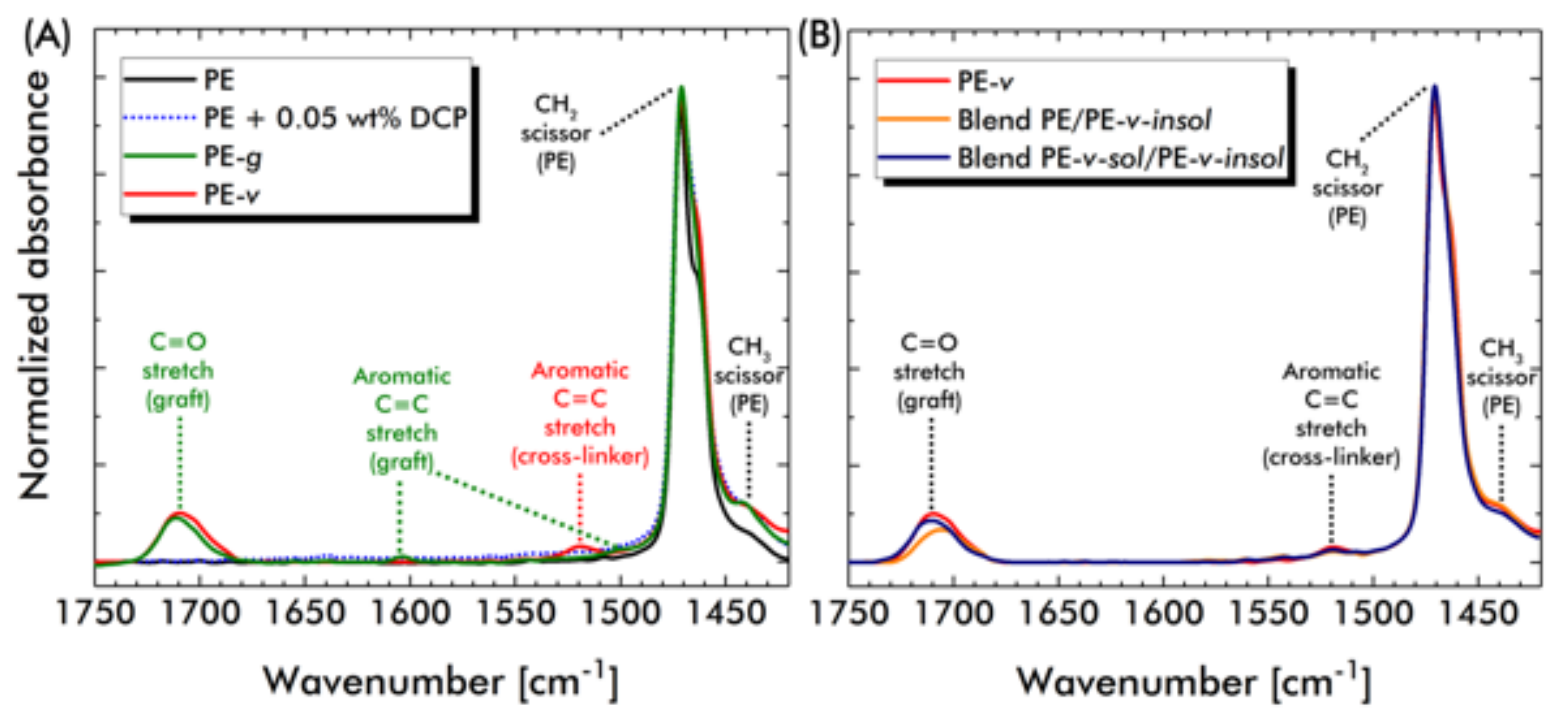

Figure S1. FTIR spectra of (A) polyethylene (PE), PE + $0.05 \mathrm{wt} \%$ dicumyl peroxide (DCP), PE-g, (B) blend PE/PE- $v$-insol, and blend PE- $v$-sol/PE- $v$-insol. In each panel, the FTIR spectrum of PE- $v$ from Figure 3 is reproduced for comparison. Characteristic signals of the PE backbone, graft, and cross-linker components are highlighted.

\section{Strain sweeps}

Figure S2 contains strain sweep complex modulus $\left(G^{*}\right)$ data for PE, PE $+0.05 \mathrm{wt} \%$ DCP, PE- $g$, and PE-v. Figure S3 has the strain sweep data for PE- $v$-sol, PE- $v$-insol, blend PE/PE- $v$-insol, and blend PE-v-sol/PE-v-insol. 

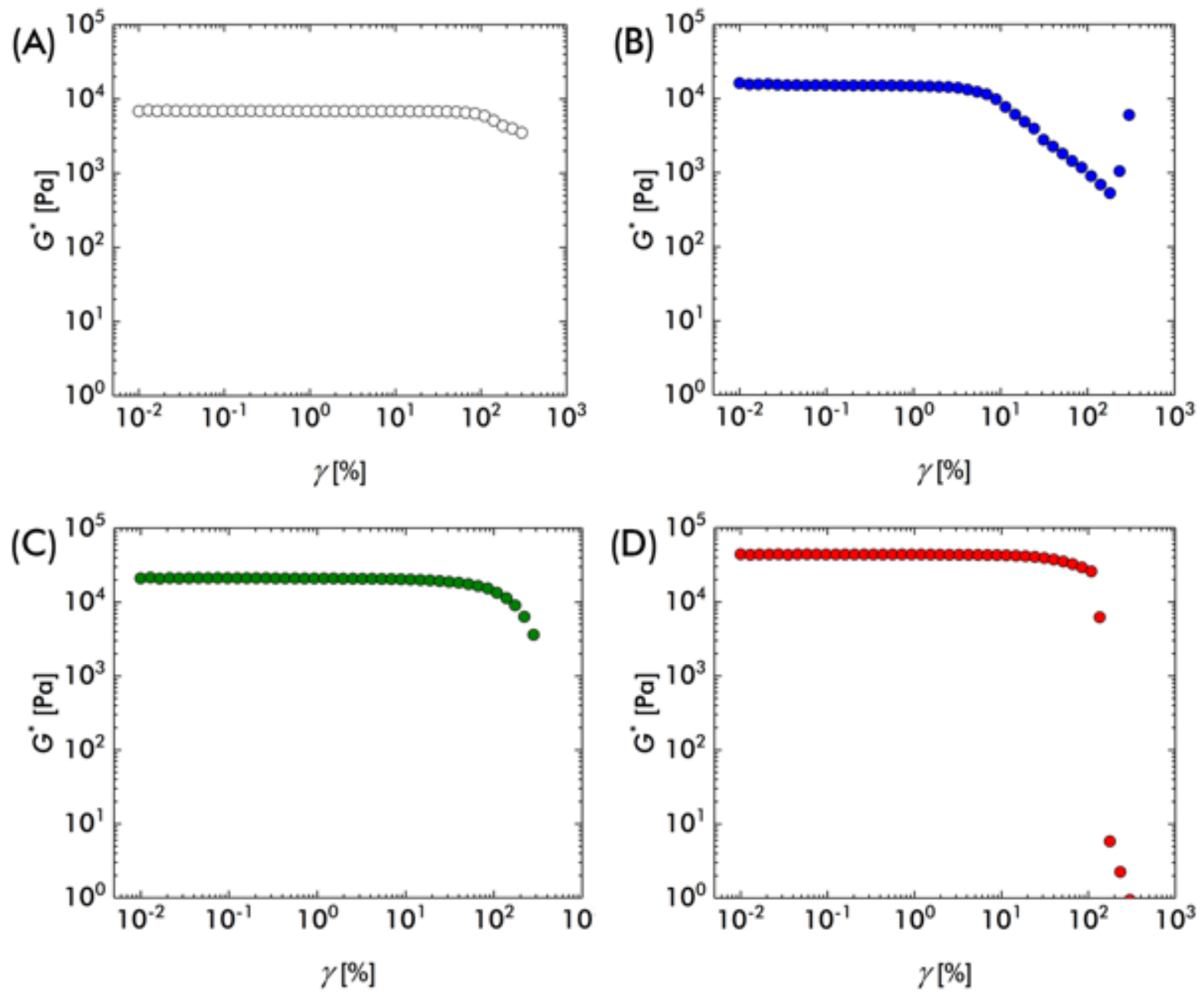

Figure S2. Strain sweep $G^{*}$ data for (A) PE, (B) PE $+0.05 \mathrm{wt} \%$ DCP, (C) PE-g, and (D) PE-v. All measurements were performed at $T=140{ }^{\circ} \mathrm{C}$ and $\omega=1 \mathrm{rad} / \mathrm{s}$. 

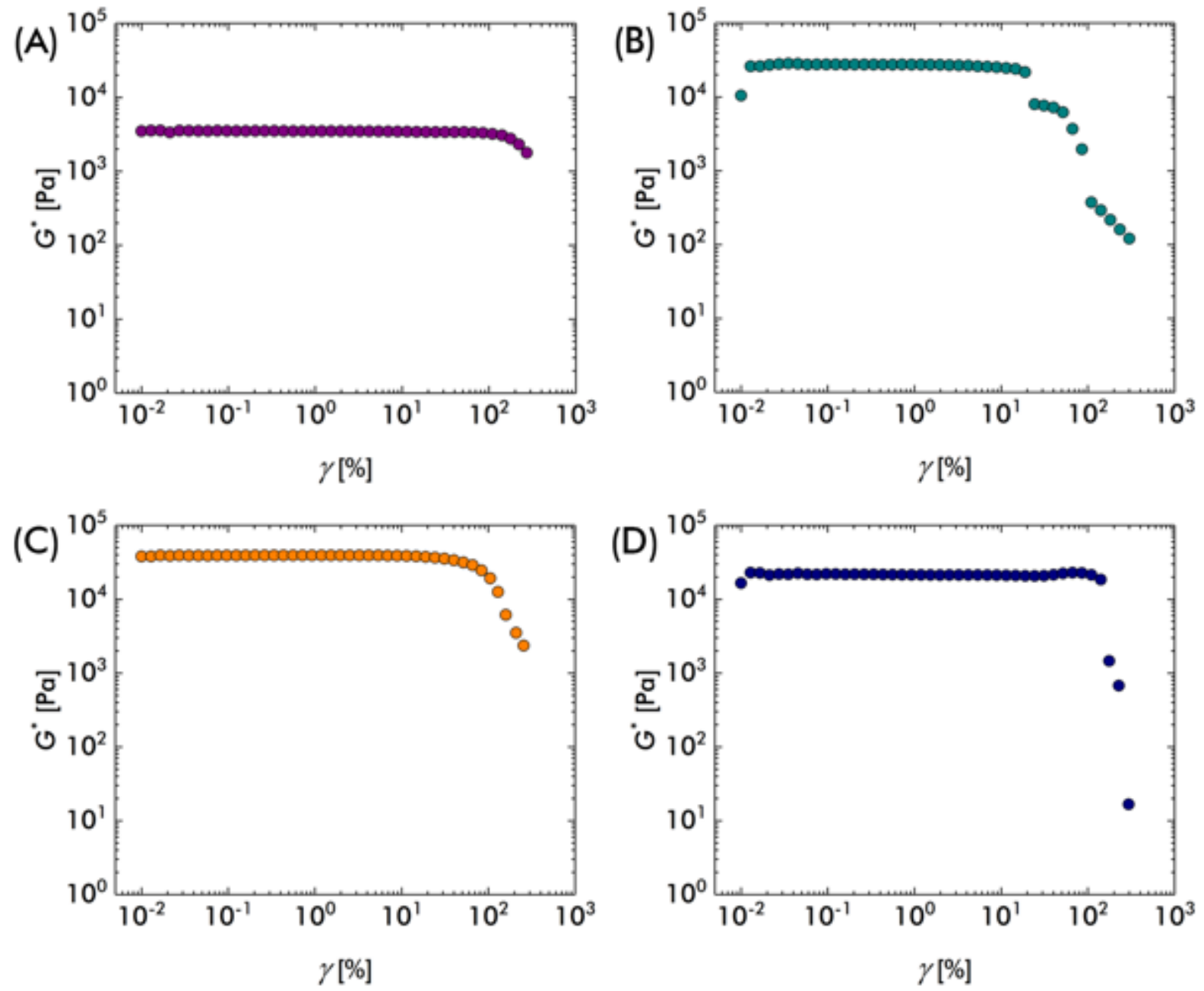

Figure S3. Strain sweep $G^{*}$ data for (A) PE-v-sol, (B) PE-v-insol, (C) blend PE/PE-v-insol, and (D) blend PE- $v$-sol/PE- $v$-insol. All measurements were performed at $T=140{ }^{\circ} \mathrm{C}$ and $\omega=1$ $\mathrm{rad} / \mathrm{s}$.

\section{Supplementary rheology data}

Polyethylene. Small-amplitude oscillatory shear (SAOS) data for PE are displayed in Figure S4. The unshifted dynamic moduli and $\tan \delta$ are seen in Figure S4A and S4B, respectively. The shifted $\tan \delta$ in Figure S4C were calculated using the horizontal shift factors $\left(a_{T}\right)$ seen in Figure 4A in the main text. Additionally, the phase angle $(\delta)$ versus the magnitude of $G^{*}$ are plotted in Figure S4D. The $\mathrm{x}$ - and y-axes in this format (known as a van Gurp-Palmen plot) represent the variables 
that are directly measured during SAOS. In a van Gurp-Palmen plot, collapse of the data into a single curve suggests time-temperature superposition (tTs) applies, while the lack of overlap implies the failure of tTs. ${ }^{\mathrm{S} 1}$ The collapse of the data points in both Figures S4C and S4D indicates that PE obeys tTs.
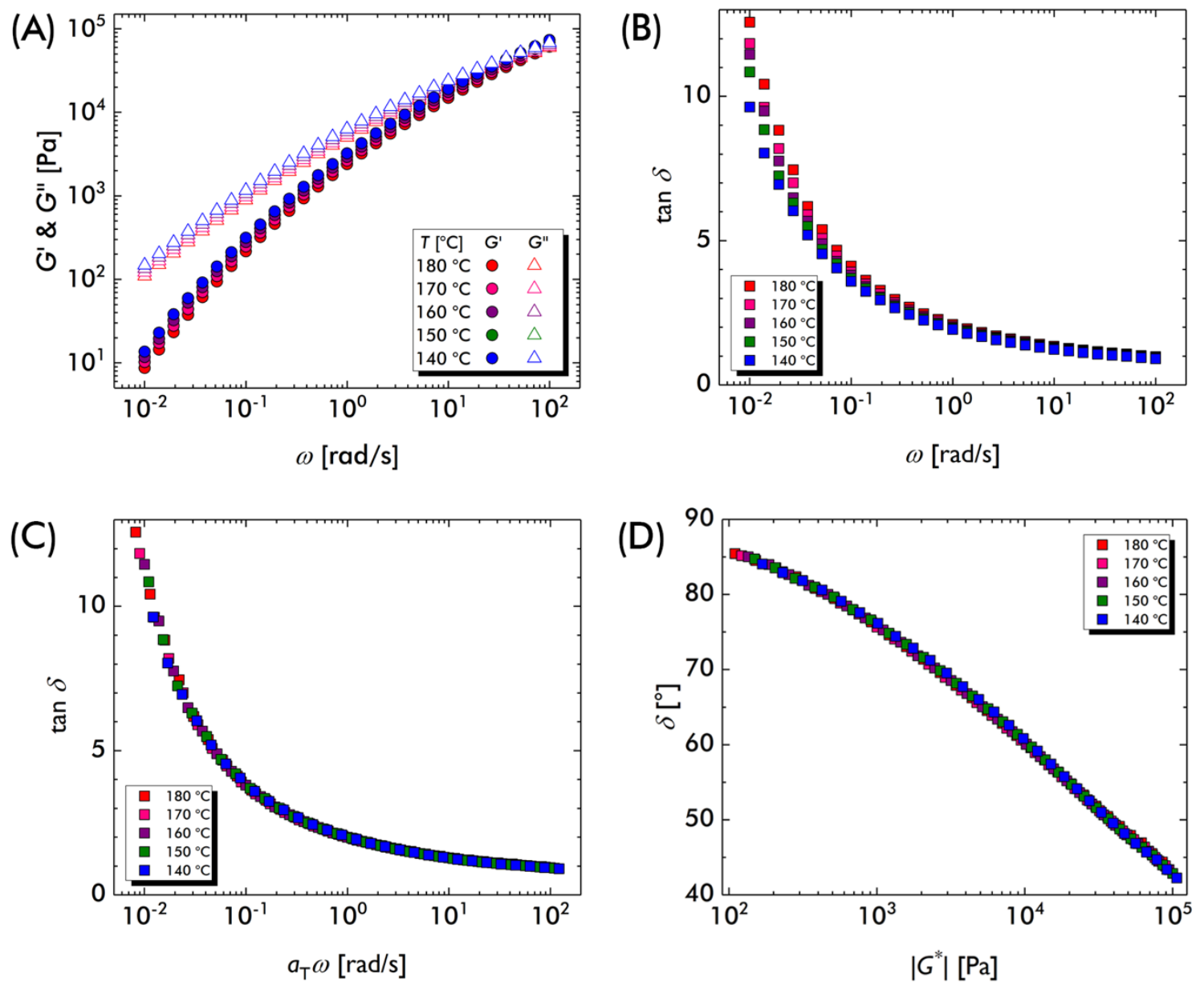

Figure S4. SAOS data for PE. (A) Elastic $\left(G^{\prime}\right)$ and viscous $\left(G^{\prime \prime}\right)$ moduli, (B) unshifted and (C) shifted $\tan \delta$, and (D) the van Gurp-Palmen plot. In (C) and (D), complete overlap of data suggests that PE obeys time-temperature superposition.

Unshifted stress relaxation curves for PE are shown in Figure S5. For creep and recovery measurements, the raw strain data are displayed in Figure S6A. For data measured at $180{ }^{\circ} \mathrm{C}$, the 
creep recovery compliance $\left(J_{r}(t)\right)$, true creep recovery compliance $\left(J_{r}^{\text {true }}(t)\right)$, and linear slope of the creep recovery compliance $\left(\frac{d J_{r}}{d t_{r}}\right)$ are plotted in Figure S6B. Unshifted $J(t)$ are shown in Figure S7. Figures S8A and S8B show the linear regressions used to calculate the steady-state creep compliance $\left(J_{e}^{0}\right)$ and zero-shear viscosity $\left(\eta_{0}\right)$, respectively. Figure S9 discloses the estimated values of $J_{e}^{0}, \eta_{0}$, and terminal relaxation time $(\tau)$, along with the linear regression used to estimate the flow activation energy $\left(\Delta E_{a c t}\right)$.

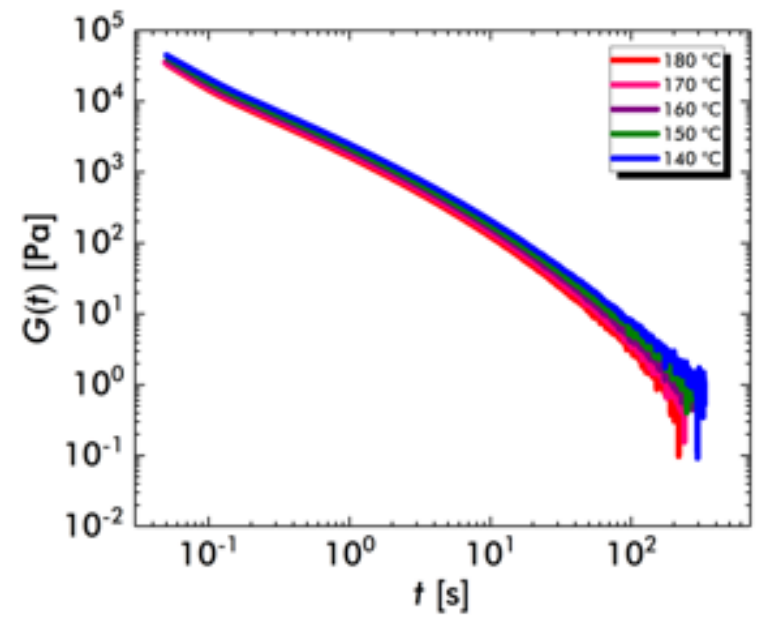

Figure S5. Stress relaxation of PE. 

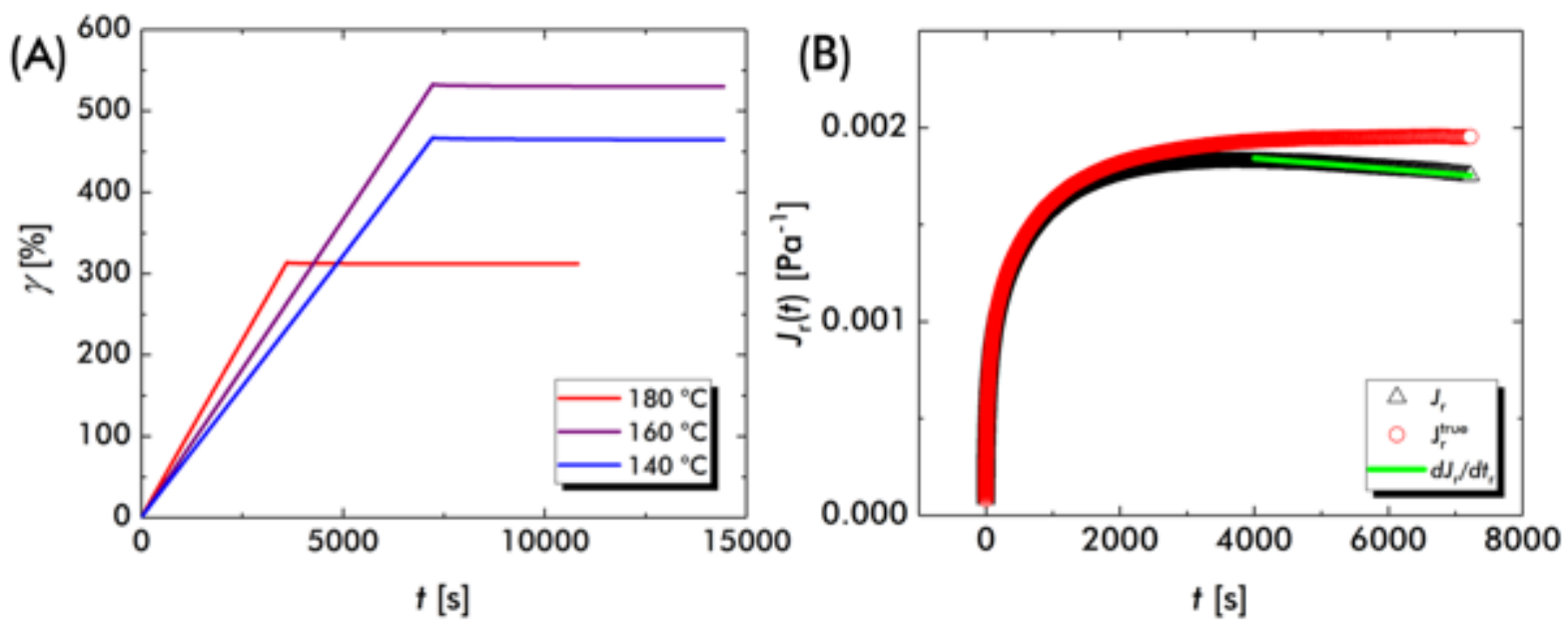

Figure S6. (A) Raw strain measurements during creep and recovery of PE. $\sigma=10 \mathrm{~Pa}$ for all temperatures. (B) Calculation of $J_{r}^{\text {true }}$ for PE at $T=180^{\circ} \mathrm{C}$.

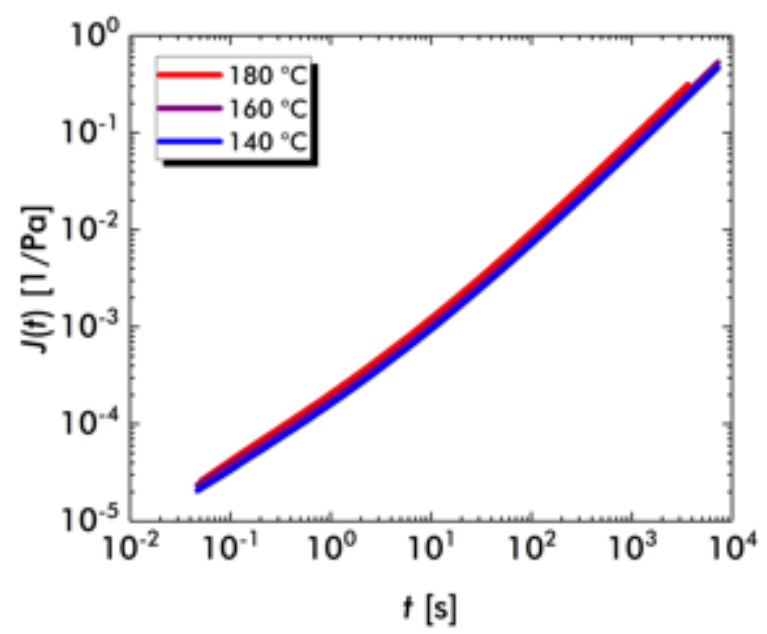

Figure S7. Unshifted creep compliance data for PE. $\sigma=10 \mathrm{~Pa}$ for all temperatures. 

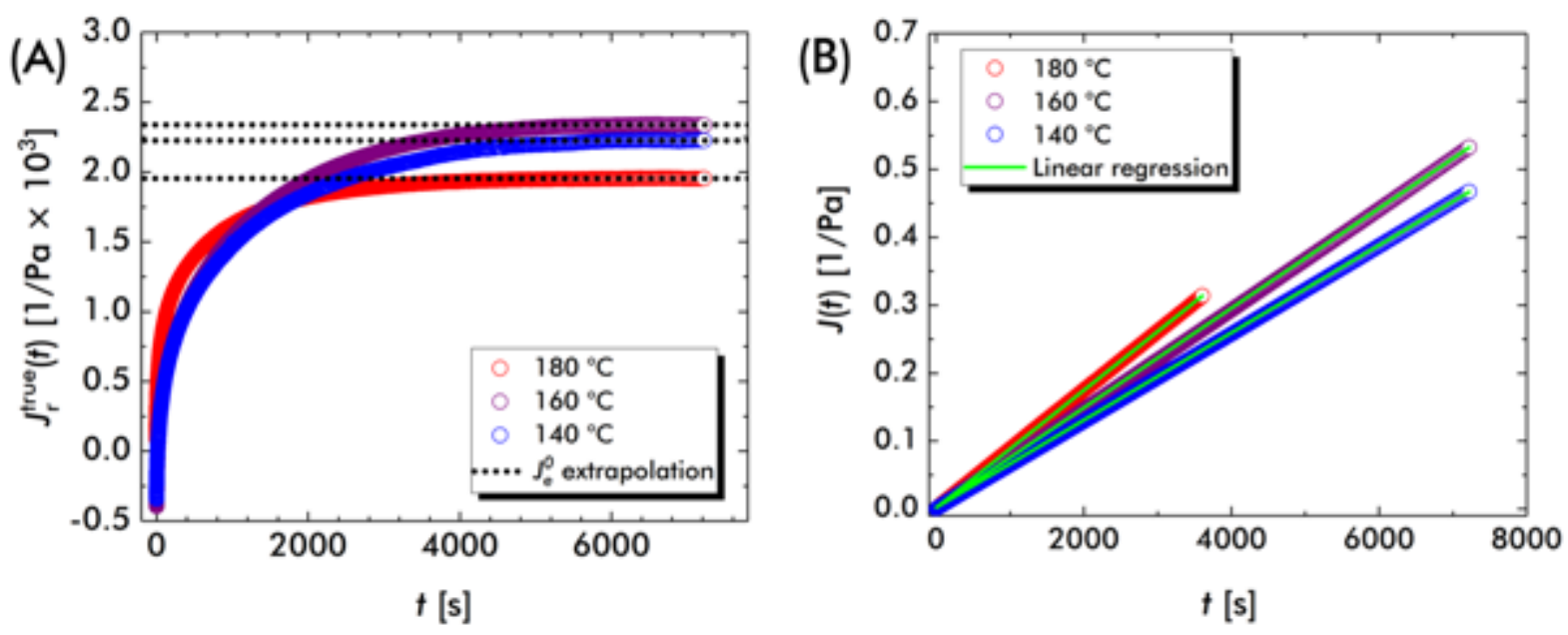

Figure S8. Creep compliance and recovery data for PE at varying temperature. (A) Creep recovery. Dotted lines represent the plateau extrapolation used to calculate $J_{e}^{0}$. (B) Creep compliance. Green lines signify the linear extrapolations used to estimate $\eta_{0}$. 

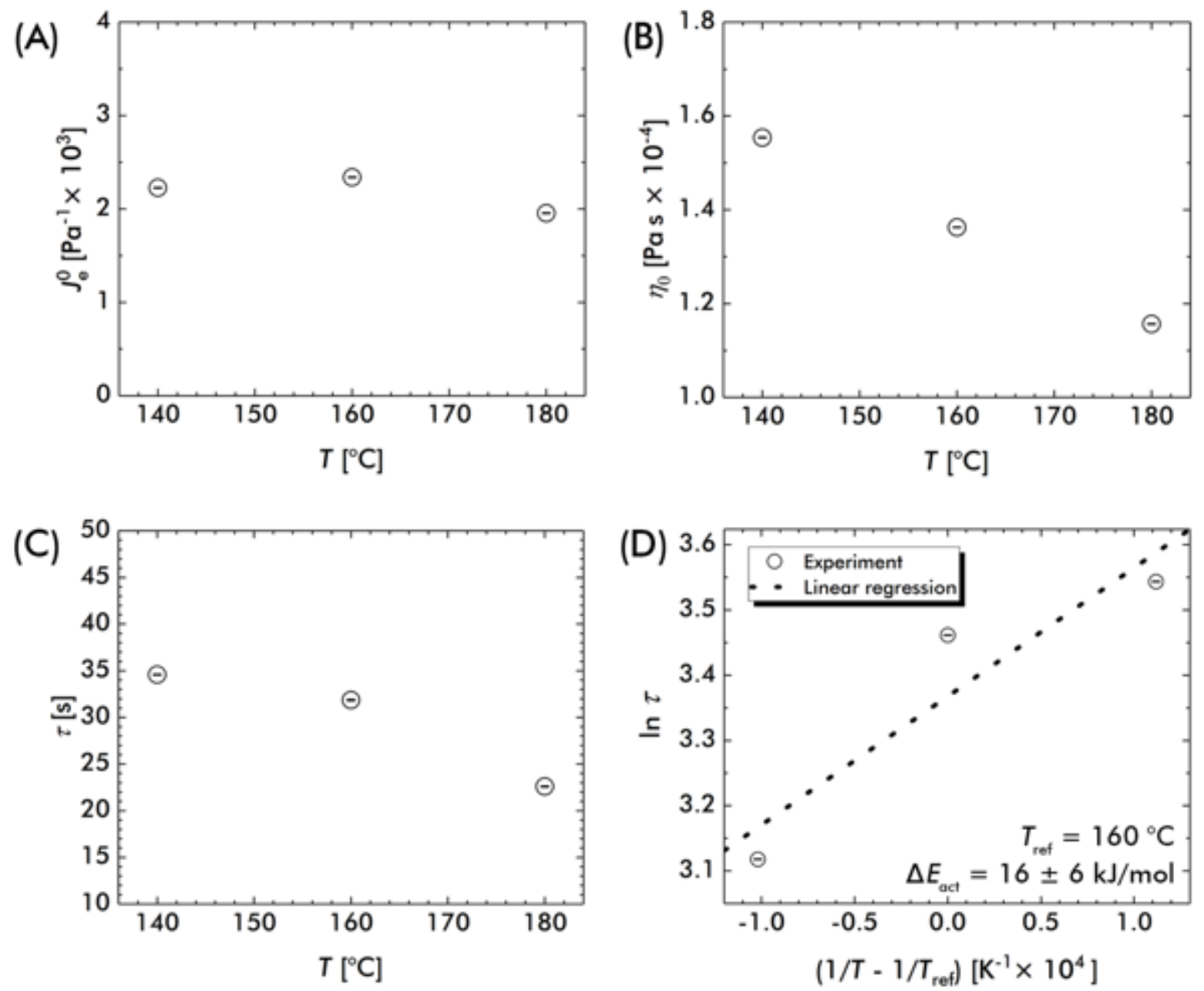

Figure S9. PE macroscopic flow properties as determined by creep compliance and recovery measurements. (A) Steady-state linear recoverable compliance $\left(J_{e}^{0}\right),(\mathrm{B})$ zero-shear viscosity $\left(\eta_{0}\right)$, and $(\mathrm{C})$ terminal relaxation time $(\tau)$. (D) Fitting of $\tau$ to Eqn. 10. A flow activation energy $\left(\Delta E_{a c t}\right)$ of $16 \pm 6 \mathrm{~kJ} / \mathrm{mol}$ was determined.

$P E+0.05 w t \%$ DCP. Unshifted dynamic moduli and $\tan \delta$ for PE $+0.05 \mathrm{wt} \%$ DCP are displayed in Figures S10A and S10B. When the PE $a_{T}$ are applied to the PE $+0.05 \mathrm{wt} \%$ DCP data (Figure S10C), overlap does not occur. The data in the van Gurp-Palmen plot also do not superimpose, suggesting the sample does not obey tTs. The failure of tTs is typically seen when DCP is added to neat PE. ${ }^{\mathrm{S} 1}$ Consistent with previous work by van Gurp and Palmen, we hypothesize that PE + 
$0.05 \mathrm{wt} \%$ DCP contains a dilute concentration of branched chains, which have a different $\Delta E_{\text {act }}$ than the linear chains. ${ }^{\mathrm{S} 1}$
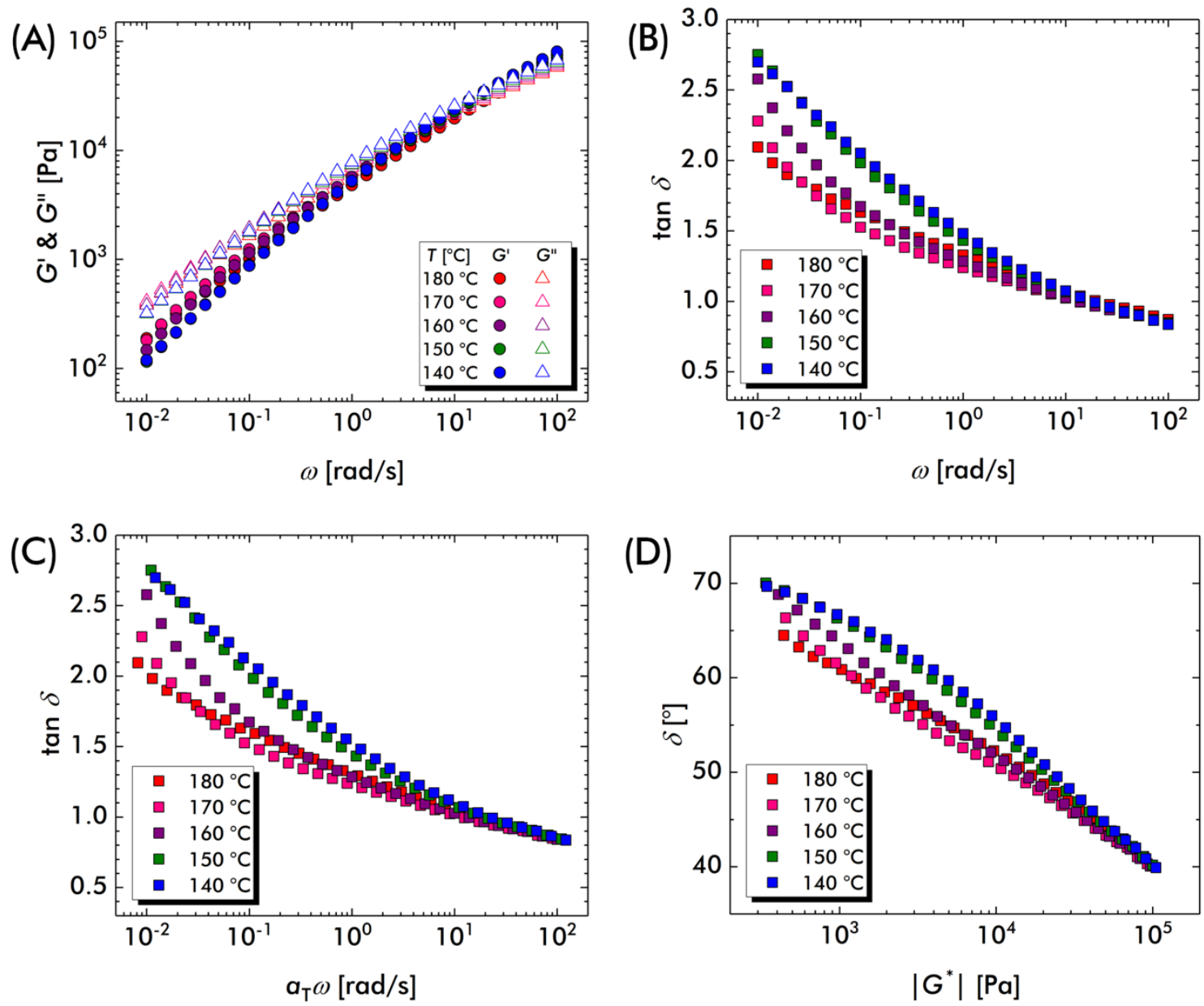

Figure S10. SAOS data for PE $+0.05 \mathrm{wt} \% \mathrm{DCP}$. (A) $G^{\prime}$ and $G^{\prime \prime}$ and (B) unshifted $\tan \delta$, (C) shifted $\tan \delta$ using the $a_{T}$ calculated for neat PE, and (D) the van Gurp-Palmen plot.

Unshifted stress relaxation data for PE $+0.05 \mathrm{wt} \%$ DCP are displayed in Figure S11. For creep and recovery measurements, the raw strain and $J(t)$ are plotted in Figures S12A and S12B, respectively. Linear regressions used to estimate $J_{e}^{0}$ and $\eta_{0}$ are shown in Figures S13A and S13B. 
$J_{e}^{0}, \eta_{0}, \tau$, and the linear regression used to estimate $\Delta E_{a c t}$ are featured in Figure S14. $\Delta E_{\text {act }}$ was estimated to be $16 \pm 1 \mathrm{~kJ} / \mathrm{mol}$, which matches the value for neat PE.

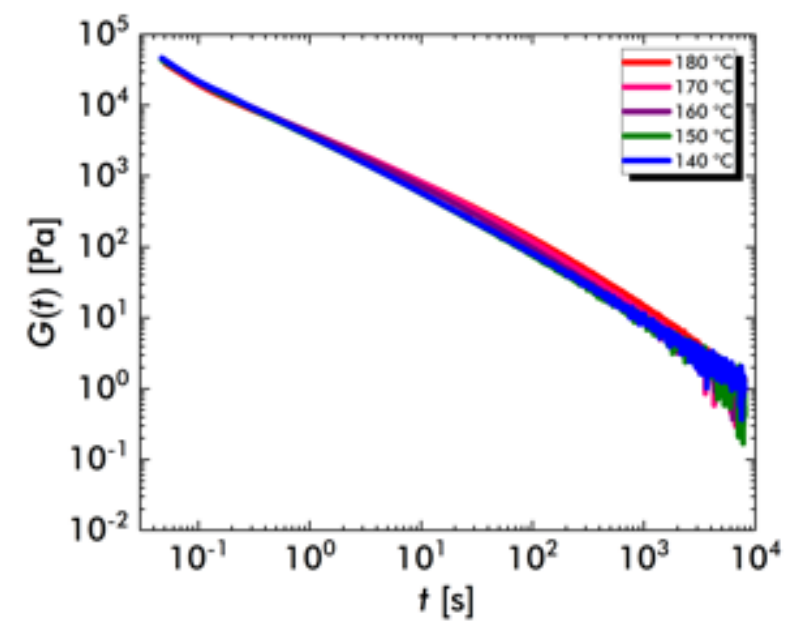

Figure S11. Stress relaxation of PE $+0.05 \mathrm{wt} \%$ DCP.
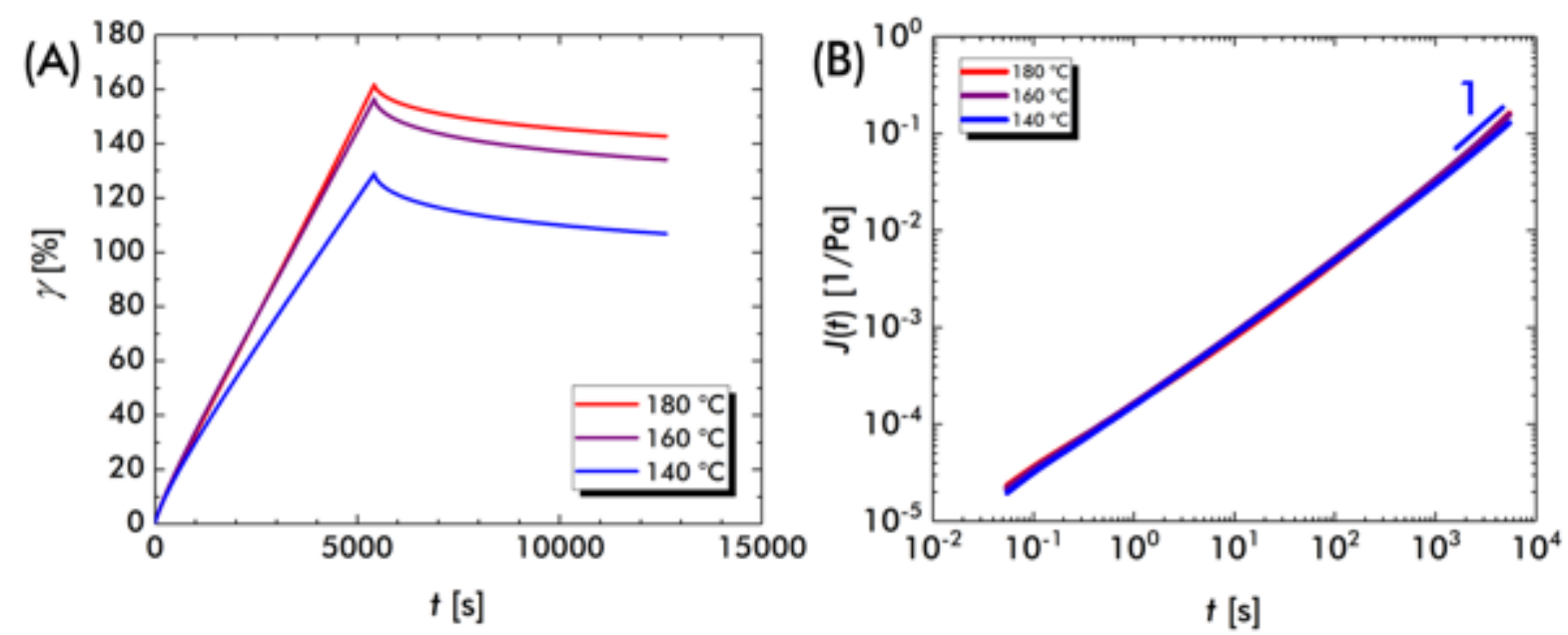

Figure S12. (A) Raw strain measurements during creep and recovery of PE $+0.05 \mathrm{wt} \% \mathrm{DCP}$. $\sigma=10 \mathrm{~Pa}$ for all temperatures. (B) Creep compliance data for PE $+0.05 \mathrm{wt} \% \mathrm{DCP}$. 

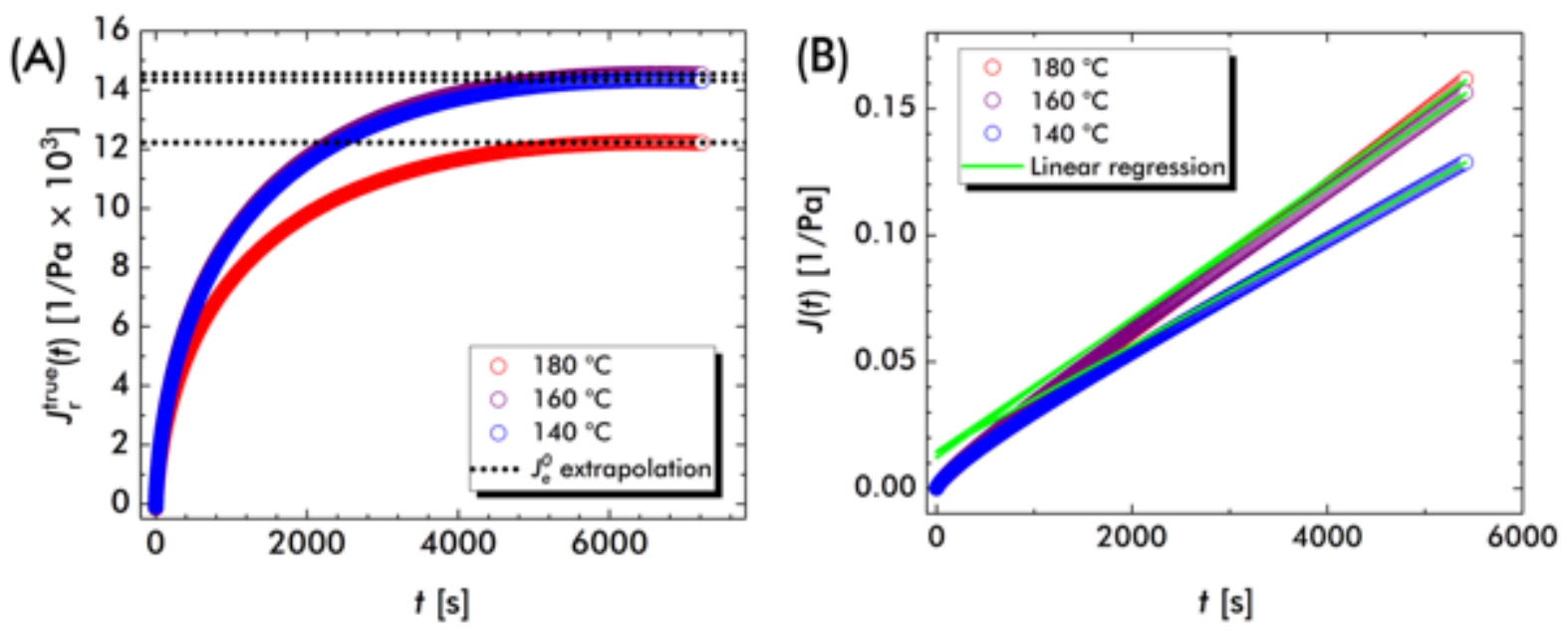

Figure S13. Creep compliance and recovery data for PE $+0.05 \mathrm{wt} \%$ DCP at varying temperature. (A) Creep recovery. Dotted lines represent the plateau extrapolation used to calculate $J_{e}^{0}$. (B) Creep compliance. Green lines signify the linear extrapolations used to estimate $\eta_{0}$ 

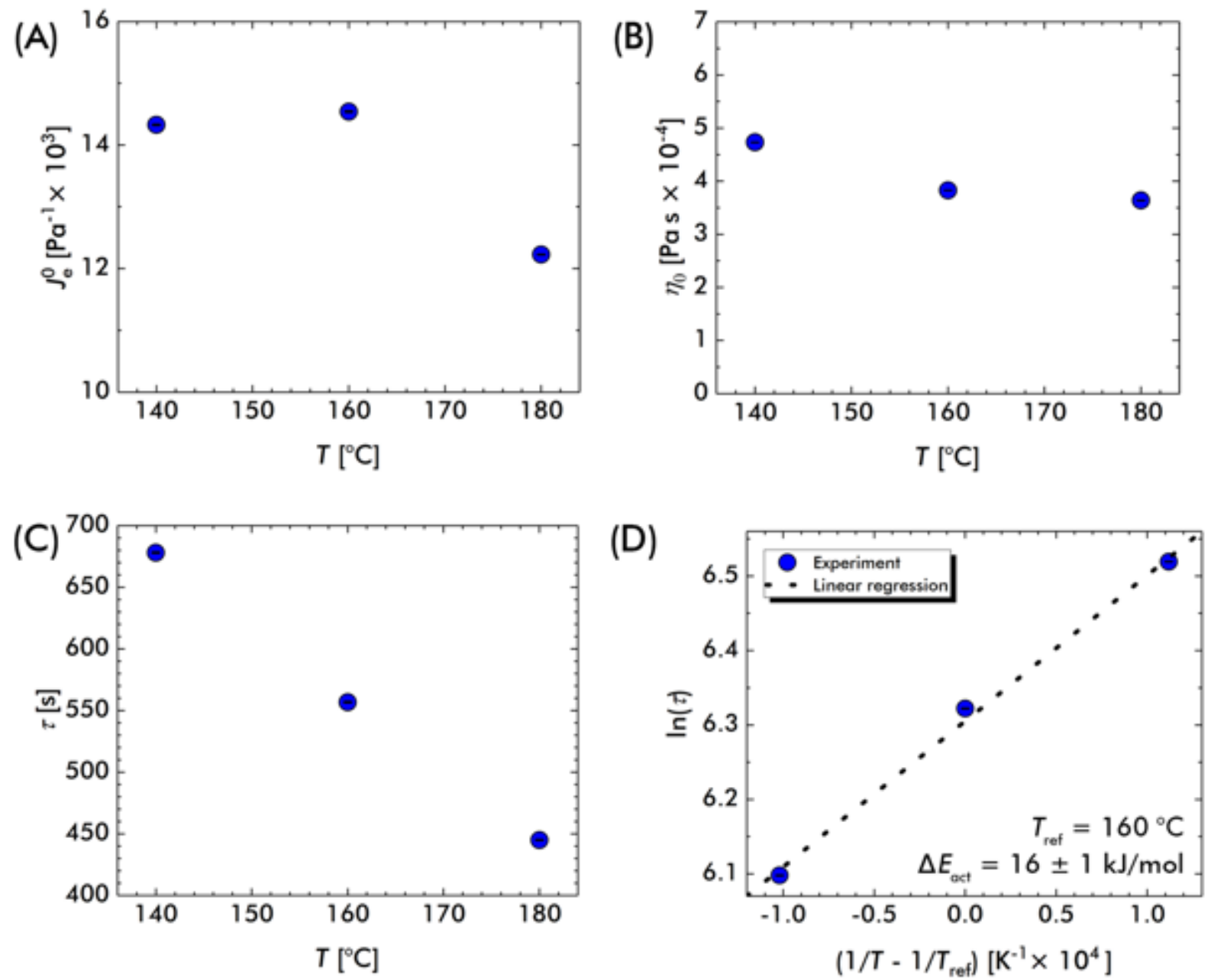

Figure S14. PE $+0.05 \mathrm{wt} \%$ DCP macroscopic flow properties as determined by creep compliance and recovery measurements. (A) $J_{e}^{0}$, (B) $\eta_{0}$, and (C) $\tau$. (D) Fitting of $\tau$ to Eqn. 10. A flow activation energy $\left(\Delta E_{a c t}\right)$ of $16 \pm 1 \mathrm{~kJ} / \mathrm{mol}$ was determined.

$P E$-g. Unshifted $\tan \delta$ data are shown in Figure S15A. The lack of data overlap in the van GurpPalmen plot (Figure S15B) indicates that tTs fails for PE-g. Figure S16 displays the raw strain values measured during creep and recovery measurements. Figure $\mathrm{S} 17$ shows $J(t)$ at $140{ }^{\circ} \mathrm{C}$ for a period of $20 \mathrm{~h}$. Although the measurement is exceptionally long, PE-g still did not achieve the steady-state flow scaling of 1 . For $T \geq 160{ }^{\circ} \mathrm{C}$, creep and recovery measurements were used to 
estimate macroscopic flow properties. Figures S18A and S18B display the linear regressions used to estimate $J_{e}^{0}$ and $\eta_{0}$. Figure $\mathrm{S} 19$ features $J_{e}^{0}, \eta_{0}$, and $\tau$.
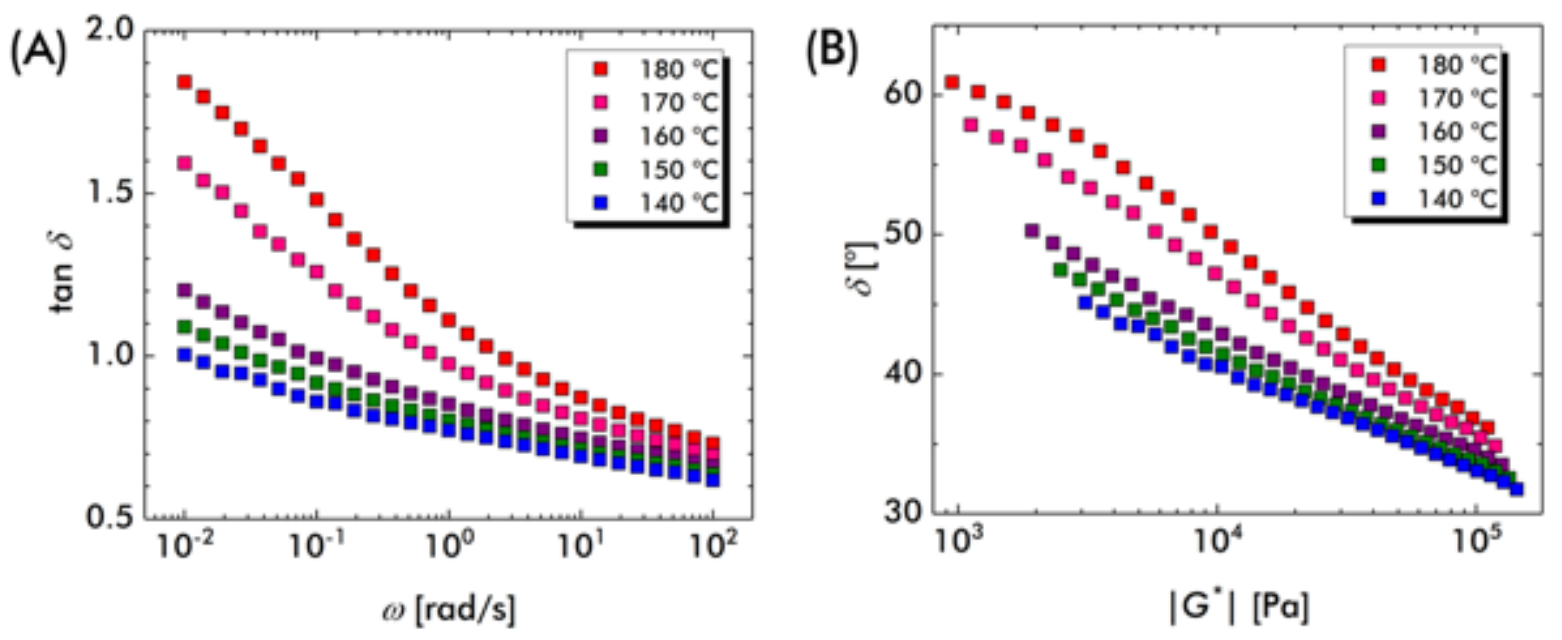

Figure S15. SAOS data for PE-g. (A) Unshifted $\tan \delta$ and (B) the van Gurp-Palmen plot.

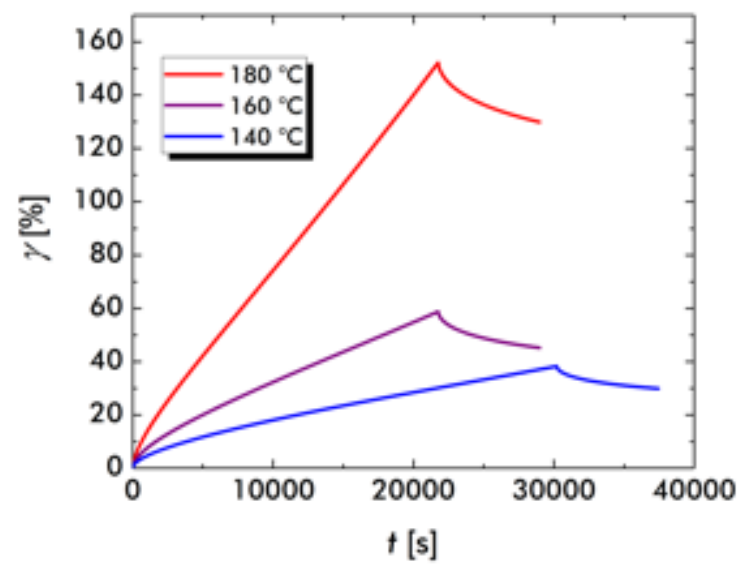

Figure S16. Raw strain measurements during creep and recovery of PE- $g . \sigma=30 \mathrm{~Pa}$ for all temperatures. 


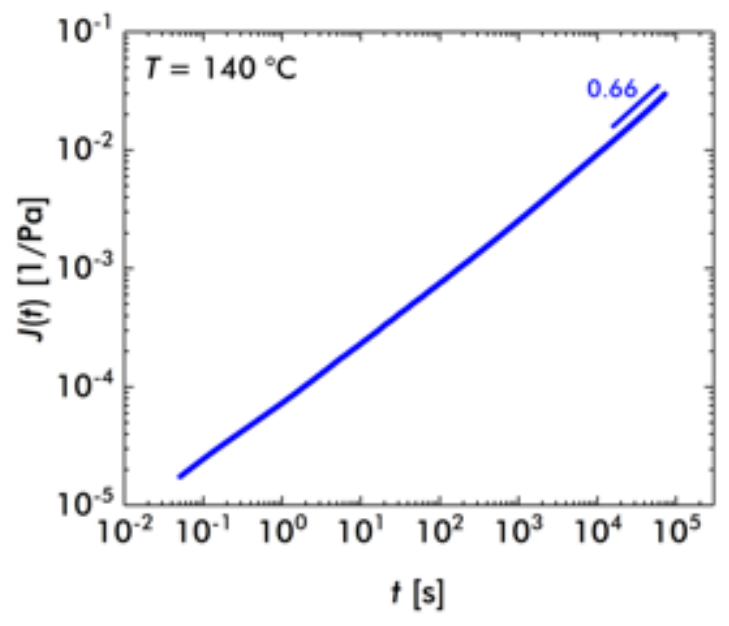

Figure S17. $20 \mathrm{~h}$ creep compliance of PE- $g$ at $T=140{ }^{\circ} \mathrm{C}$ and $\sigma=30 \mathrm{~Pa}$.
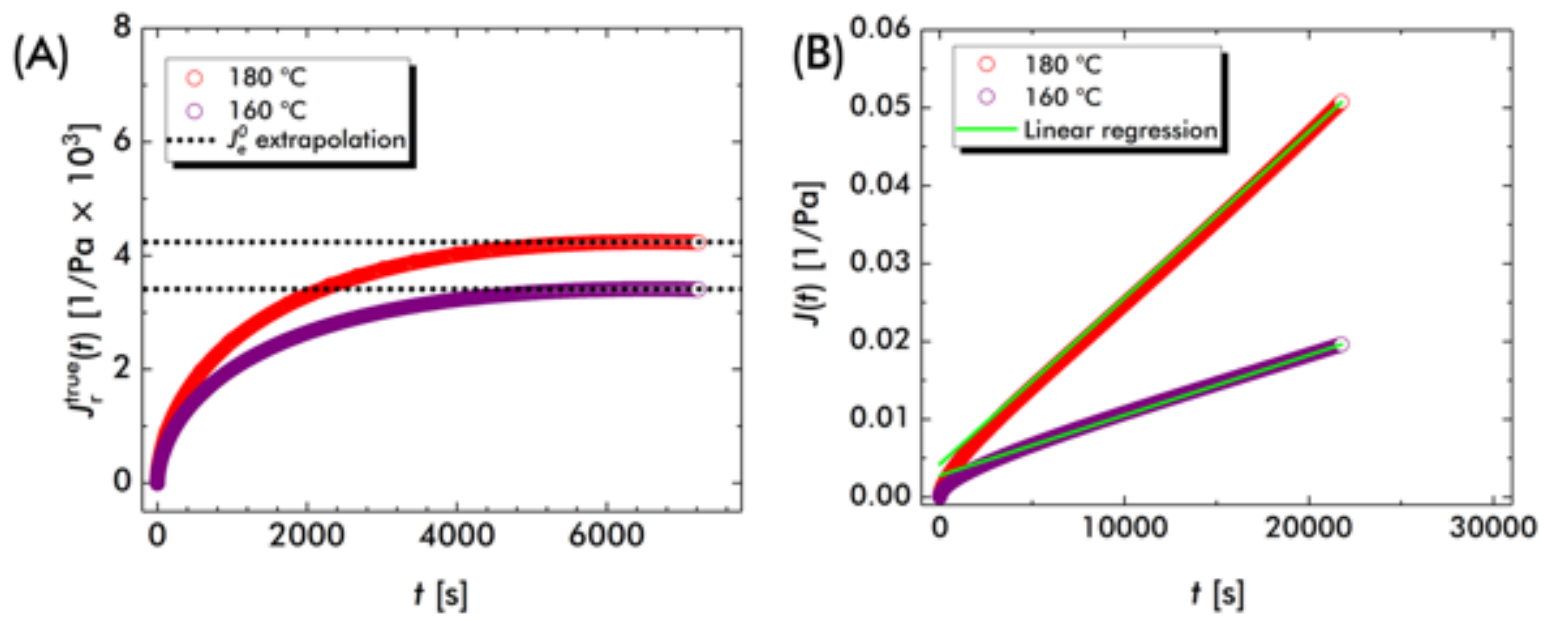

Figure S18. Creep compliance and recovery data for PE-g at varying temperature. (A) Creep recovery. Dotted lines represent the plateau extrapolation used to calculate $J_{e}^{0}$. (B) Creep compliance. Green lines signify the linear extrapolations used to estimate $\eta_{0}$. 

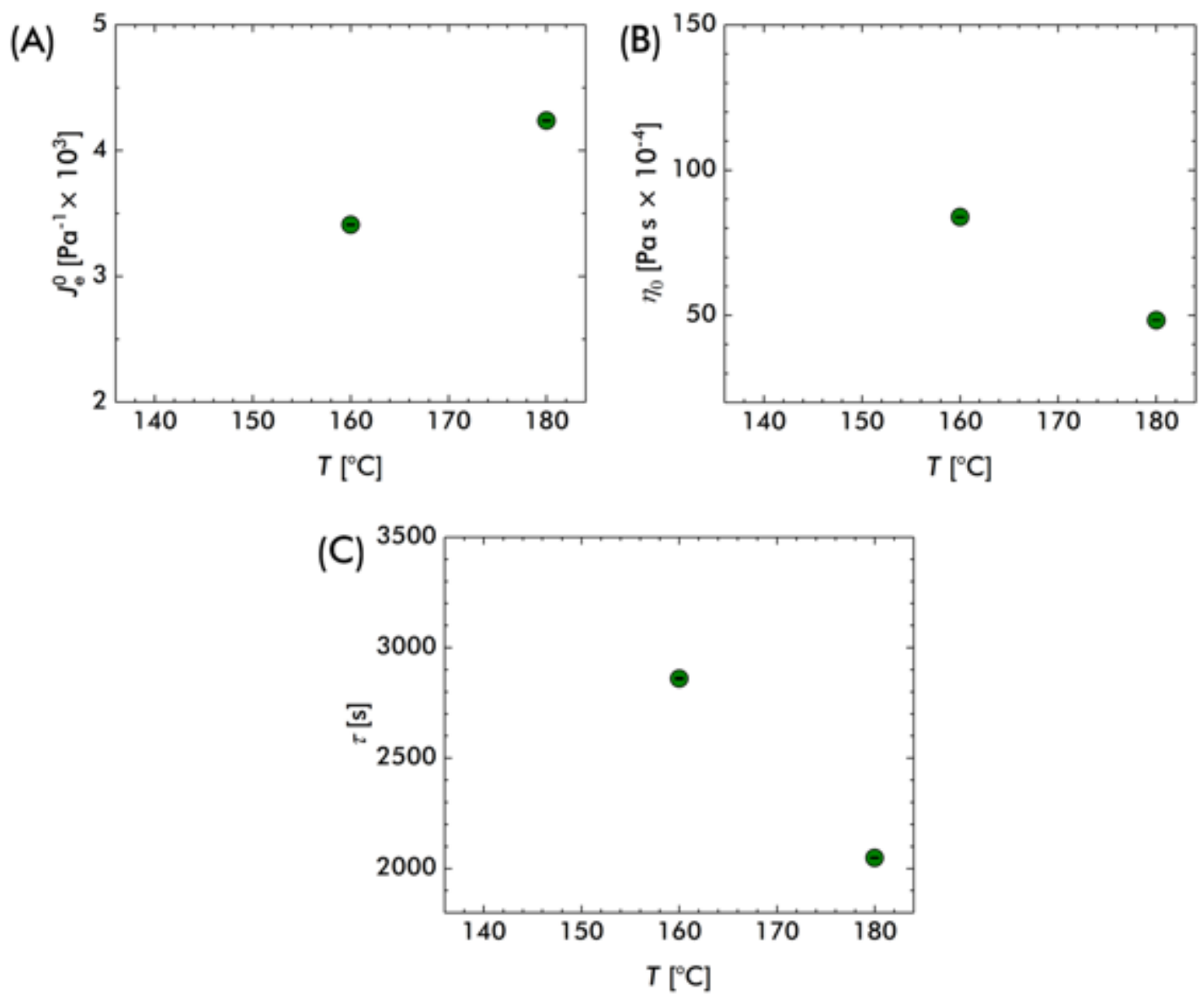

Figure S19. PE-g macroscopic flow properties as determined by creep compliance and recovery measurements. (A) $J_{e}^{0}$, (B) $\eta_{0}$, and (C) $\tau$.

$P E-v$. Unshifted $\tan \delta$ data are featured in Figure S20A. The lack of data overlap in the van GurpPalmen plot (Figure S20B) indicates that PE- $v$ does not obey tTs. Figure S21 features PE- $v$ stress relaxation measurements performed for a duration of $8 \mathrm{~h}$. Figure S22 shows the raw strain values measured during creep and recovery measurements. 

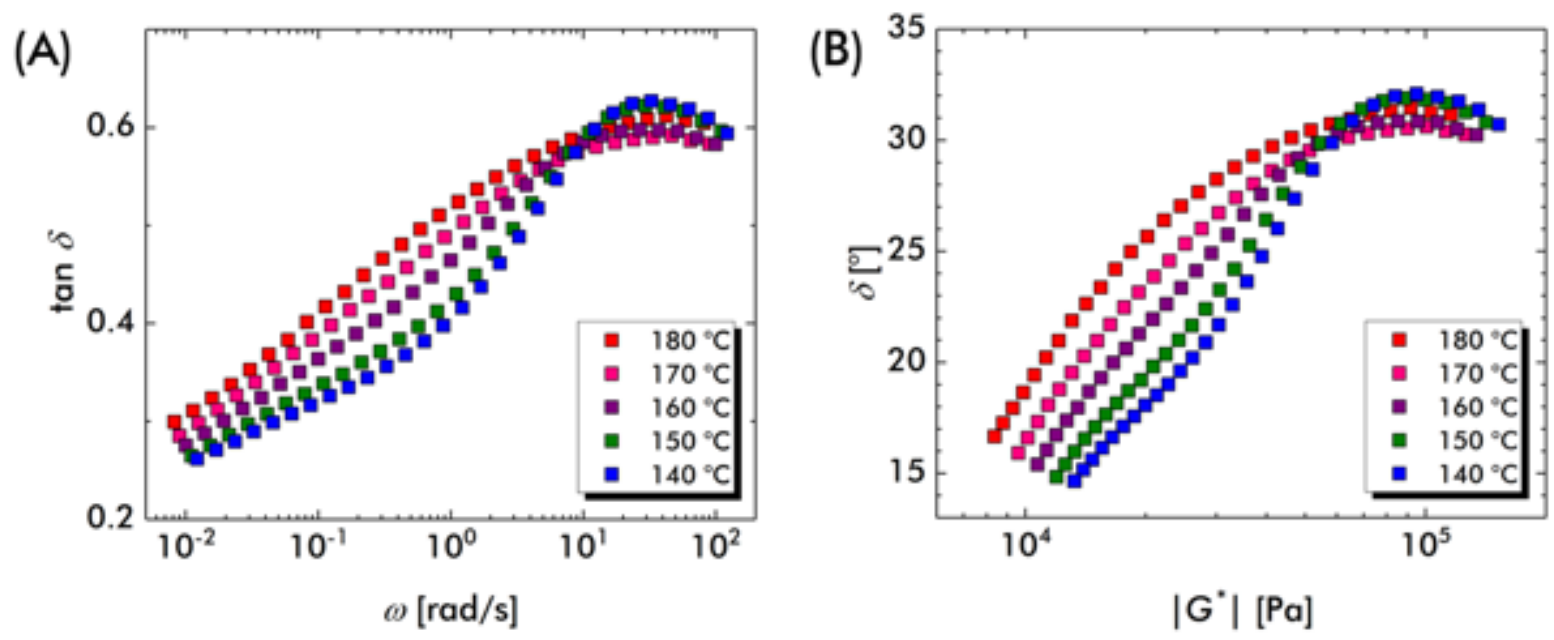

Figure S20. SAOS data for PE-v. (A) Unshifted $\tan \delta$ and (D) the van Gurp-Palmen plot.

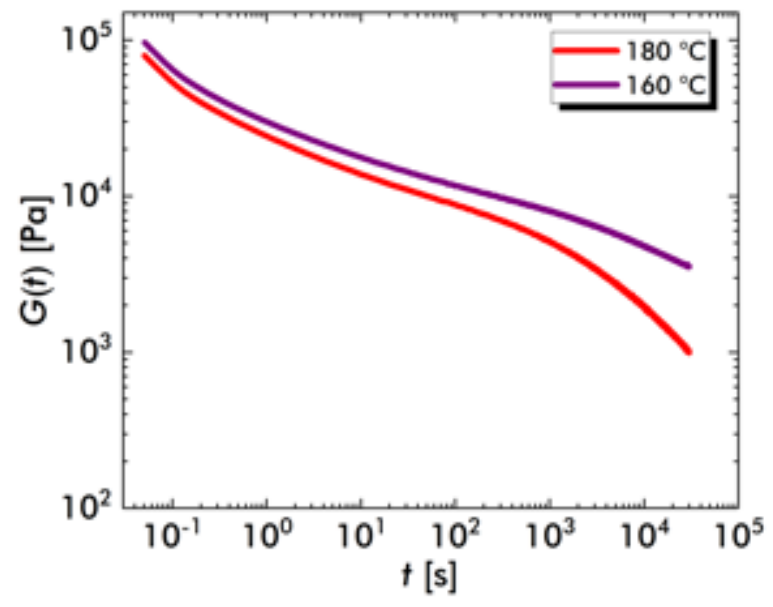

Figure S21. $8 \mathrm{~h}$ stress relaxations of PE- $v$ at 180 and $160{ }^{\circ} \mathrm{C}$. 


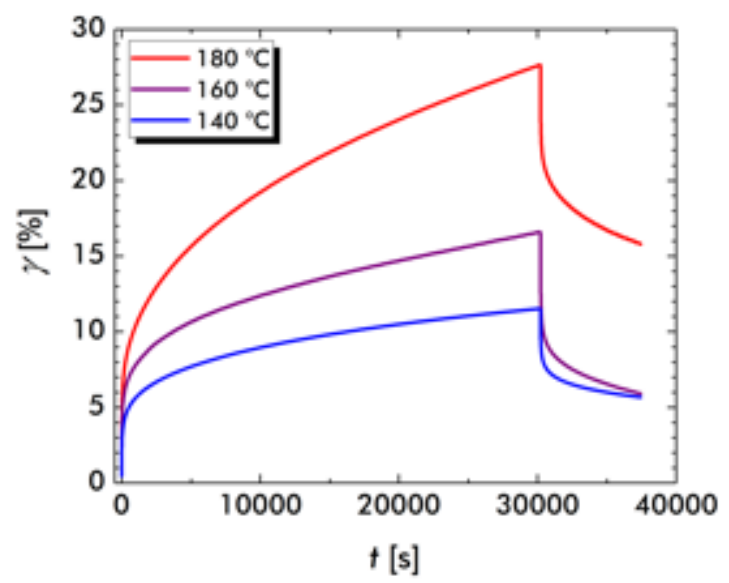

Figure S22. Raw strain measurements during creep and recovery of PE- $v . \sigma=500 \mathrm{~Pa}$ for all temperatures.

$P E$-v-sol. Figures S23A and S23B feature the unshifted dynamic moduli and $\tan \delta$. The data in the van Gurp-Palmen plot did not overlap (Figure S23C), indicating that PE-v-sol does not obey tTs. Figure S24 contains stress relaxation data. Figure S25 portrays the raw strain values measured during creep and recovery measurements. Figures S26A and S26B are the linear regressions utilized for calculating $J_{e}^{0}$ and $\eta_{0}$. Figure S27 features $J_{e}^{0}, \eta_{0}$, and $\tau$. 

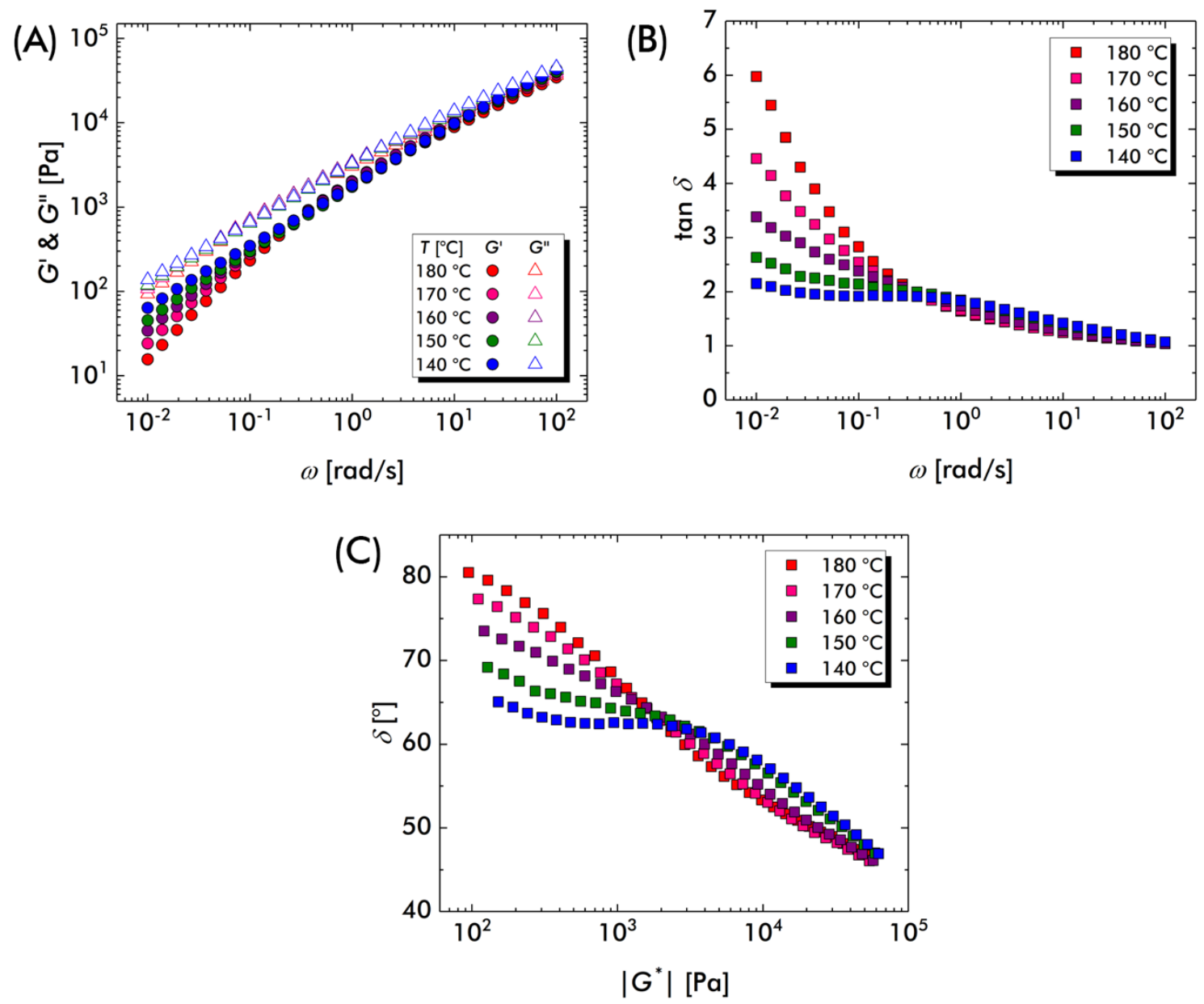

Figure S23. SAOS data for PE-v-sol. (A) $G^{\prime}$ and $G^{\prime \prime}$, (B) unshifted $\tan \delta$, (C) and the van GurpPalmen plot. 


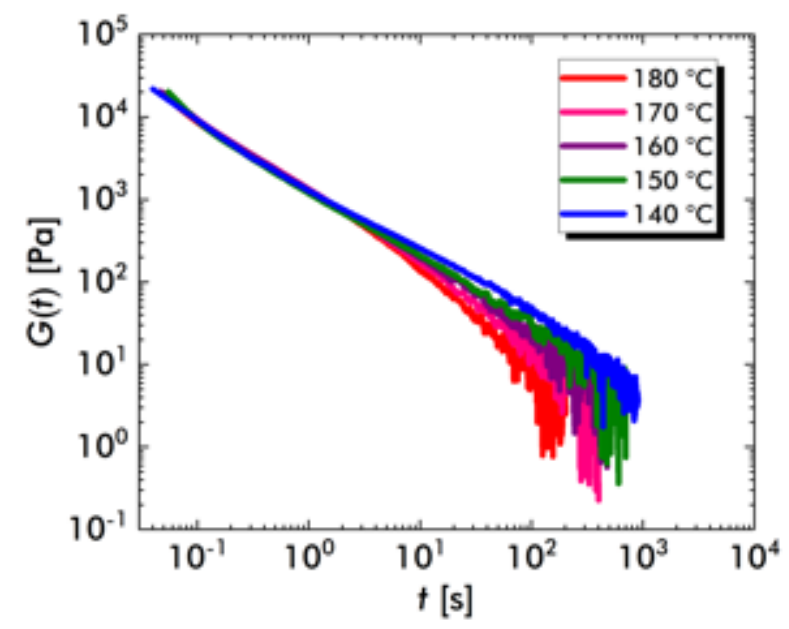

Figure S24. Stress relaxation of PE-v-sol.

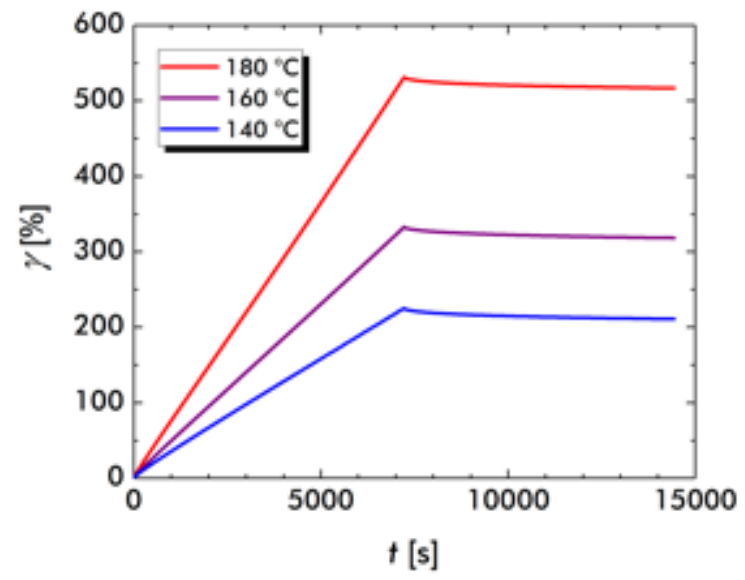

Figure S25. Raw strain measurements during creep and recovery of PE- $v$-sol. $\sigma=10$ Pa for all temperatures. 

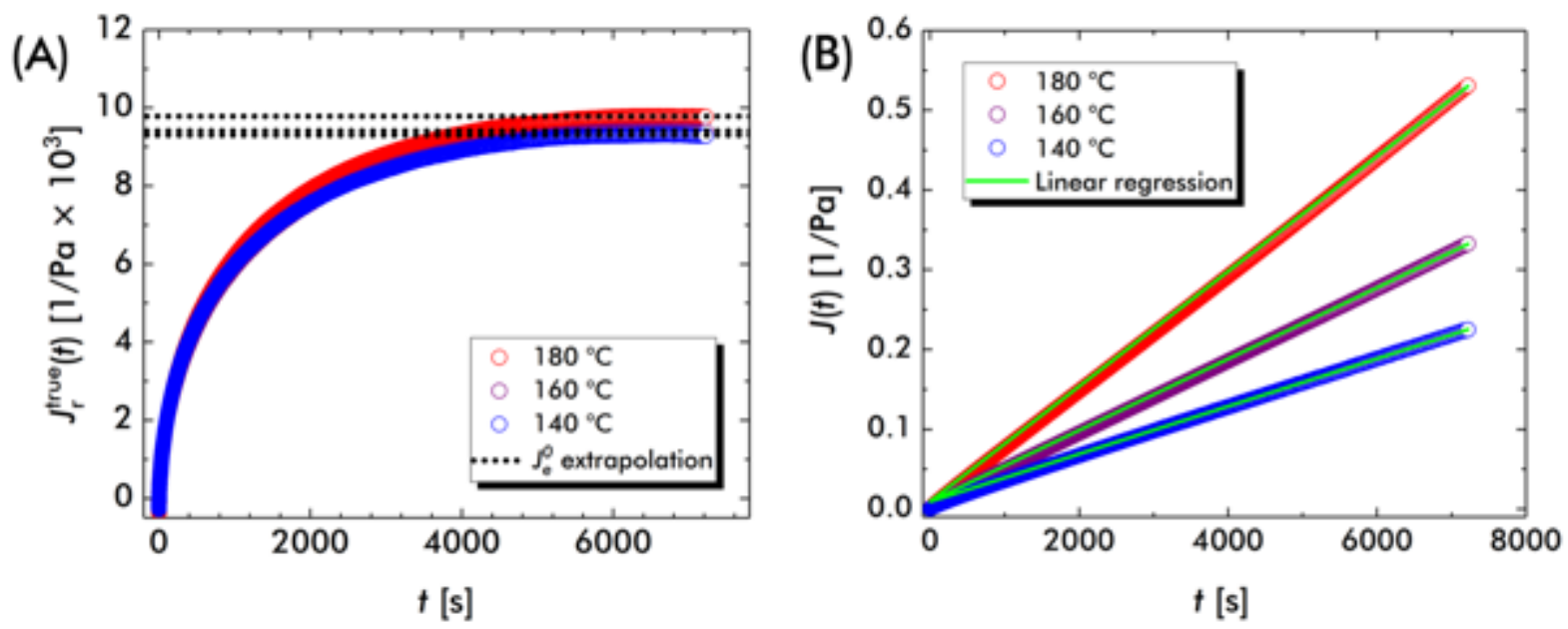

Figure S26. Creep compliance and recovery data for PE-v-sol at varying temperature. (A) Creep recovery. Dotted lines represent the plateau extrapolation used to calculate $J_{e}^{0}$. (B) Creep compliance. Green lines signify the linear extrapolations used to estimate $\eta_{0}$. 

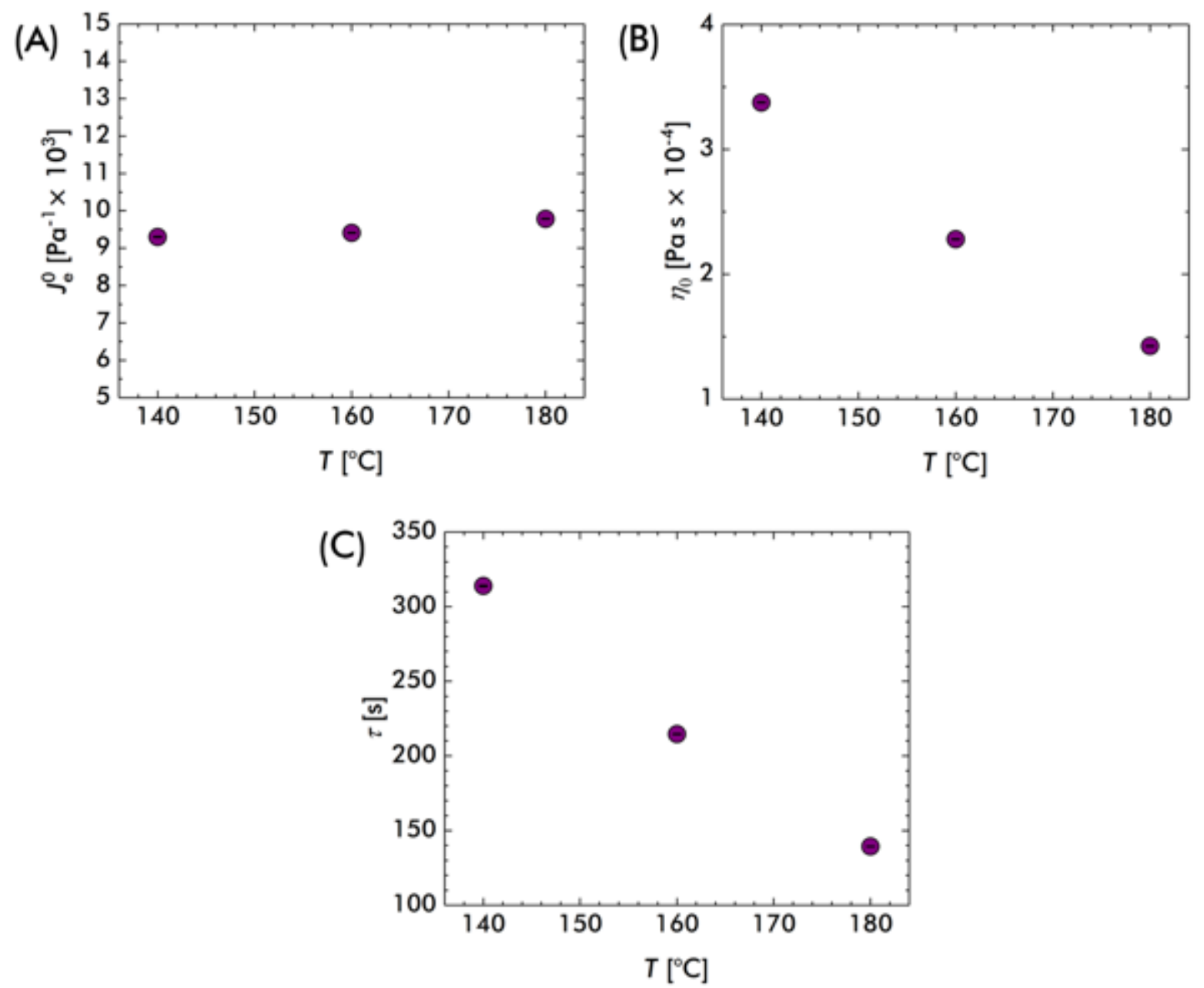

Figure S27. PE- $v$-sol macroscopic flow properties as determined by creep compliance and recovery measurements. (A) $J_{e}^{0},(\mathrm{~B}) \eta_{0}$, and (C) $\tau$.

$P E$-v-insol. Unshifted $\tan \delta$ data are displayed in Figure S28A. The lack of data overlap in the van Gurp-Palmen plot (Figure S28B) indicates that tTs fails for PE- $v$-insol. Figure S29 features stress relaxation data. Figure S30 displays the raw strain values measured during creep and recovery measurements. 

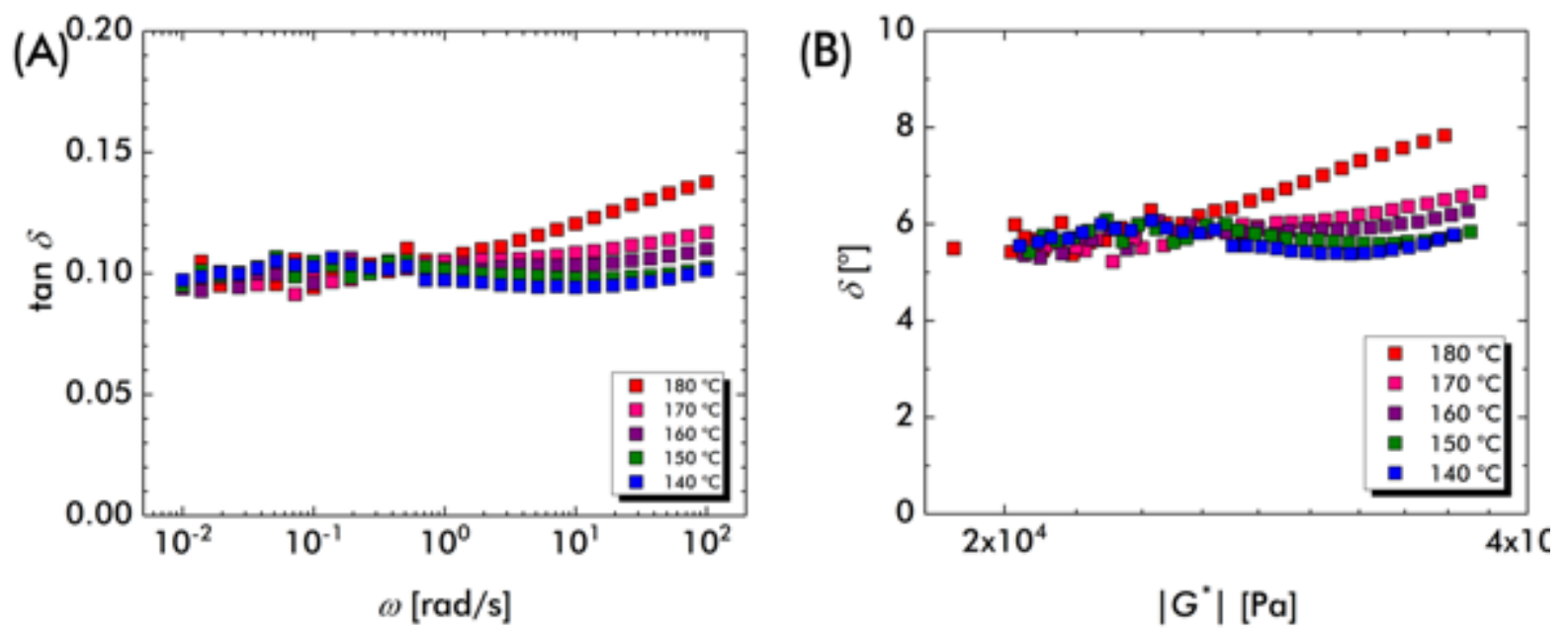

Figure S28. SAOS data for PE-v-insol. (A) Unshifted $\tan \delta$ and (D) the van Gurp-Palmen plot.

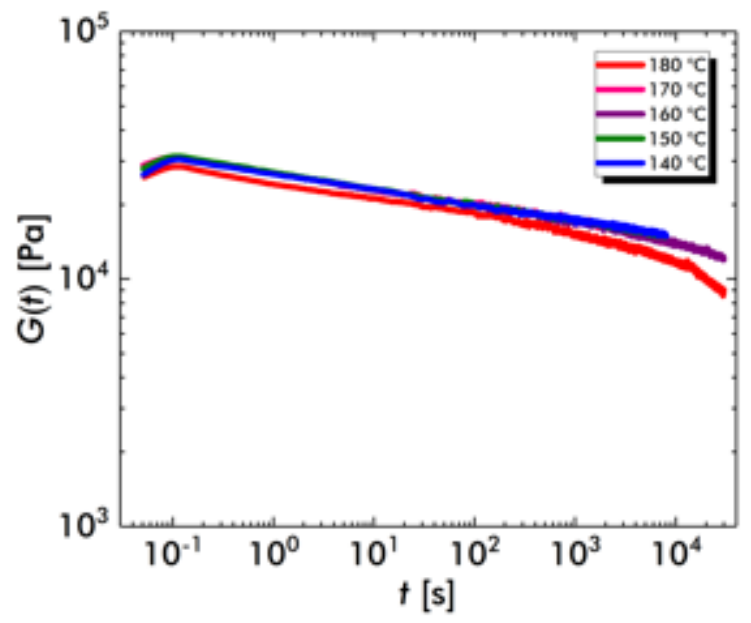

Figure S29. Stress relaxation of PE-v-insol. 


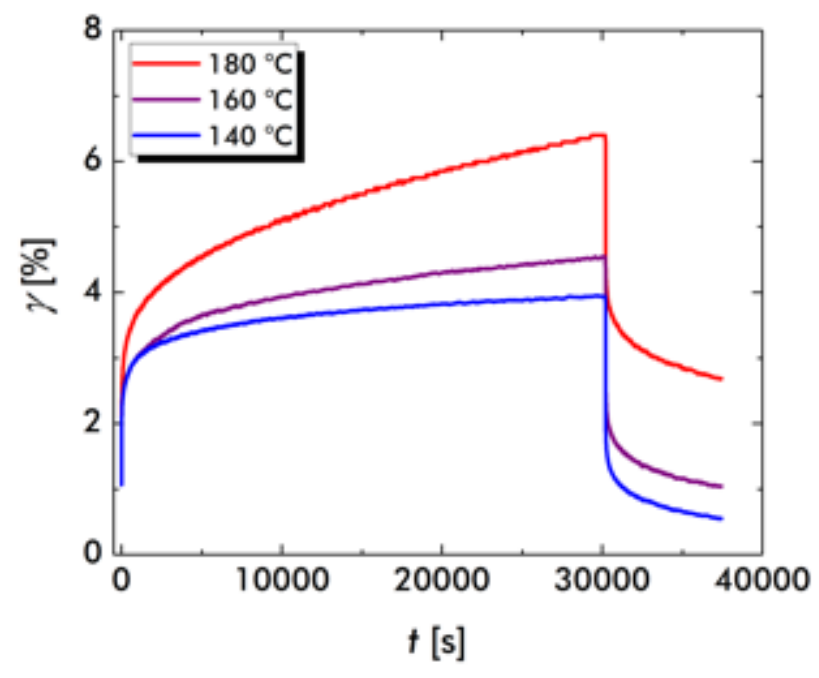

Figure S30. Raw strain measurements during creep and recovery of PE-v-insol. $\sigma=500 \mathrm{~Pa}$ for all temperatures.

Blend PE/PE-v-insol. Unshifted dynamic moduli and $\tan \delta$ are shown in Figures S31A and S31B. The lack of data overlap in the van Gurp-Palmen plot (Figure S31C) indicates that tTs fails for blend PE/PE- $v$-insol. Figure S32 features stress relaxation data. Figure S33A displays the raw strain values recorded during creep and recovery measurements. Figure S33B shows the $J(t)$ data. At all temperatures, blend PE/PE-v-insol does not achieve the steady-state flow scaling of 1. 

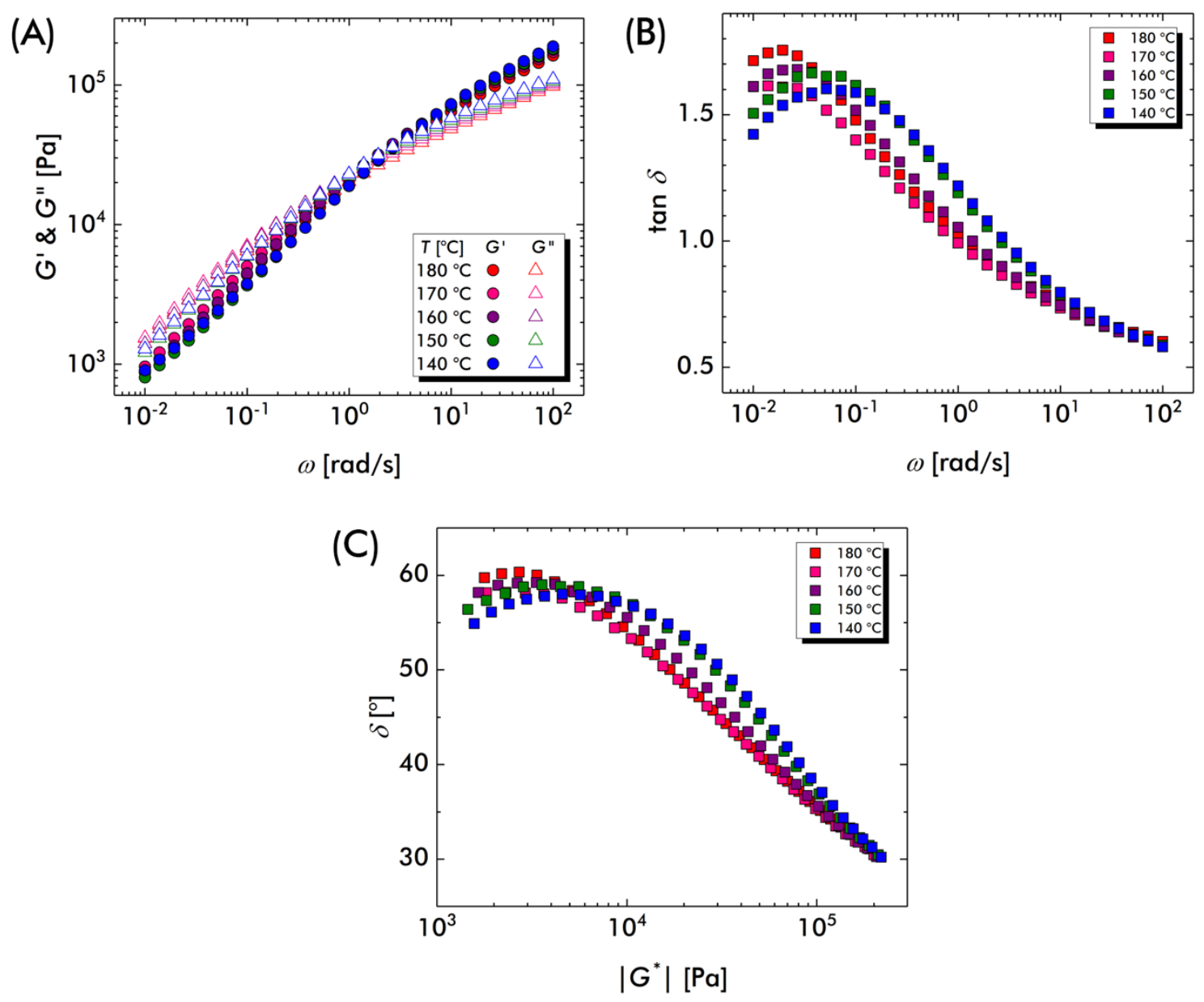

Figure S31. SAOS data for blend PE/PE-v-insol. (A) $G^{\prime}$ and $G^{\prime \prime},(\mathrm{B})$ unshifted $\tan \delta$, (C) and the van Gurp-Palmen plot. 


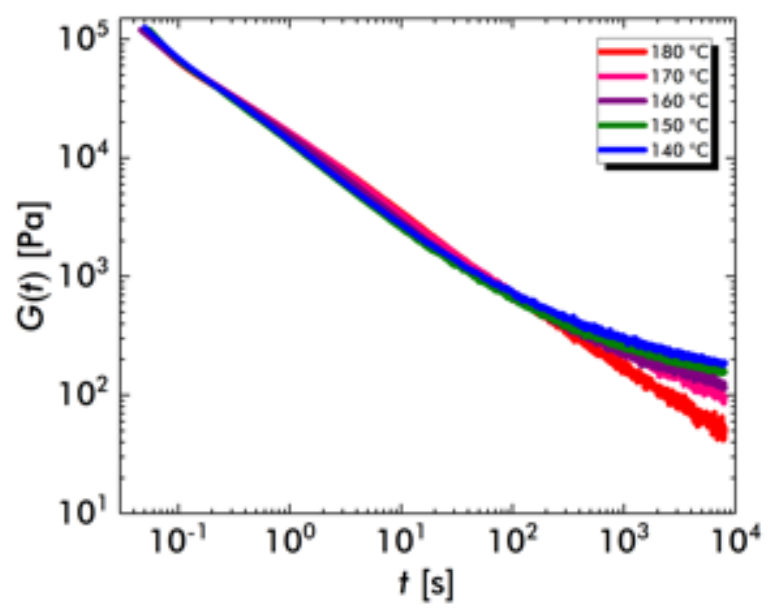

Figure S32. Stress relaxation of blend PE/PE- $v$ insol.
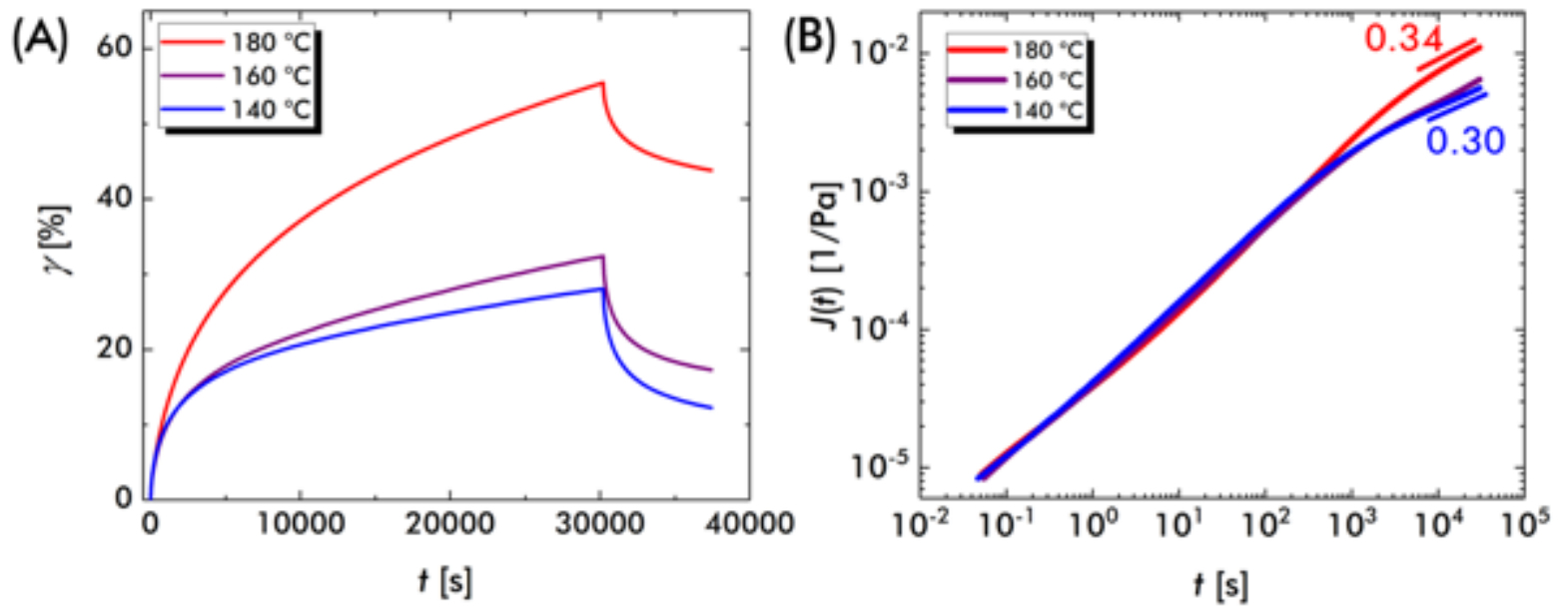

Figure S33. (A) Raw strain measurements during creep and recovery of blend PE/PE- $v$-insol. $\sigma=50 \mathrm{~Pa}$ for all temperatures. (B) Creep compliance data for blend PE/PE-v-insol.

Blend PE-v-sol/PE-v-insol. Unshifted dynamic moduli and $\tan \delta$ are shown in Figures S34A and S34B. The lack of data overlap in the van Gurp-Palmen plot (Figure S34C) indicates that tTs fails for blend PE-v-sol/PE-v-insol. Figure S35 features stress relaxation data. Figure S36A features the raw strain values recorded during creep and recovery measurements. Figure S36B shows the $J(t)$ 
data. At all temperatures, blend PE-v-sol/PE-v-insol does not achieve the steady-state flow scaling of 1 .
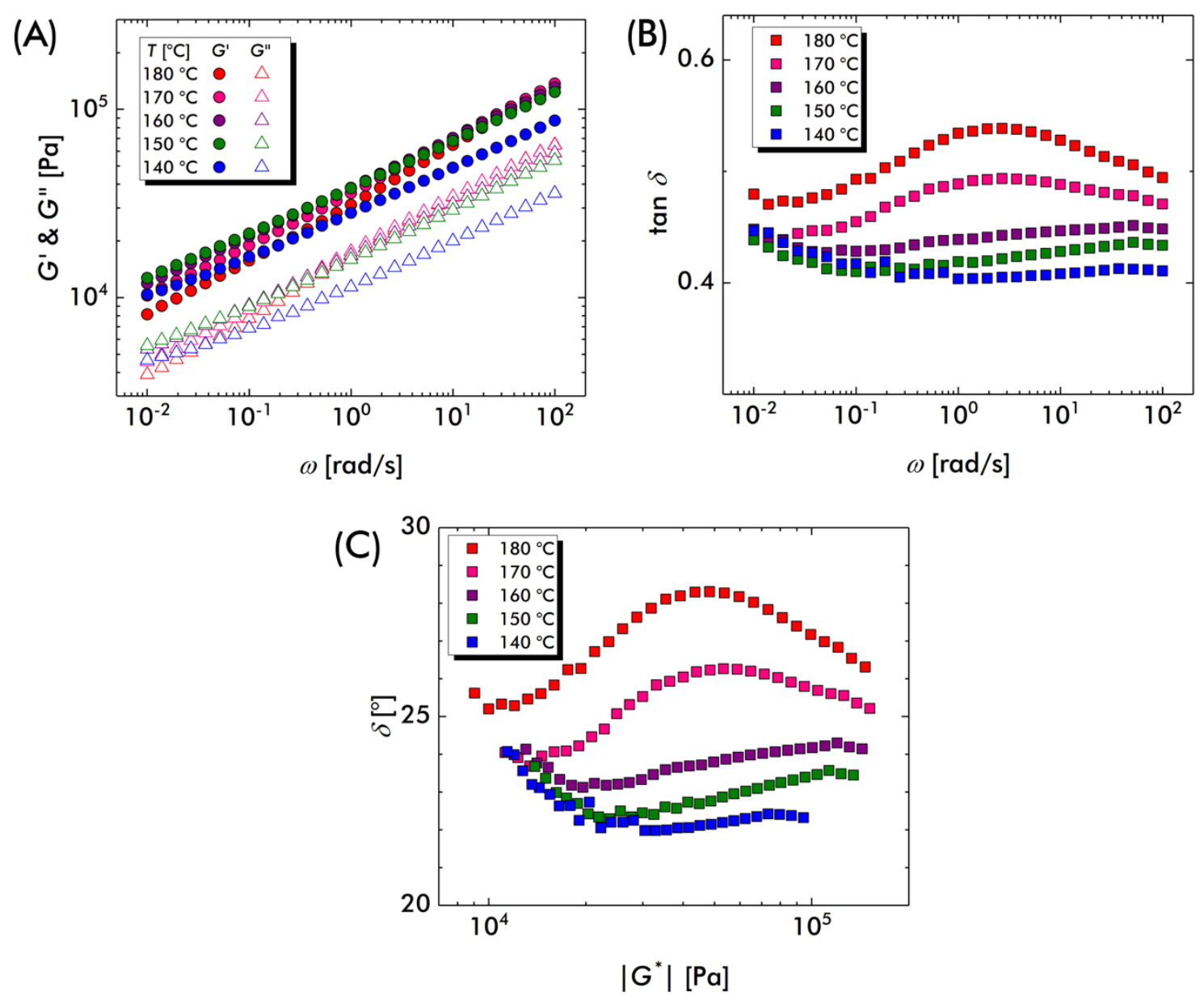

Figure S34. SAOS data for blend PE- $v$-sol/PE- $v$-insol. (A) $G^{\prime}$ and $G^{\prime \prime}$, (B) unshifted $\tan \delta$, (C) and the van Gurp-Palmen plot. 


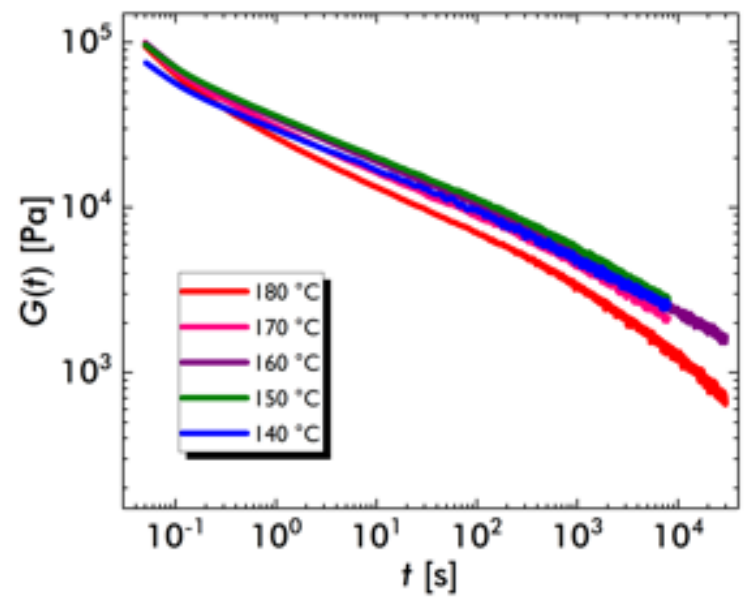

Figure S35. Stress relaxation of blend PE- $v$ sol/PE-v-insol.
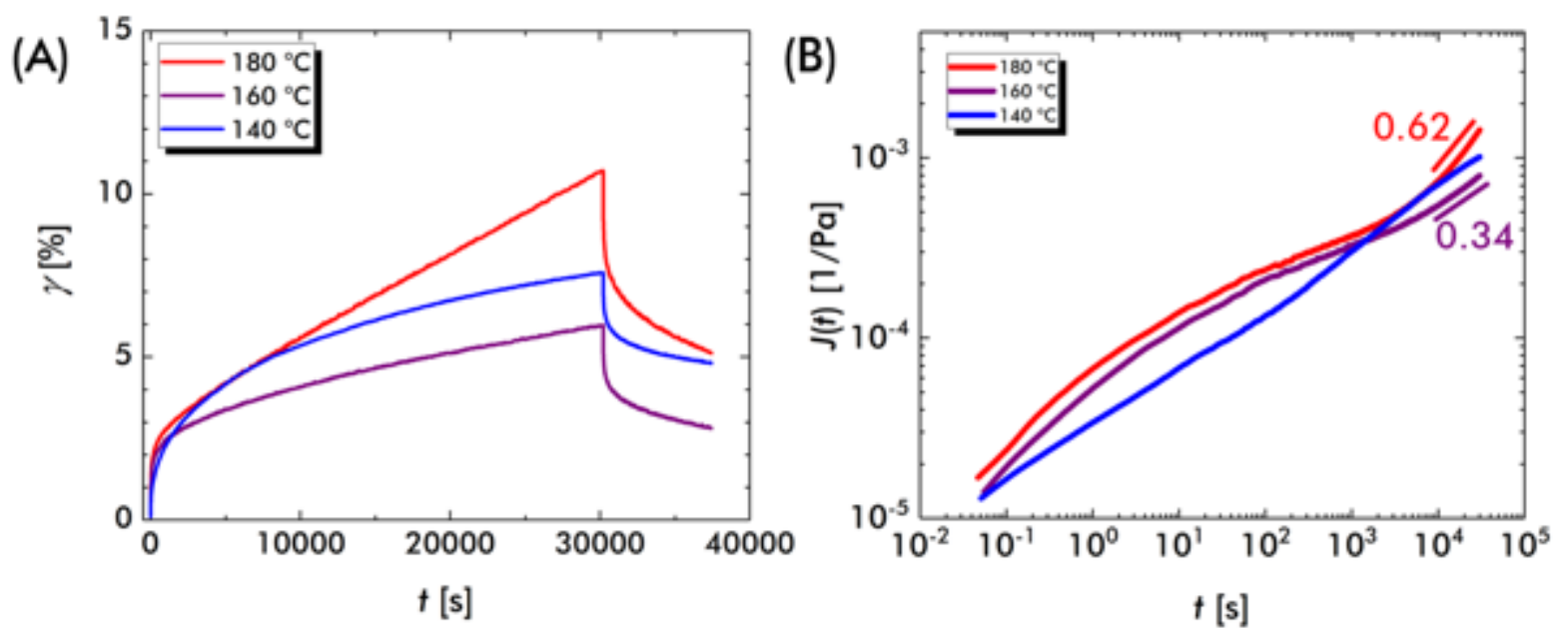

Figure S36. (A) Raw strain measurements during creep and recovery of blend PE- $v$-sol/PE- $v$ insol. $\sigma=75 \mathrm{~Pa}$ for all temperatures. (B) Creep compliance data for blend PE- $v$-sol/PE- $v$-insol.

\section{Influence of thermal degradation on PE-g and blend PE-v-sol/PE-v-insol}

Figure S37 compares creep compliance data of PE-g and blend PE- $v$-sol/PE- $v$-insol that were measured using different temperature protocols. In the ascending scheme, creep and recovery measurements were performed at (I) 140 , (II) 160 , and (III) $180^{\circ} \mathrm{C}$. Using this scheme, the samples 
creep faster as the temperature is increased. In the descending scheme, measurements were performed at (I) $180^{\circ} \mathrm{C}$, (II) $160^{\circ} \mathrm{C}$, and (III) $140{ }^{\circ} \mathrm{C}$. Under this scheme, the samples creep faster as the temperature is decreased. We attribute this unexpected behavior in the descending scheme to degradation of the dioxaborolane maleimide grafts. During the creep and recovery measurements, the sample is held at each temperature for approximately $11 \mathrm{~h}$. At $180^{\circ} \mathrm{C}$, the grafts are potentially susceptible to deboronation or oxidation. ${ }^{\mathrm{S} 2}$ In contrast, PE- $v$, PE-v-insol, and blend PE/PE-v-insol did not seem to degrade at the elevated temperatures. 

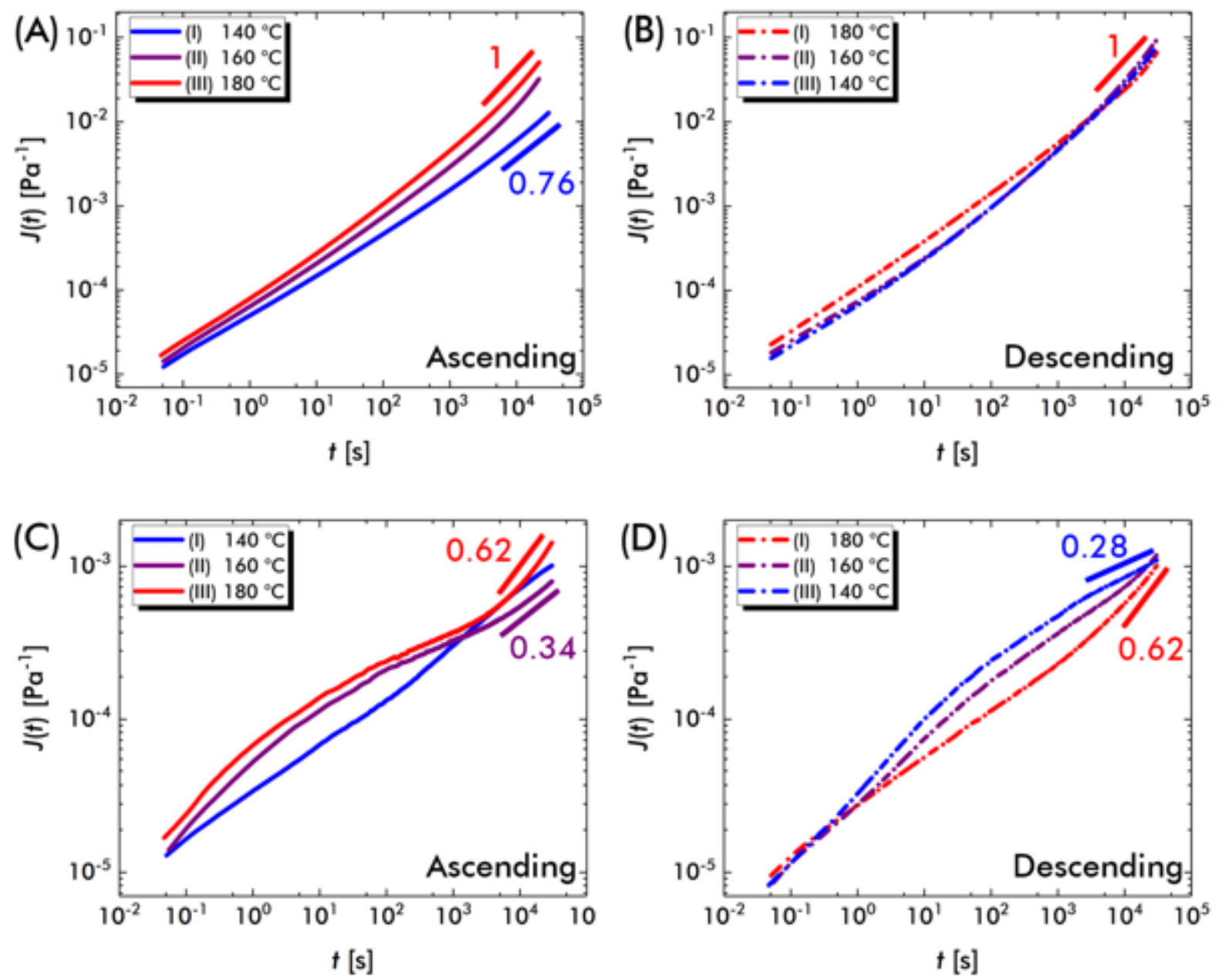

Figure S37. Comparison of creep compliance data collected in an ascending or descending temperature order. (A \& B) PE-g and (C \& D) blend PE- $v$-sol/PE- $v$-insol.

\section{Comparison of PE, PE-g, PE-v, PE-v-sol, and PE-v-insol rheology}

Figure S38 compares the dynamic moduli of PE, PE-g, PE- $v$, PE- $v$-sol, and PE- $v$-insol. Figure S39 compares the stress relaxation and creep compliance of PE and PE-v-sol. 

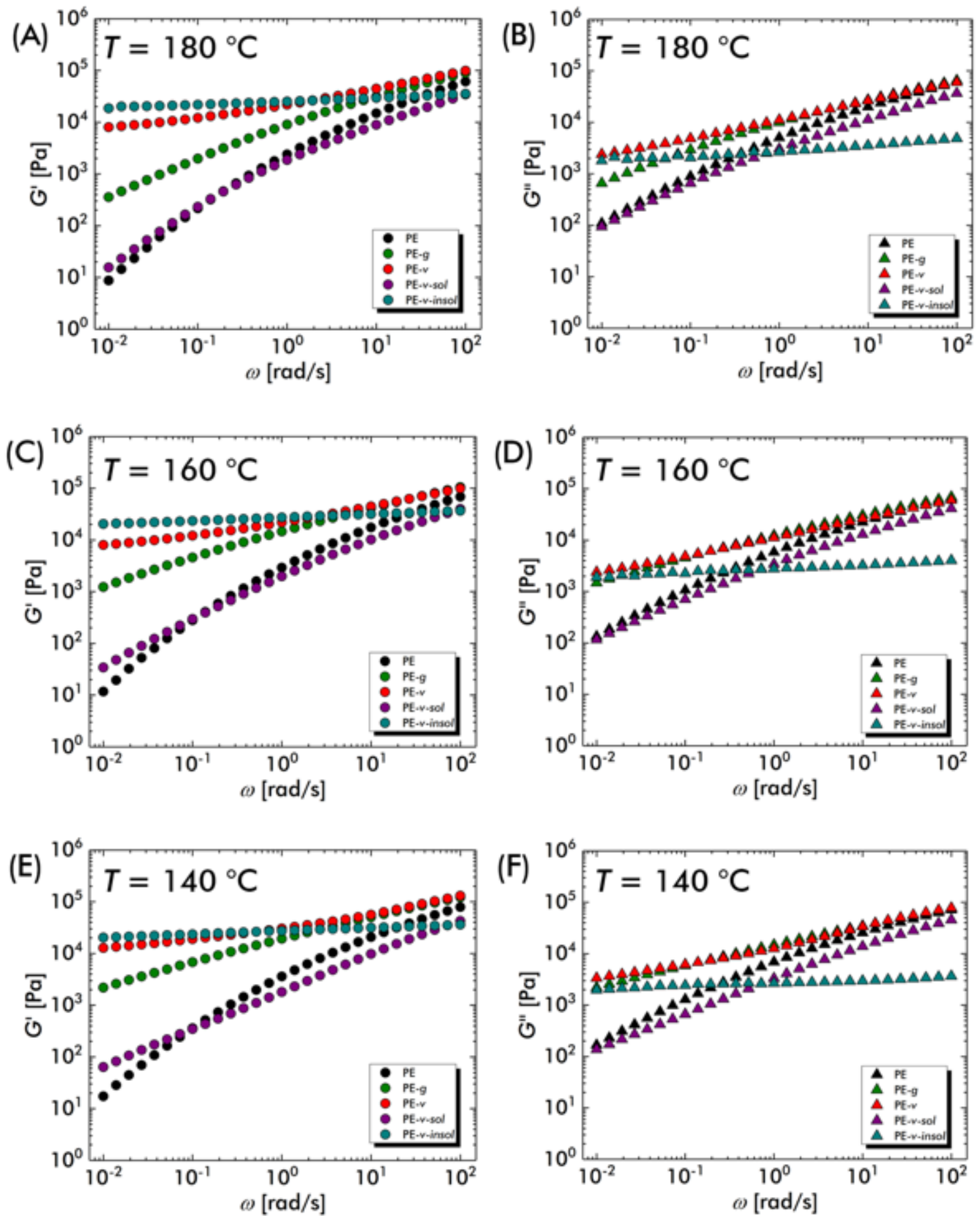

Figure S38. Comparison of $G^{\prime}$ and $G^{\prime \prime}$ for PE, PE-g, PE- $v$, PE- $v$-sol, and PE- $v$-insol at (A \& B) $180,(\mathrm{C} \& \mathrm{D}) 160$, and $(\mathrm{E} \& \mathrm{~F}) 140^{\circ} \mathrm{C}$. 

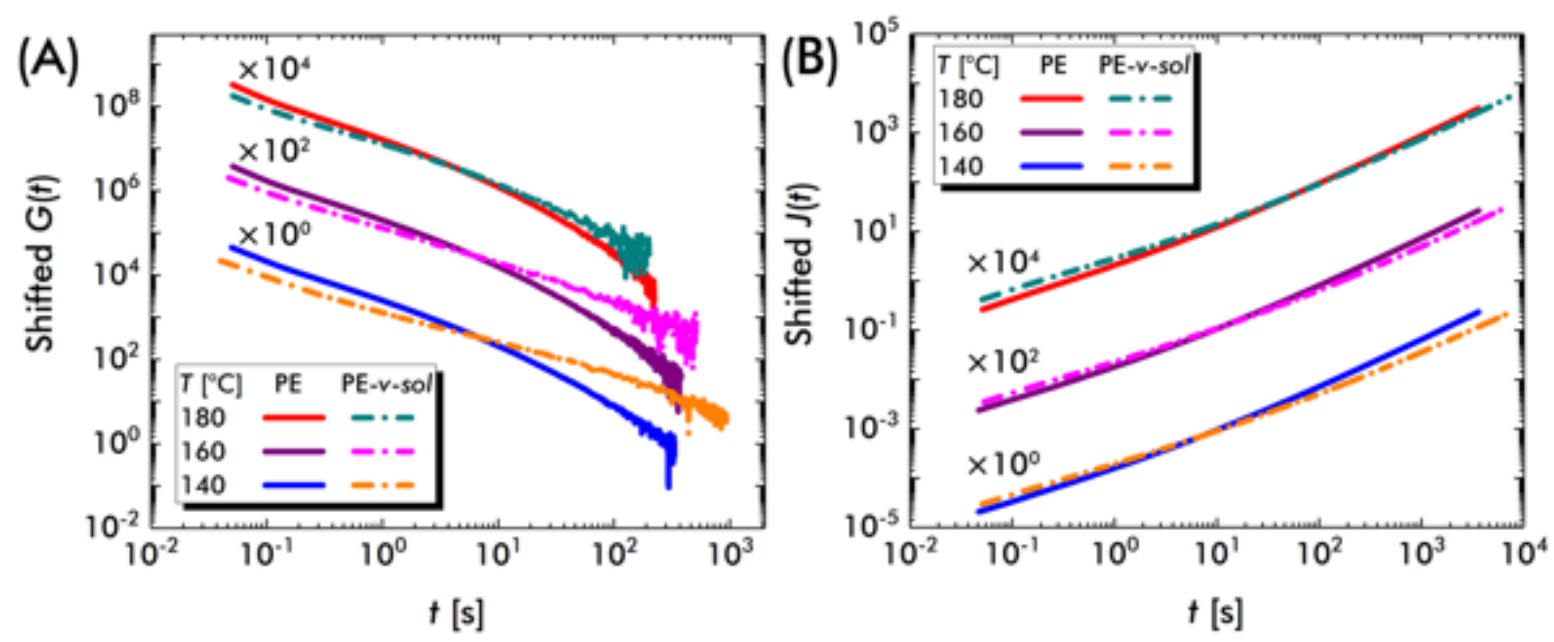

Figure S39. Comparison of (A) stress relaxation and (B) creep compliance for PE and PE- $v$-sol. Data are shifted for clarity.

\section{Comparison of PE-v and blend PE-v-sol/PE-v-insol rheology}

Figure S40 compares the dynamic moduli of PE- $v$ and blend PE- $v$-sol/PE- $v$-insol, while Figure S41 compares the stress relaxation and creep compliance for those samples.
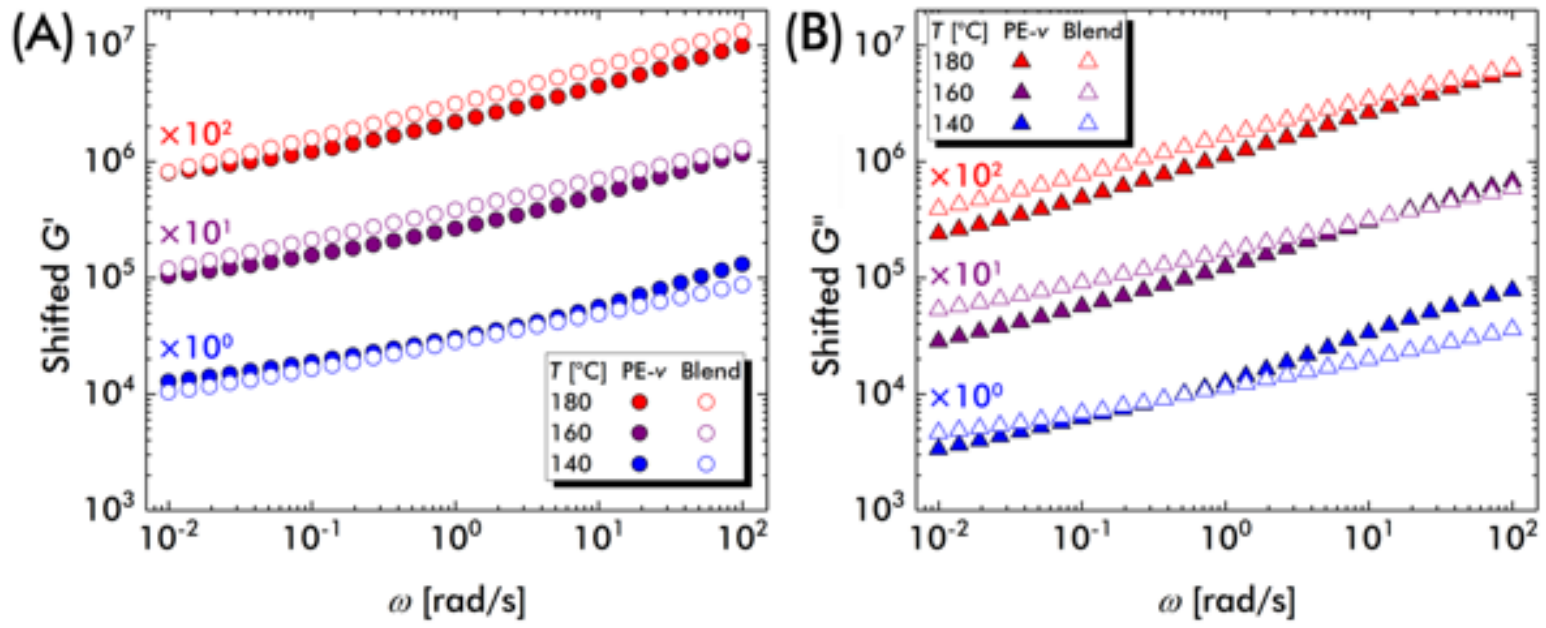

Figure S40. Comparison of (A) $G$ ' and (B) $G$ ' moduli for PE- $v$ and blend PE- $v$-sol/PE- $v$-insol. Data are shifted for clarity. 

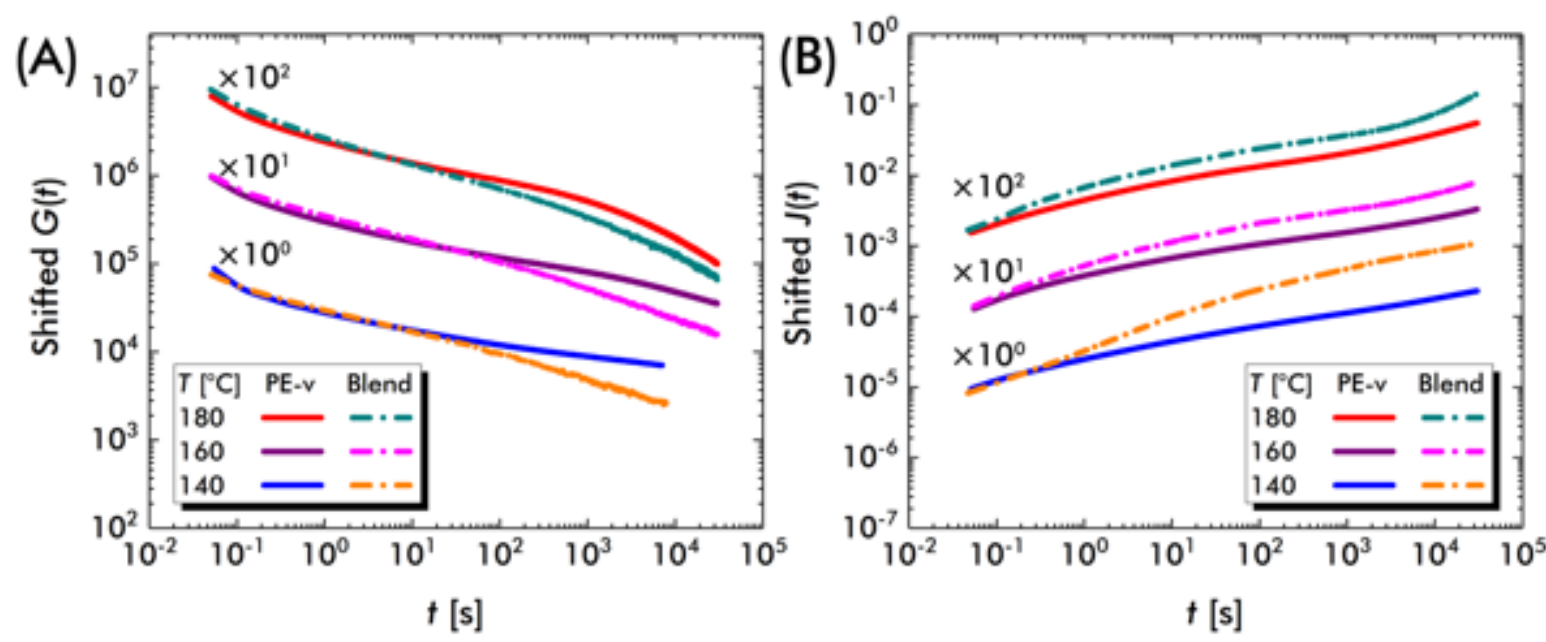

Figure S41. Comparison of (A) stress relaxation and (B) creep compliance for PE- $v$ and blend PE-v-sol/PE-v-insol. Data are shifted for clarity.

\section{Conversion of creep compliance to dynamic moduli}

Creep compliance data can be converted into the dynamic moduli using the following relationships: ${ }^{\mathrm{S} 3, \mathrm{~S} 4}$

$$
\begin{aligned}
& J^{\prime}(\omega)=J(t)+0.0007[J(32 t)-J(16 t)]-0.0185[J(16 t)-J(8 t)] \\
& +0.197[J(8 t)-J(4 t)]-0.778[J(4 t)-J(2 t)] \\
& -0.181[J(t)-J(t / 2)]-0.049[J(t / 4)-J(t / 8)] \\
& J^{\prime \prime}(\omega)=-0.470[J(4 t)-J(2 t)]+1.674[J(2 t)-J(t)]+0.198[J(t)-J(t / 2)] \\
& +0.620[J(t / 2)-J(t / 4)]+0.012[J(t / 4)-J(t / 8)] \\
& +0.172[J(t / 8)-J(t / 16)]+0.043[J(t / 32)-J(t / 64)] \\
& +0.012[J(t / 128)-J(t / 256)] \\
& G^{\prime}(\omega)=\frac{J^{\prime}(\omega)}{\left[J^{\prime}(\omega)\right]^{2}+\left[J^{\prime \prime}(\omega)\right]^{2}}
\end{aligned}
$$




$$
\begin{gathered}
G^{\prime \prime}(\omega)=\frac{J^{\prime \prime}(\omega)}{\left[J^{\prime}(\omega)\right]^{2}+\left[J^{\prime \prime}(\omega)\right]^{2}} \\
\omega=1 / t
\end{gathered}
$$

Figure S43 compares the PE- $v$ dynamic moduli measured by SAOS and the values estimated using the creep data from Figure 6C. By transforming the creep data, the minimum $\omega$ of the dynamic moduli was lowered to $10^{-3} \mathrm{rad} / \mathrm{s}$.

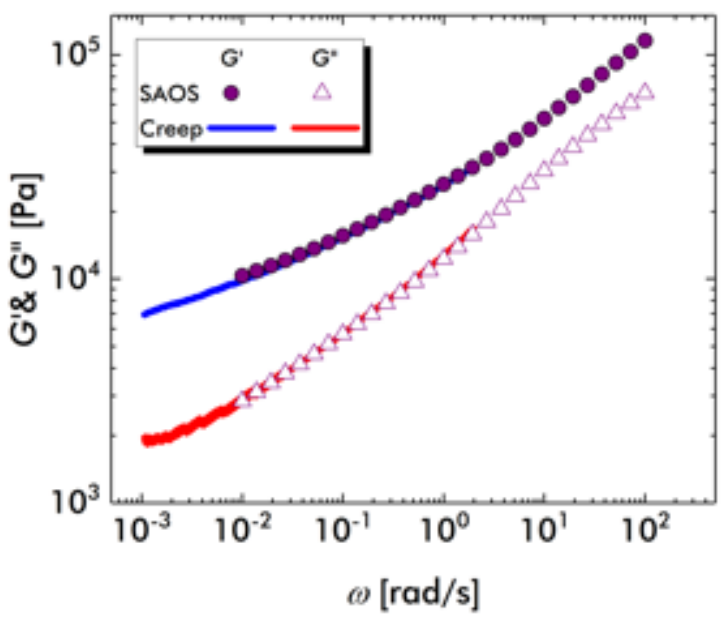

Figure S43. Dynamic moduli of PE- $v$ at $160^{\circ} \mathrm{C}$. The blue and red lines were calculated by transforming the data from Figure 6C using eqns. S1-S5.

\section{Estimation of cross-link densities}

For PE vitrimers, the cross-link density estimated from FTIR $\left(v_{F T I R}\right)$ can be calculated using the formula 


$$
v_{F T I R}=\frac{C_{\text {cross-link }}}{C_{P E}} \frac{\rho_{P E} N_{a v}}{M_{P E}}
$$

where $\frac{C_{\text {cross-link }}}{C_{P E}}$ is the molar ratio of the bis-dioxaborolane cross-linker to the ethylene repeat unit, $\rho_{P E}$ is the density of amorphous PE $\left(0.853 \mathrm{~g} / \mathrm{cm}^{3}\right),{ }^{\mathrm{S} 5} M_{P E}$ is the molecular weight of the ethylene repeat unit, and $N_{a v}$ is Avogadro's constant. The effective cross-link density $\left(v_{e f f}\right)$ can be estimated using

$$
v_{e f f}=\frac{G^{*} N_{a v}}{R T}
$$

where $G^{*}$ is the complex plateau modulus, $T$ is temperature, $R$ is the gas constant, and $N_{a v}$ is Avogadro's constant. Table S1 compares the $v_{F T I R}$ and $v_{\text {eff }}$ values for PE-v-insol.

Table S1. Comparison of $v_{F T I R}$ and $v_{e f f}$ for PE vitrimer materials.

\begin{tabular}{lcrrr}
\hline $\begin{array}{l}\text { Sample } \\
\text { name }\end{array}$ & $\begin{array}{r}\text { [Cross-linker }] /[\text { Ethylene }] \\
\times \mathbf{1 0}^{\mathbf{3}}\end{array}$ & $\begin{array}{r}\boldsymbol{G}^{*} \\
{\left[\mathbf{P a} \times \mathbf{1 0}^{-4}\right]}\end{array}$ & $\begin{array}{r}\boldsymbol{v}_{\text {FTIR }} \\
{\left[\mathbf{n m}^{-3} \times \mathbf{1 0}^{2}\right]}\end{array}$ & $\begin{array}{r}\boldsymbol{v}_{\text {eff }} \\
{\left[\mathbf{n m}^{-3}\right]}\end{array}$ \\
\hline PE-v-insol & 1.3 & 2.8 & 4.7 & 0.47 \\
\hline
\end{tabular}

\section{References}

${ }^{\text {S1} V a n ~ G u r p, ~ M . ; ~ P a l m e n, ~ J . ~ R h e o l . ~ B u l l . ~ 1998, ~ 67, ~ 5-8 . ~}$

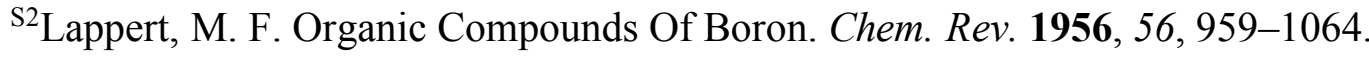

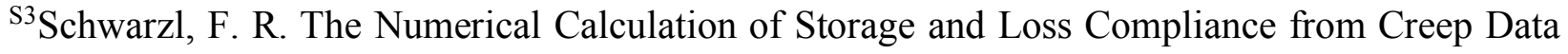
for Linear Viscoelastic Materials. Rheol. Acta 1969, 8, 6-17. 


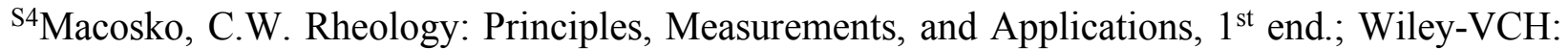
New York, NY, 1994.

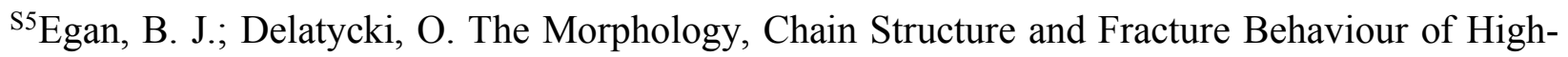
Density Polyethylene. J. Mater. Sci. 1995, 30, 3307-3318. 